\title{
Leg Scanner for Detection and Tracking of Evolving Pigmented Skin Lesions for Melanoma Cancer Prevention
}

\author{
By \\ Alisa Bondarev \\ A thesis submitted to \\ the Faculty of Graduate Studies and Research \\ in partial fulfilment of \\ the requirements for the degree of \\ M.A.Sc. Biomedical Engineering \\ Ottawa-Carleton Institute for Computer Science \\ School of Computer Science \\ Carleton University \\ Ottawa, Ontario \\ May 10, 2019 \\ (C) Copyright \\ 2019, Alisa Bondarev
}


The undersigned hereby recommend to the Faculty of Graduate Studies and Research acceptance of the thesis,

Leg Scanner for Detection and Tracking of Evolving Pigmented Skin Lesions for Melanoma Cancer Prevention submitted by Alisa Bondarev

\begin{tabular}{c}
\hline Prof., Douglas Howe \\
(Director, School of Computer Science) \\
\hline $\begin{array}{c}\text { Prof., Doron Nussbaum } \\
\text { (Thesis Supervisor) }\end{array}$
\end{tabular}

Carleton University

May 10, 2019 


\section{Abstract}

Melanoma is an aggressive skin cancer prevalent among the Caucasian population. The site of melanoma is gender dependent, for women the prevalent site is the legs. Success of the treatment is strongly correlated with early dysplastic nevus excision. Diagnosis relies on full-body skin examination and baseline photography to locate evolving, and therefore suspicious, moles.

We designed the first semi-automated legs scanner which uses one camera and laser range finder to capture images and measurements respectively. The scanner circulates around the leg, along its full length. From the collected data, a $3 D$ leg model with moles is created. The moles are registered over temporal scans for comparison and detection of moles' evolution.

Our algorithm was tested on simulated leg model that changes over time. The average accuracy of mole registration from two temporal scans at optimal scanner settings was $94.87 \%$ with standard deviation 6.10 . 


\section{Acknowledgements}

I would like to sincerely thank my supervisor, Dr. Doron Nussbaum (from Department of Computer Science, Carleton University) for his unstinted continuous support and valuable guidance throughout the thesis. 
To my beloved parents, Anna and Alexander.

To my great husband, Yauhen, and our beautiful children, Anastasia and Nicole. To my supportive siblings, Artyom, Kirill, and Olga. 


\section{List of Abbreviations}

$\begin{array}{ll}\text { AI } & \text { Artificial Intelligence } \\ \text { CAD } & \text { Computer Aided Diagnosis } \\ \text { CPD } & \text { Coherent Point Drift } \\ \text { DOF } & \text { Depth of Field } \\ \text { EM } & \text { Expectation Maximization } \\ \text { GMM } & \text { Gaussian Mixture Models } \\ \text { HSV } & \text { Hue Saturation Value } \\ \text { LRF } & \text { Laser Range Finder } \\ \text { MM } & \text { Malignant Melanoma } \\ \text { PSL } & \text { Pigmented Skin Lesion } \\ \text { RANSAC } & \text { Random Sample Consensus } \\ \text { ROI } & \text { Region of Interest } \\ \text { SDG } & \text { Simulation Data Generator } \\ \text { SIFT } & \text { Scale-Invariant Feature Transformation } \\ \text { TBSE } & \text { Total Body Skin Examination } \\ \text { TBSI } & \text { Total Body Skin Imagining } \\ \text { TBSS } & \text { Total Body Scanning System } \\ \text { UVR } & \text { Ultraviolet Radiation } \\ \text { WBP } & \text { Whole Body Photography }\end{array}$




\section{Contents}

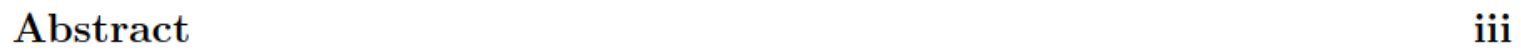

Acknowledgements iv

List of Abbreviations vi

1 Introduction 1

1.1 Problem definition $\ldots \ldots \ldots \ldots \ldots$

1.2 Contribution summary $\ldots \ldots \ldots \ldots \ldots \ldots$

1.3 Thesis organization $\ldots \ldots \ldots \ldots \ldots \ldots \ldots \ldots$

$\begin{array}{lll}2 & \text { Background } & 6\end{array}$

2.1 The Human Skin Structure. . . . . . . . . . . . . . . . . . . . . . . 7

2.2 Malignant Melanoma . . . . . . . . . . . . . . . . . . . . . . . . 9

2.3 Diagnosis of Melanoma . . . . . . . . . . . . . . . . . . . . . 11

2.3 .1 Naked eye - ABCDE rule . . . . . . . . . . . . . . 13

2.3 .2 7-point checklist. . . . . . . . . . . . . . . . . . . 14

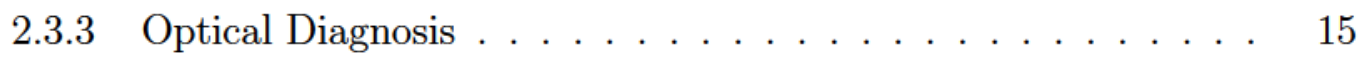

2.3 .3 .1 Dermoscopy . . . . . . . . . . . . . . . . . . . 15

2.3.3.2 Transillumination technique - Nevoscope . . . . . . . 15

$2.3 .3 .3 \quad$ Baseline Imaging . . . . . . . . . . . . . . . . 16

2.3 .3 .4 Total Cutaneous Photography . . . . . . . . . . . 16

$2.3 .4 \quad$ Computer Aided Diagnosis . . . . . . . . . . . . . . . . . 18

2.3 .5 Automated Multiple Lesion Diagnosis . . . . . . . . . . . 20 
$2.3 .5 .1 \quad$ Image Storage Systems . . . . . . . . . . . . . 22

2.3 .5 .2 Mole Localization. . . . . . . . . . . . . . . 22

2.3 .5 .3 Mole Registration . . . . . . . . . . . . . . 23

2.3.5.4 Mole Localization and Registration . . . . . . . . 25

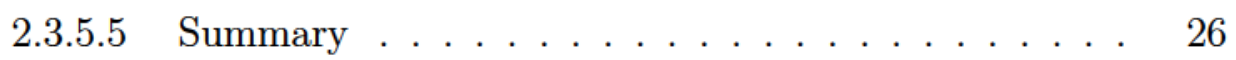

2.4 Computer Vision $\ldots \ldots \ldots \ldots \ldots \ldots$

2.4 .1 Pinhole Camera Model . . . . . . . . . . . . . . . . . . 27

$2.4 .2 \quad$ Epipolar Geometry . . . . . . . . . . . . . . . . . . . . . 29

$2.4 .3 \quad 3 D$ Registration Algorithm . . . . . . . . . . . . . . . . 32

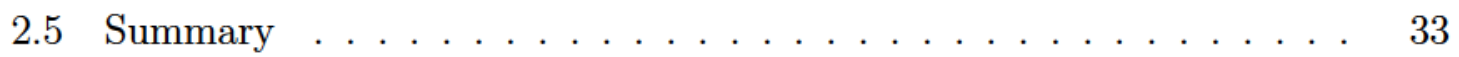

3 Lower Limbs Scanner Hardware Design 35

3.1 Hardware design $\ldots \ldots \ldots \ldots \ldots$

3.1 .1 The imaging subsystem $\ldots \ldots \ldots \ldots$

3.1.1.1 Illumination . . . . . . . . . . . . . . . . . . 42

3.1 .1 .2 Background . . . . . . . . . . . . . . . . . . 42

3.1 .2 The distance measurement subsystem . . . . . . . . . . . 43

3.1 .3 Vertical rail track subsystem . . . . . . . . . . . . . . . . 44

3.2 The Scanning Procedure $\ldots \ldots \ldots$

$3.2 .1 \quad$ Definition of Coordinate Systems . . . . . . . . . . 47

3.3 System Limitations $\ldots \ldots \ldots \ldots$

3.4 Conclusions $\ldots \ldots \ldots \ldots \ldots$

\begin{tabular}{lll}
\hline & Methodology & 52
\end{tabular}

4.1 Intra-exploration Mole Mapping . . . . . . . . . . . . . . . . . 53

4.1 .1 Mole Location . . . . . . . . . . . . . . . . . . . . . . . 57

4.1 .1 .1 Region of Mole Distortion . . . . . . . . . . . 61

4.1 .2 Mole Pairs . . . . . . . . . . . . . . . . . . . 65

4.1 .3 Mole Sets . . . . . . . . . . . . . . . . . . . 66

4.1.3.1 Limitations of Mole Sets Creations . . . . . . . . . 66

$4.1 .4 \quad 3 D$ Mole Map . . . . . . . . . . . . . . . . . . . . . . 67

4.2 Temporal Scan Operations $\ldots \ldots \ldots$ 
$4.2 .1 \quad$ Match by CPD Algorithm . . . . . . . . . . . . . 71

4.3 Limitations of Temporal Scan Operations . . . . . . . . . . . . 73

$4.3 .1 \quad$ Error Carry Over from Mole Sets Creation . . . . . . . . . . 74

4.3 .2 Error in Generation of Correspondence Map . . . . . . . . 75

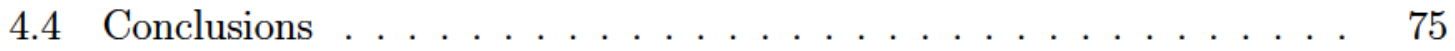

\begin{tabular}{|lll}
5 & Simulation Design, Results and Discussion & 77
\end{tabular}

5.1 Simulation Data Generator . . . . . . . . . . . . . . . . . . . . . . 78

$5.1 .1 \quad$ Leg's Shape $\ldots \ldots \ldots \ldots$. . . . . . . . . . . . . . . . . . . 78

5.1 .2 Moles . . . . . . . . . . . . . . . . . . . . . . . . . 79

$5.1 .3 \quad$ LRF data collection . . . . . . . . . . . . . . . . . . . . 81

5.1 .4 Camera Data Collection . . . . . . . . . . . . . . . . . 82

5.1.4.1 Camera's Transformation and Orientation . . . . . . 82

5.1 .5 Simulation of Temporal Scan . . . . . . . . . . . . 84

5.2 Scenarios Generator . . . . . . . . . . . . . . . . . . 84

5.3 Additional Software Constraint . . . . . . . . . . . . . 86

5.4 Results . . . . . . . . . . . . . . . . . . . . . . . 87

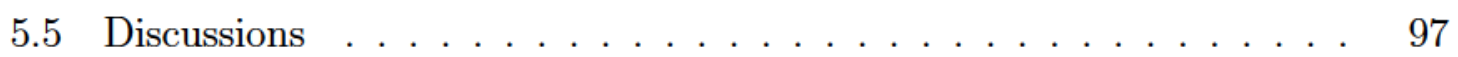

$\begin{array}{lll}6 & \text { Conclusion } & 99\end{array}$

6.1 Contributions . . . . . . . . . . . . . . . . . . . . 100

6.2 Limitations . . . . . . . . . . . . . . . . . . . . . . . . . . 101

6.3 Future Work . . . . . . . . . . . . . . . . . . . . . . . . . 102

\begin{tabular}{ll}
\hline List of References & 105
\end{tabular}

\begin{tabular}{ll}
\hline Appendices & 117
\end{tabular}

\begin{tabular}{ll}
\hline A Appendix & 118
\end{tabular}

A.1 Coherent Point Drift Registration Algorithm . . . . . . . . . . . . 118

A.2 Field of View Angle Computation . . . . . . . . . . . . . . . . . 123 


\section{List of Tables}

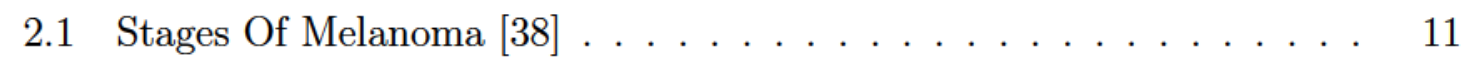




\section{List of Figures}

2.1 Normal skin anatomy showing the epidermis, dermis and subcutis lay-

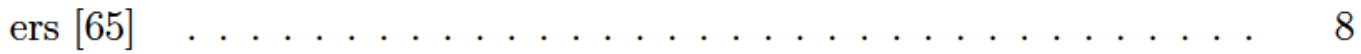

2.2 Images of benign skin lesions (left) and malignant malenoma skin lesions (right) through a dermoscope [57] . . . . . . . . . . . . . 10

2.3 Life-cycle of a 'suspicious' lesion. We added another step to the process suggested by [9] "monitoring through baseline image" . . . . . . . . 12

2.4 Pin-hole camera model. Object is placed in the origin of 3-D world coordinates $W=\left(X_{W}, Y_{W}, Z_{W}\right)$. The pin-hole coordinate system denoted by $C=(u, v, w)$. Here the $w$-axis is the camera's optical axis, and the image plane is formed focal length distance from the camera. $\quad 27$

2.5 Epipolar geometry. A point $x$ in first image could originate somewhere in the world along the ray that passes from camera's optical centre through that point, thus, it creates a constraint line (or epipolar line) $l^{\prime}$ on second image where this point could be projected. The epipoles $e$ and $e^{\prime}$ are points of intersection of a line joining camera centres $C$ and $C^{\prime}$ with corresponding camera plane. . . . . . . . . . . . . . . . . . . 30

3.1 System illustration: a. An illustration of the system assembly mechanism; camera (shown in blue), a laser range finder (shown in red) and a background board (shown in black) attached onto vertical rail-tracks;

b. An illustration of patient during scanning procedure. The patient is holding handrails, one leg is placed on the footrest, while the other is being scanned. . . . . . . . . . . . . . . . . . . . . . . . 38

3.2 Diagram of how to locate the centre of lateral malleolus bone . . . . . 45 
3.3 Platform top view . . . . . . . . . . . . . . . . . . . . 45

3.4 Platform top view after a move of angle $\theta \ldots \ldots \ldots \ldots$

$4.1 \quad$ The process for mole registration and change detection through temporal scans. . . . . . . . . . . . . . . . . 53

4.2 Intra-exploration mole mapping process flow $\ldots \ldots \ldots \ldots$

4.3 Platform top view - visible part of the leg . . . . . . . . . . . . 58

$4.4 \quad$ Voronoi cells (colourful lines) around LRF points (blue ' $x$ '), and location of moles (red 'o'). Voronoi diagram allows to locate the closest LRF point to each mole in a computationally effective manner. . . . . 62

$4.5 \quad 3 D$ leg model and image formation. Visible LRF points on the conical frustum are projected into image, as well as PSLs based on pin-hole camera model and form a $2 D$ image. The ' $x$ '-s represent LRF readings and the 'o'-s represent centre locations of the PSLs . . . . . . . . . 63

$4.6 \quad$ Platform top view - region of relevant PSLs is defined by angle $\gamma$ from optical centre. Two cases of various legs radiuses are shown . . . . . . 64

4.7 (a) Schematic representation of mole location (red dot) with relation to surrounding four LRF points (blue dots), and how we measure relative location of the mole on a $2 D$ image. (b) Projection of a 2D mole location with respect to surrounding LRF points onto a $3 D$ leg model. 68

4.8 Comparison between original simulated leg model, and calculated mole map. Lesions are identified by their ID number. ' $x$ '-s represent LRF points and 'o' represent moles. The average distance error and standard deviation in this simulation is $2.089 \pm 0.275 \mathrm{~mm} . \quad \ldots \ldots \ldots$. . . 69

4.9 Overview on temporal scan operations process $\ldots \ldots \ldots \ldots \ldots$

5.1 (a) Schematic representation of human leg [11]. (b) How to construct a shape similar to human leg with conical frustums with different opening angles attached at their bases. $\ldots \ldots \ldots \ldots$. . . . . 80 
5.2 Average accuracy with radius difference $\Delta R=10 \%$. The results are divided into groups by initial number of moles on the leg model. The scanner step size denoted by pairs of LRF and Camera step sizes $(\Delta L$, $\Delta C$ ) is varying. . . . . . . . . . . . . . . . . . . . . . . 89

5.3 Average accuracy with radius difference $\Delta R=30 \%$. The results are divided into groups by initial number of moles on the leg model. The scanner step size denoted by pairs of LRF and Camera step sizes $(\Delta L$, $\Delta C)$ is varying. . . . . . . . . . . . . . . . . . . . . . . 90

5.4 Average accuracy with scanning step size $(\Delta L, \Delta C)=\left(3^{\circ}, 10^{\circ}\right)$. The results are divided into groups by initial number of moles on the leg model. The percent of leg's radius growth in temporal scan is denoted by $\Delta R$. Overall average accuracy $95.95 \%$, minimal average accuracy per scenario type $93.40 \%$ and maximal average accuracy per scenario

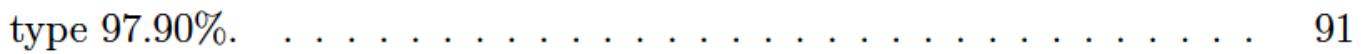

$5.5 \quad$ Average accuracy with scanning step size $(\Delta L, \Delta C)=\left(5^{\circ}, 10^{\circ}\right)$. The results are divided into groups by initial number of moles on the leg model. The percent of leg's radius growth in temporal scan is denoted by $\Delta R$. Overall average accuracy $95.36 \%$, minimal average accuracy per scenario type $92.47 \%$ and maximal average accuracy per scenario type $98.30 \%$. . . . . . . . . . . . . . . . . . . . . . . . . 92

$5.6 \quad$ Average accuracy with scanning step size $(\Delta L, \Delta C)=\left(7^{\circ}, 10^{\circ}\right)$. The results are divided into groups by initial number of moles on the leg model. The percent of leg's radius growth in temporal scan is denoted by $\Delta R$. Overall average accuracy $95.68 \%$, minimal average accuracy per scenario type $93.10 \%$ and maximal average accuracy per scenario type $97.80 \%$. . . . . . . . . . . . . . . . . . . . . . . . . 93 
5.7 Average accuracy with scanning step size $(\Delta L, \Delta C)=\left(10^{\circ}, 10^{\circ}\right)$. The results are divided into groups by initial number of moles on the leg model. The percent of leg's radius growth in temporal scan is denoted by $\Delta R$. Overall average accuracy $94.87 \%$, minimal average accuracy per scenario type $92.05 \%$ and maximal average accuracy per scenario type $96.65 \%$.

$5.8 \quad$ Average accuracy with scanning step size $(\Delta L, \Delta C)=\left(12^{\circ}, 12^{\circ}\right)$. The results are divided into groups by initial number of moles on the leg model. The percent of leg's radius growth in temporal scan is denoted by $\Delta R$. Overall average accuracy $94.43 \%$, minimal average accuracy per scenario type $91.00 \%$ and maximal average accuracy per scenario type $96.40 \%$.

$5.9 \quad$ Average accuracy with scanning step size $(\Delta L, \Delta C)=\left(14^{\circ}, 14^{\circ}\right)$. The results are divided into groups by initial number of moles on the leg model. The percent of leg's radius growth in temporal scan is denoted by $\Delta R$. Overall average accuracy $94.14 \%$, minimal average accuracy per scenario type $91.45 \%$ and maximal average accuracy per scenario type $96.40 \%$. . . . . . . . . . . . . . . . . . . . 96

A.1 Sketch of the system. . . . . . . . . . . . . . . . . . . . . . 123 


\section{Chapter 1}

\section{Introduction}

Around the world researchers collaborate to fighting cancer. Currently, extensive research is focused on developing methods to diagnose cancer at increasingly early stages. Detection of pre-cancerous signs can greatly affect the outcome course of the disease, and help eliminate the cancer altogether. One dangerous cancer type that has strong potential to be prevented during its initial stages is Cutaneous Malignant Melanoma (MM). If detected early, existing treatments can often cure the MM, with a success rate of up to $88 \%$, depending on the stage and location [38]. However, when allowed to progress into metastatic melanoma, the disease becomes far more difficult to treat. With no proven effective treatments to date, the success rate is drastically reduced to $16 \%$ or less [38, 39].

According to Canadian Cancer Statistics 2017, the number of new melanoma incidences is increasing year to year [52]. At present time, melanoma is the seventh most common cancer, and, among adolescents and adults (15-49 years), melanoma 
represents $6-7 \%$ of all new cancer incidences [52]. It is estimated that in 2017 alone, about 7,200 new cases of MM have been diagnosed in Canada, of which about 3,300 were in women [52].

Melanoma cancer can start from any cell in the human body, but some cells, such as melanocytes, are more prone for malignant transformation. The melanocyte is one of the epidermis (skin) cell types that produce the protective skin-darkening pigment melanin. Melanin has two main roles: it contributes to the skin colour, and it protects the tissue from ultra violet radiation (UVR). In the event of exposure to UVR, melanocytes raise the production of melanin. When melanocytes undergo malignant transformation, they produce MM, which is an uncommon process but very aggressive cancer [38].

$\mathrm{MM}$ is prevalent among the white population, with about 10 times greater risk than in black, Asian or Hispanic populations [38]. Other risk factors include but are not limited to: history of sunburns (especially in childhood), family history, occurrence of dysplastic nevi (or atypical moles are unusual looking benign moles), total number of benign nevi on the body (if greater than 25 the risk is doubled compared to less than 10), large nevi (more than $5 \mathrm{~mm}$ ), immunodeficiency syndrome, marked freckling on the upper back, and light hair colour [38].

Melanoma cancer has site specific prevalence, which differ between men and women. In men, the majority of Melanoma cases (57\%) appear on the torso, and only $21 \%$ of the cases appear in the lower limbs (further referred to as legs) 60]. In women, the ratio is reversed, with $41 \%$ of melanoma cases appearing on legs and only $28 \%$ on the torso 60 . 
Melanoma is eighth most common cancer for both sexes combined by projected new incidence cases in Canada as reported in [53], and the projected mortality from this disease is ranked fifteenth. In Canada, $55.1 \%$ of melanoma cases are diagnosed in stage I, while $3.9 \%$ of melanoma cases are diagnosed in the late stage IV (based on data collected during 2011-2015) [53].

Early detection of suspicious nevi requires complete skin examination, which can be performed as a part of general examination by a physician, dermatologist, community-based screening program, or even by the patients themselves [38]. In addition, dermatologists recommend tracking temporal changes of high-risk patients' skin by taking digital images [29]. Such tracking helps detect newly appearing or changing pigmented skin lesions (PSLs) [29, 24, 22]. Manual tracking of these changes is error-prone and time consuming, especially since high risk patients tend to have more than a hundred PSLs [60]. Therefore, a computer-based scanning and diagnosing system can greatly improve the quality of screening and detection of malignant moles on early stages.

\subsection{Problem definition}

This research concentrates on tracking moles in the lower limbs for two main reasons: (1) that is where the majority of MM cases occur in women [60], and, (2) the vast

majority of existing computer-based systems with a similar purpose are primarily geared for males, since they focus the screening of patients' backs $[54,74,62,59,27$, 42, 43, 44, 46]. To-date only one existing system can successfully perform total body screening [32]. Yet, even such a Total Body Scanning System (TBSS) still seems to be 
primarily geared towards screening the upper body (front and back). This happens due to the system's lack of consideration of the hidden parts of the inner legs, as well as the distortion due to the smaller radius of the legs compared to torso.

\subsection{Contribution summary}

In this research we designed and simulated a leg scanner which consists of circular rail tracks and vertical tracks using a LRF and a digital camera. The system scans the leg and collects $3 D$ measurements from the LRF and images of corresponding areas on the leg. In addition, we developed an algorithm that combines LRF measurements with skin images to create a $3 D$ leg model with moles. Last, we developed an algorithm to register moles from two temporal scans, and to track changes in mole configurations over time.

\subsection{Thesis organization}

Chapter 2 presents research that has been done on this topic in the past and is relevant to the research presented in this thesis document. In Chapter 3 we present our new leg scanner hardware that utilizes one digital camera and one laser range finder (LRF) for detection and registration of moles. In Chapter 4 we provide algorithmic solution to process resulting images and LRF readings for accurate mole registration over consecutive scans. In Chapter 5 we explain how we created simulated data and analyze lesion registration results. In Chapter 6 we give a summary of the research work presented here, and point out unresolved problems and suggestions for future 
research. 


\section{Chapter 2}

\section{Background}

The largest organ in human body is the skin, the outer covering of the body. Its main purpose is to protect us from the environment, including infections, water loss, and UVR. But the protector itself can endanger our body if not taken care of; skin can develop a deadly cancer. The deadliest among the skin cancers is MM. Fortunately, there are signs which can help detect this disease. One of the most important signs of MM are moles, or pigmented skin lesions (PSLs), medically referred to as melanocytic nevi. The presence of nevi, their shape and colour, and especially their transformations over time are all signs of potential malignancy.

In this chapter we describe the human skin structure, the formation of melanoma cancer cells, and diagnostic approaches taken by dermatologists. Finally, a survey of existing computer-aided skin scanners for MM detection is presented. 


\subsection{The Human Skin Structure}

Human skin contains several layers, each with its own distinct function [40]. The most important layers are the cellular epidermis and the underlying dermis of connective tissue (Figure 2.1). Directly underneath the dermis is a layer of subcutaneous fat. Dermis consists of two main protein fibers: collagen tissue, and elastic tissue. Collagen fibers account for the skin's tensile strength, as they are extremely tough. Elastic fibers, on the other hand, provide skin its elasticity and resilience, since they are made of elastin and elastin-associated microfibrils. The dermis layer contains blood and lymph vessels, sweat glands, hair follicles, and nerve ends. It provides nutrition to the epidermis layer, since no vessels pass through the dermal-epidermal junction [40].

The epidermis is a thin layer, typically $0.05-0.1 \mathrm{~mm}$, which consists of four sublayers [40]. The sub-layers in descending order are: (i) stratum corneum; (ii) stratum granulosum; (iii) stratum spinosum; (iv) stratum basal. The epidermis has four cell types: keratinocyte (about 95\% of total cells), Langerhans' cells, Merkel cells, and melanocytes. The keratinocyte cells are formed in the basal layer, and they move toward the upper layers while differentiating progressively. It takes about 30 days for the cells to travel from the lower level (stratum basal) to the upper level (stratum corneum). When the cells are differentiating and moving upwards, they change their

cell content. As a result, the stratum corneum layer consists of flattened cells, called corneocytes, which lost their nuclei and cytoplasmic organelles. The corneocytes are regularly removed through a desquamation (or skin peeling) process. This summarizes the cycle of skin renewal [40]. 


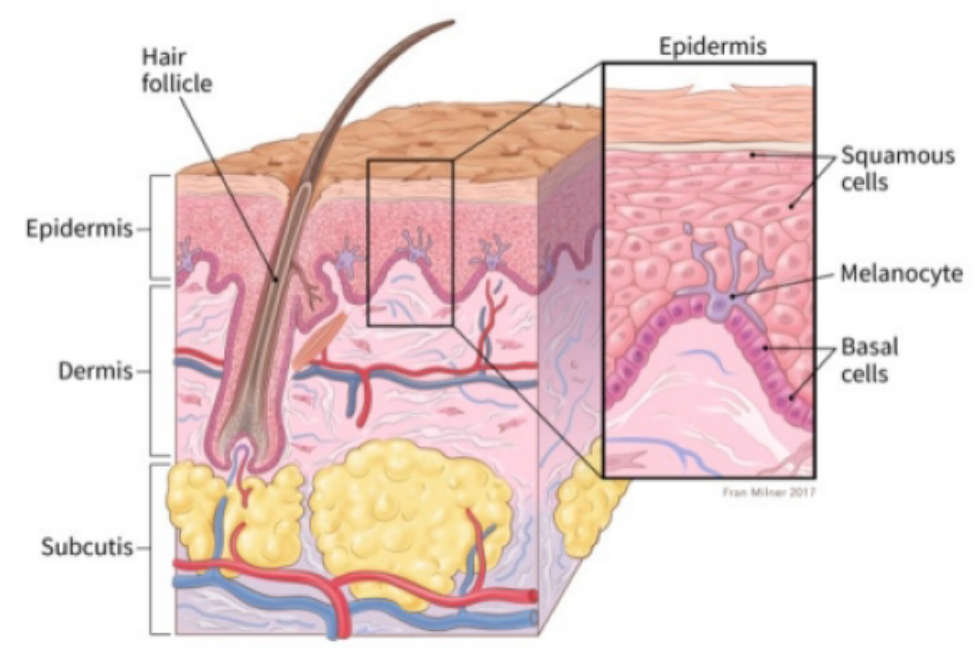

Figure 2.1: Normal skin anatomy showing the epidermis, dermis and subcutis layers 65 .

The Langerhans' cells are dentritic cells, or antigen-presenting cells. They originate from bone marrow, and they participate in the skin's immune response by detecting foreign bodies and delivering them to the local lymph nodes. Merkel cells are probably originated from keratinocytes, and they provide response to touch via mechanosensory receptors. In this thesis we are interested in the melanocytes skin cells type. These dendritic cells contain packages of melanin pigment in melanosomes which they contribute to surrounding keratinocytes. Melanin protects the subcutaneous tissue from the UVR, and contributes to the colour of skin, hair and eyes. The least common, but the deadliest, of skin cancers develops from a melanocyte cell that undergoes a malignant transformation [40]. In the following section we discuss the epidemiology and treatment of this cancer. 


\subsection{Malignant Melanoma}

Malignant melanoma is an aggressive cancer that develops in melanocytes. The number of new cases of melanoma is steadily increasing each year worldwide, even in countries with a historically low prevalence rate. Successful treatment of the disease correlates directly with its stage at the time of diagnosis. Table 2.1 demonstrates different stages of melanoma and the survival rate of treating melanoma at a certain stage. When the tumor is thin and primarily cutaneous there is a high cure rate, but for metastatic melanoma, no proven treatment has been found yet. The treatment of melanoma requires the collaboration and unique expertise of medical, surgical, and pathology professionals [38]. Figure 2.2 shows an example of benign nevi and malignant melanoma nevi as seen through a dermoscope.

A Caucasian person is at a 10 times higher risk of developing cutaneous melanoma than black, Asian, or Hispanic person [38]. Melanoma is affecting young and middleaged people, with a median age of diagnosis being fifty seven years. The frequency of occurrence of the disease increases linearly after the age of fifteen years until a peak around the age of fifty, past which point it slowly decreases [38]. Melanoma has a higher prevalence among male subjects, about 1.3 times more than in females [52]. Additionally, tumor location is sex dependent, where in male subjects, MM tumors most commonly occur on the back, however, in women they are more common on legs [60].

The major risk factors that were hypothesized to be responsible for the worldwide increase in the incidence of melanoma include increased UVR exposure due to depletion of the ozone layer and/or behavioral changes (such as usage of tanning beds and 

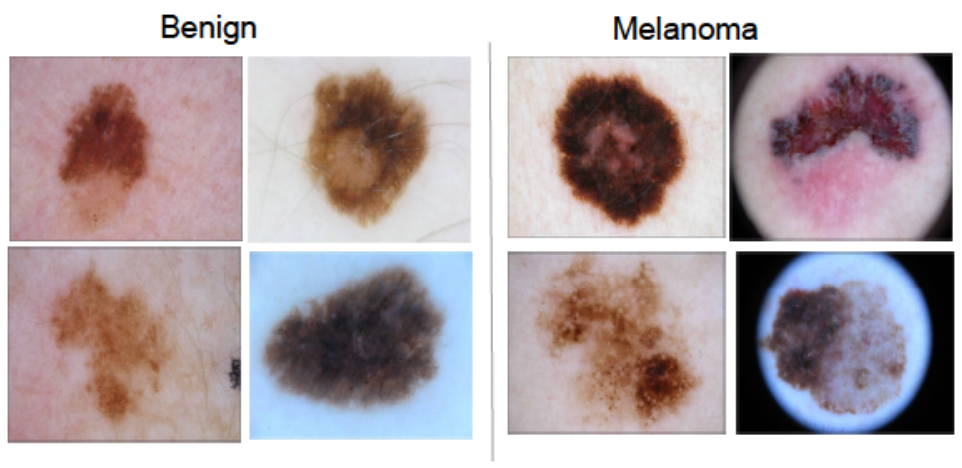

Figure 2.2: Images of benign skin lesions (left) and malignant malenoma skin lesions (right) through a dermoscope [57]

increased exposure to the sun). Other strong evidence risk factors are number of nevi, dysplastic nevi, family history of melanoma, immunosuppression, sun sensitivity or inability to tan, blue or green eyes, blond or red hair. Melanocytic nevi are benign growths of melanocyte cells and may be congenital (a mole that appears at birth) or acquired. Melanoma appears in coincidence with pre-existing nevus approximately in $25 \%$ of cases. The risk of melanoma depends on the type, size, and location of nevi. Larger nevi (more than $5 \mathrm{~mm}$ ) are associated with higher risk of melanoma. Additionally, the total number of nevi (both dysplastic and non dysplastic) is directly correlated with the risk of melanoma [38].

Dysplastic nevi (cytological atypia in melanocytic nevi) are also associated with an increased risk of melanoma. The existence of a dysplastic nevus in a person with a family history of melanoma is a significant risk marker. Patients with dysplastic nevus syndrome (or atypical nevus syndrome) have almost 100 times greater risk of developing melanoma, with about half of these patients developing melanoma by the age of fifty [38]. 


\begin{tabular}{|c|l|c|}
\hline Stage & Description & 10 year survival rate \\
\hline \hline 0 & $\begin{array}{l}\text { Melanoma in situ - cancer is fully enclosed in epider- } \\
\text { mis }\end{array}$ & Virtually 100\% \\
\hline I & $\begin{array}{l}\text { Cancer is less than 1.00 mm thick, without ulceration, } \\
\text { no metastasis to close lymph nodes or other organs }\end{array}$ & $88 \%$ \\
\hline II & $\begin{array}{l}\text { Cancer thickness is 1.01 - 4.00 mm, or greater than } \\
4 \text { mm, with or without ulceration, no metastasis to } \\
\text { close lymph nodes or other organs }\end{array}$ & $32-83 \%$ \\
\hline III & $\begin{array}{l}\text { Cancer is greater than 1.01 mm think, with or with- } \\
\text { out ulceration, with metastasis to one or more close } \\
\text { lymph nodes, it has not spread to distant sites or } \\
\text { organs }\end{array}$ & $15-48 \%$ \\
\hline IV & $\begin{array}{l}\text { Cancer has any thickness, might or might not be ul- } \\
\text { cerated, metastasis to close lymph nodes, metastasis } \\
\text { to other organs like lungs, distant skin, or far away } \\
\text { lymph nodes }\end{array}$ & $3-16 \%$ \\
\hline
\end{tabular}

Table 2.1: Stages Of Melanoma 38]

\subsection{Diagnosis of Melanoma}

The ultimate way to diagnose melanoma is tissue excision (biopsy) and pathological diagnosis of specimen [38]. However, it is in a clinician's hands to determine which lesions could be malignant, and thus require extraction. Suspicious lesion life-cycle is outlined in Figure 2.3. Various methods have been developed, such as the ABCDE rule [2, 68] and 7-point checklist [35], that guide clinicians for lesion examination. There are various optical devices that can help the dermatologist have a better look at the lesions, including the dermatoscope. This chapter outlines those methods and devices. 


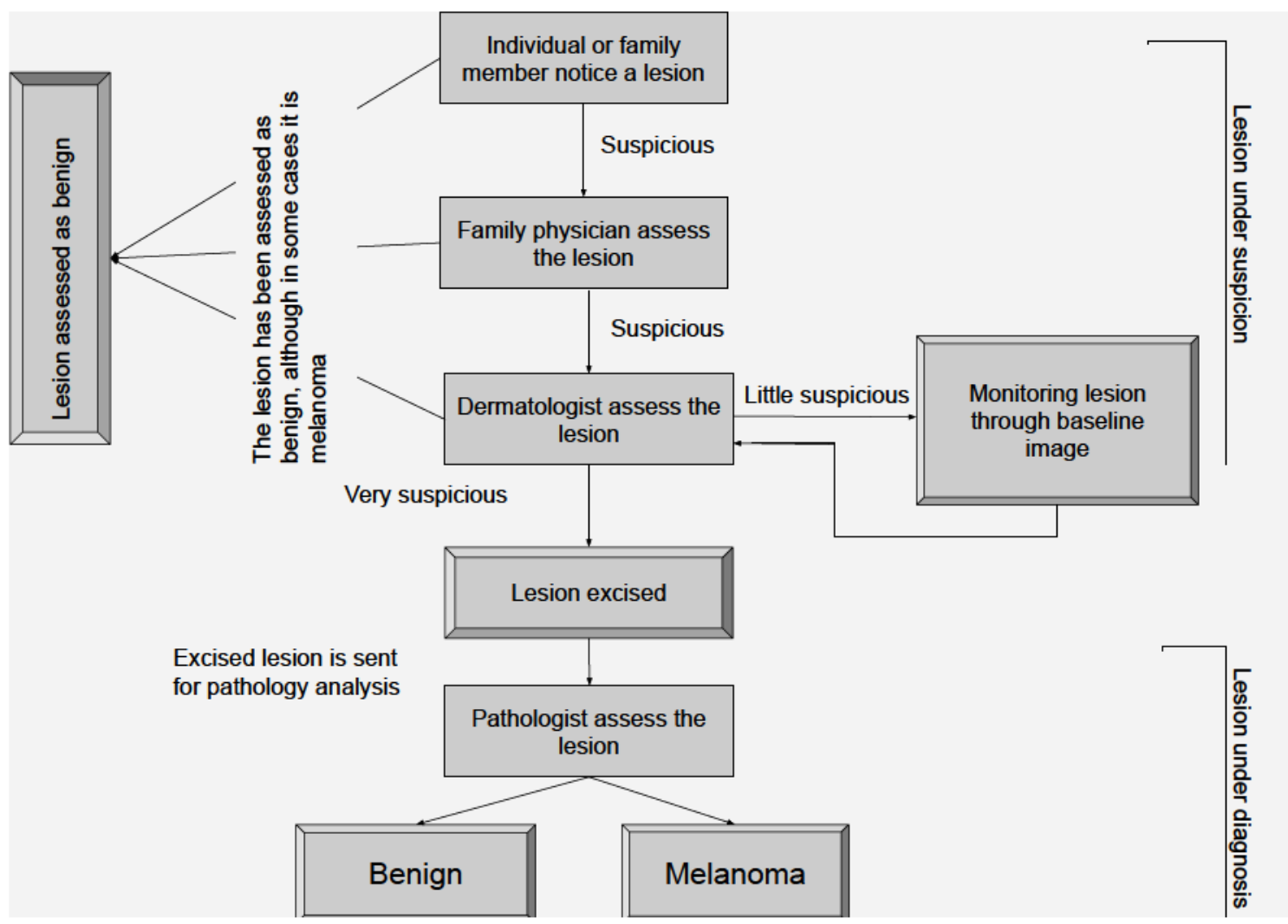

Figure 2.3: Life-cycle of a 'suspicious' lesion. We added another step to the process suggested by [9] "monitoring through baseline image" 


\subsubsection{Naked eye - ABCDE rule}

Malignant pigmented lesions have features that differ from healthy ones. Thus, an ABCD guide was designed by Friedman et al., [20] three decades ago to help medical professionals, and even patients themselves, identify the suspicious lesions. This acronym stands for Asymmetry, Border irregularity, Color Variation, Diameter (greater than $6 \mathrm{~mm}$ ). Furthermore, early melanoma lesions were noted for the rapid changes they experience, thus a letter E (for evolution or enlargement) was added in 2004 and refers to significant change in size, shape, shades of colour, surface (raised, bleeding, crusting), or symptoms (itching, tenderness) [68]. The purpose of the mnemonic is to create a simple memorable tool. As a result, although it is a highly useful tool, it does not represent the full list of melanoma characteristics. Pigmented lesions do not need to have all the ABCDE attributes to raise suspicion for malignancy, and the suspicion level rises with each additional ABCDE attribute which characterizes the lesion [68].

Based on a prospective study [67], where each criteria of the ABCDE rule was tested individually the sensitivity ${ }^{1}$ was $57 \%, 57 \%, 65 \%, 90 \%$ and $84 \%$, respectively. The specificity $\left.\right|^{2}$ of each criteria for melanoma diagnosis compared with other pigmented tumors was $72 \%, 71 \%, 59 \%, 63 \%$ and $90 \%$ for ABCDE, respectively. Note the importance of criteria $\mathrm{E}$, which gives high sensitivity and specificity results. When two criteria are present simultaneously the sensitivity and specificity of diagnosis of melanoma are $89.3 \%$ and $65.3 \%$, respectively. When three criteria are present the

\footnotetext{
${ }^{1}$ Sensitivity, or true positive rate, is defined by number of sick people correctly identified as sick divided by number of sick people in tested group.

${ }^{2}$ Specificity, or true negative rate, is defined by number of healthy people correctly identified as healthy divided by number of healthy people in tested group.
} 
sensitivity and specificity of diagnosis of melanoma are $65.5 \%$ and $81 \%$, respectively (when three criteria are present, the false positive rate goes down, however, the false negative rate goes up).

\subsubsection{7-point checklist}

The 7-point checklist method was also designed to simplify the diagnosis of malignant pigmented lesions [3, 15]. The check list is defined by three major criteria:

1. atypical pigmented network

2. blue-white veil

3. atypical vascular pattern

And four minor criteria:

1. irregular streaks

2. irregular pigmentation

3. irregular dots/globules

4. regression

Major criteria have a score of two, while minor criteria have a score of one. When a lesion scores more than three points (for example, one major and one minor criteria, or three minor criteria) it is diagnosed as melanoma. A recent study analysis reported $87.5 \%$ sensitivity, $65.9 \%$ specificity and $70.4 \%$ diagnostic accuracy of the 7 -point checklist method [69]. 


\subsubsection{Optical Diagnosis}

\subsubsection{Dermoscopy}

In the 1990s, a hand-held device was introduced which allowed a surface microscopy of a PSLs with a better diagnostic accuracy [61]. The technology is called Dermoscopy (also known as Epiluminescence Microscopy or ELM, Dermatoscopy). The dermotoscope is equipped with a magnifying glass, light beam and an application liquid (i.e., gel, oil, or alcohol). The liquid reduces light reflection and makes the epidermis translucent, allowing in vivo visualization of subsurface anatomical structures. Newer instruments eliminate the need of liquid by use of cross-polarizing light filters. Direct contact dermoscopy offers increased illumination and increased resolution as compared with polarized dermoscopy. In addition, the colours seen under direct contact are sharper and less distorted than with polarized light [37]. Literature review reveals that, although dermoscopy characterizes nevi with increased sensitivity and specificity, it is highly dependent on the experience of the medical professional [61]. Additionally, clinicians may be discouraged from using dermoscopy because they feel it is too time consuming [61].

\subsubsection{Transillumination technique - Nevoscope}

Transillumination is a new method of ELM imaging [76]. The light is directed from a circle around the lesion towards its center at an angle of $45^{\circ}$. The light accumulates at a focal point about $1 \mathrm{~cm}$ below the skin, forming a virtual light that makes the subsurface of the skin translucent. This technique allows a view of PSL subsurface with a good sensitivity to imaging vascularization and an increased blood flow. The 
device was patented and called Nevoscope [10], but has not been widely used in clinical practice.

\subsubsection{Baseline Imaging}

Baseline imaging is a concept of comparing a cutaneous lesion to its image taken in the previous examination [4, 30]. The concept refers to any means of image acquisition including dermoscopic. As noted in the study [4], nevi are dynamic, but only a small portion of the newly appearing or changed lesions are melanomas. Acquiring baseline images allows easy change detection. A study [30] showed that baseline images help detect even featureless melanoma, thus, increasing the chances of catching melanoma while it is yet non-invasive.

\subsubsection{Total Cutaneous Photography}

Most dermatologists believe that baseline photography (digital or not) is useful in the follow-up management of pigmented lesions [37, 50]. Obtaining baseline single or multiple skin lesion photographs can prove helpful in the early detection of melanoma. Clinical images of PSLs allow mapping their location on the human body and tracking their changes over time, thereby alerting physicians of early melanoma.

Total body photography, also known as total body skin imaging (TBSI) and wholebody photography (WBP) is a method of photographic documentation of a person's entire cutaneous surface through a series of body images [37]. During TBSI, a patient should follow a set of standardized body poses [25]. This method requires additional considerations such as camera, lights, image storing system. A computer software 
solution, which attempts to provide automatic comparison between two consecutive total body photography screening sessions, and highlight newly appearing or changing PSLs, is of potential benefit in clinically following up with patients who have multiple melanocytic nevi.

Advantages of WBP include [25]:

- Detection of newly appearing or changing skin lesions without depending on classical clinical features [16].

- Avoidance of biopsies in suspicions but stable lesions, as confirmed in study conducted by Feit et al., [16].

- Efficient assessment of patients with a substantial number of lesions.

- Independence from expert interpretation of dermoscopic images.

- Development of photography and software aids for acquisition, archiving and comparison of consisting whole-body skin images is cost-effective

- Long-term cost-effectiveness, achieved through the development of photography and software aids for acquisition, archiving, and comparison of consecutive whole-body skin images.

For better accuracy and efficiency, WBP should be combined with other techniques, including dermoscopy [25]. A study conducted by Rademaker et al., [58] concluded that WBP followed by digital dermoscopy results in detection of thinner melanomas, as compared with traditional diagnostic methods. 
A study surveying the use of WBP [66] found a modest increase in integration of the technology in dermatology residency programs compared to that of dermoscopy. The study concluded that treatment of patients with many atypical nevi (more than five) is the most common reason for the use of WBP [66]. The study also noted that the use of WBP helps to find melanoma in an early curable stage, leads to fewer biopsies, reduces patient's anxiety, and reduces cost of care through early diagnosis. In addition, the study found that digital photography imaging improves record keeping. However, lack of training, as well as logistical and financial constraints were mentioned as obstacles for a wider application in clinical practice [66].

\subsubsection{Computer Aided Diagnosis}

Despite the use of above mentioned diagnostic algorithms and assistive devices such as dermatoscope for melanoma diagnosis, it is still a challenging process and accuracy is considered to be limited [71]. The meta-analysis by Malene E Vestegaard, et al. [71] demonstrated that dermoscopy improved clinicians' decision, compared to naked-eye, with their ability to correctly identify melanoma lesions out of all PSLs, with sensitivity of $79 \%$ for dermoscopy versus $54 \%$ for naked eye, and specificity $71 \%$ for both dermoscopy and naked eye. Furthermore, clinical diagnosis of melanoma depends on dermatologist's experience, is very subjective, and suffers from inter- and intra-observer variations. Researchers started to develop systems for the automated diagnosis of melanoma or Computer-Aided Diagnosis (CAD) with intention to replace dermatologist's decision with regards to PSLs images interpretation. The ideal

system would increase the sensitivity and specificity of the diagnosis, thus saving lives and reducing the number of unnecessary surgeries, ultimately reducing medical 
and emotional costs of biopsies. Despite such systems being developed for a variety of imaging modalities, [36, 70] this research focuses only on automated melanoma recognition systems based on clinical photography and dermatoscopy.

Due to improvements in imaging technologies and image processing techniques in recent years, there has been a significant increase of interest in the development of computer-based diagnostic system for melanoma and the first research started in mid 1980s. The generic CAD system would (i) perform image pre-processing; (ii) locate the lesion(s) by border identification; (iii) extract dermatological features of the lesion(s); (iv) classify and diagnose lesions.

There is a lot of research and improvement in CAD systems for melanoma screening in experimental settings. However, the practical value of such systems is still unclear [31]. Even though a patient would accept computer-based analysis for melanoma screening, currently it cannot be recommended as a replacement for a dermatologist decision due to its tendency to over-diagnose benign as malignant (i.e., false positive) melanocytic lesions and non-melanocytic skin lesions [21]. Furthermore, in general, CAD systems have two inherited drawbacks in their development [9]:

1. CAD systems are expected to reproduce pathologists' decisions (i.e., decide if the lesion is melanocytic or not) with only the input of dermascopic/clinical images which is insufficient information for the diagnosis. In contrast, the ideal CAD system should reproduce the decision of a dermatologist (i.e., define the level of possibility for the lesion to be malignant) and provide an explanation for that decision [12]. 
2. Histopathological data is available only for lesions that were considered suspicious by dermatologists. In other words, moles that did not raise dermatologists' subjective suspicion could not be used for feature extraction by CAD systems, because their state (i.e., benign or malignant) is unknown.

A curious reader can read a review on single lesion CAD systems progress by Korotkov, et al. [31].

\subsubsection{Automated Multiple Lesion Diagnosis}

Total body skin examination (TBSE) plays an important role in identifying melanocytic or potentially malignant PSLs. An important risk factor for melanoma is a high number of PSLs (more than fifty). Thus, the task of skin examination is very tedious and time consuming for dermatologists. Going over all the lesions, checking more closely for signs of melanoma the suspicious lesions, and comparing them with baseline images for signs of evolution is a difficult task. Besides, it is prone to errors like missing a suspicious mole. Comparison with baseline photographs is not as simple as it sounds:

- Images could be taken from different angles, thus comparison with a baseline image for evolution becomes tricky.

- The mole could have been missed during baseline photography.

- The mole could have evolved since the baseline photography and now it is difficult to recognize the corresponding image.

- An entirely new mole could have appeared. 
An automated WBP could potentially eliminate all those problems and make the process of TBSE faster and cheaper, which is very important for early melanoma detection based on the evolution of moles. Automated multiple lesion analysis is a challenging process due to the trade-off between resolution and body coverage per image, where both factors can influence change detection.

Although, it is clear that automated TBSI is a prospective solution for early melanoma detection, the subject has not yet received appropriate attention among researchers. In this section we summarize the existing literature on the analysis of multiple PSLs. Existing research on automated WBP can be divided into four categories:

1. Image storage system - WBP and image storing without any computer analyses, allowing medical professionals easy access to baseline images

2. Lesion localization - multiple lesion photography with only lesion localization, i.e., identifying the lesions on the image

3. Lesion registration - automating the process of "registering" or matching lesions on a pair of images, for the purpose of lesion comparison and change detection over consecutive scans

4. Lesion localization and registration - most advanced systems that automatically estimate dimensional change of lesions 


\subsubsection{Image Storage Systems}

One example of commercially available technology for WBP is Melanoscan ${ }^{\circledR}[14,13]$. Melanoscan ${ }^{\circledR}$ is a photo-therapy room with a 25 digital camera array. A patient is scanned in several body poses, which can be easily reproduced. The images are stored encrypted. A software allows easy access, enlargement of area of interest, and comparison of images in consecutive scans by health professionals. The scanning process is automatic, but there is no automatic image processing for lesion localization or registration purposes.

\subsubsection{Mole Localization}

In 1989, Perednia et al, [55] showed how a bilaterally symmetric Laplacian-of-Gaussian filter can be used to detect borders of multiple lesions on digital images. They assume that on images with low image resolution per lesion, the noise like hair and shadows is alleviated. In 1991, White et al. used the concept of "3-D brightness pits" in order to find features on skin images [75]. The identified pits are used for feature extractions, such as area, perimeter, total volume and more. Then Fisher's multivariate descriminal analysis [51] and K-th nearest neighbor [63] classification methods were used to distinguish skin lesions and identify them on the images. In 1995, The authors Filiberti, et al. [17, 18] transform a digital image into hierarchical segmentation. This pyramid representation of the image is given to a neural network classifier to find lesions.

In 2005, Lee et al, developed an algorithm to count moles automatically on digital images of the back 34]. There are three main steps in the algorithm: pre-processing, 
using mean shift filter, and choosing possible moles candidates. True moles are selected based on features like size, contrast, and elliptical shape. The resulting sensitivity of the algorithm is $91 \%$ [34].

In 2007, Cho et al, presented a system that detects moles on skin images, not only back torso [8]. The system's main stages are: skin detection, hair removal, possible moles detection by difference-of-Gaussian filters. The localized spots on the skin are identified as moles or not moles by a trained support-vector machine.

\subsubsection{Mole Registration}

In 1992, Perednia and White et al, [74, 54] developed computerized matching of lesions that can help register changes of moles. Initial matching points are found using the "Gabriel graph" algorithm on pairs of images, and the result is used in a point pattern matching algorithm in order to pair all other lesions. With 3 initially matched points, the performance of the point pattern algorithm on a subject under realistic conditions is about $97 \%$ accurate.

In 1998, Mcgregor and Bruce invented a new algorithm for automatically registering PSLs on paired images based on four steps: first it generates a lesion map on both digital images, then it finds basic matching lesions, next it selects corner lesions,

and the last step is to rotate and resize the images in order to align them [41]. It successfully registered PSLs on paired images that differ in total number of lesions, and observed geometry. In a different research from 1998, Roning et al. [62] introduce an algorithm for lesion registration in successive skin images based on the geometrical properties of the lesions. The algorithm requires an input of two matched moles to 
begin with. At each exploration step it chooses the next closest point to the baseline for matching. It also accounts for the exceptional points that don't have matching pairs.

In 2007, Huang et al. proposed a melanoma registration algorithm which is based on bipartite graph matching problem [27]. In order to measure similarities between lesions and to build the bipartite graph, Voronoi cells are used. The overall correspondences between dermatological images are found through minimum weight maximum cardinality matching. The result algorithm was tested on dermoscopic images of the patient's back.

Mirzaalin et al. in 2009, proposed a method of mole registration in images of the patient's back taken at different times [42]. A human back template is introduced for creating a spatial coordinate system. Moles are matched on the coordinate system as a graph matching problem, where nodes and edges play an important role in the matching cost function. In 2012, Mirzaalin et al. developed Skin Lesion Matching Using a High Order MRF Optimization Framework [43]. The authors formulate the PSL registration/matching problem as relaxed labeling of an association graph based on high order Markov Random Field energy. In order to find a solution with low uncertainty they introduced an entropy energy term into the objective function. In 2014, this group of researchers proposed to create a coordinate system for the human back based on anatomical landmarks as reference points on the back images [44]. The landmarks allow one to construct a grid system, and locate the PSLs in this grid. They compared six state-of-the-art PSL matching methods with and without grid systems, in order to provide strong evidence that the system improves the matching procedure. In 2015, they invented another algorithm for moles matching on digital 
images of human back [45]. The input of their algorithm consists of two graphsrepresented sets of PSLs. The algorithm assigns energy terms for the PSL matching objective function. It matches triplets of vertices from one graph to another by quantifying topological-preservation using the Jacobian matrix method.

\subsubsection{Mole Localization and Registration}

In 1995, Holger Voigt et al., introduce a topodermatographic algorithm to automatically estimate dimensional changes of PSLs [73]. This technique analyzed changes of skin-lesions over a series of high-resolution digital video images. The system captured only front and back torso. Resulting images were processed by algorithms (the article provides very vague description of them), such as mathematical filters and Sobel operators for lesion localization. Relational search algorithms were applied, instead of overlaying images for comparison analyzes (due to axial distortion) and a digitized comparison of sequential images was performed to detect new, missing (excised lesions), and existing lesions, and highlighting significant changes. No further work on technique improvement or implementation in clinical practice has been reported.

In 2016, Mirzaalin et al, introduced an end-to-end PSL tracking system on matching back images [46]. The input is a pair of skin back images and the output is matching lesions and identifying the newly appearing and disappearing ones. First, it detects anatomical landmarks using a pictorial structure algorithm. Second, lesions are detected, and associated with the spatial properties of landmarks. Last, by labeling an association graph using tensor-based algorithm the lesions are matched. All these steps are performed with a structured support vector machine and an adaptive learning approach. 
In 2015, Korotkov et al. , invented a total body scanning system based on the photogrammetry concept [32]. The scanner consists of a chamber with 21 highresolution cameras, cross-polarized light and a turntable. The scanner automatically acquires a set of overlapping images in two body positions, covering $85 \%-90 \%$ of the patient's skin surface. An algorithm allows mapping of the PSLs and change detection between explorations. Images of individual lesions are mapped with body-to-image and image-to-image correspondence. Initial results obtained from the scanner showed that an automated temporal monitoring of multiple lesions was successful.

\subsubsection{Summary}

We presented the previous research in the field of computer based multiple lesion diagnosis over successive scans. We noticed that most of the presented research was primarily geared towards men's health, since it concentrated on tracking moles on back skin images. Automating nevi tracking on legs, which is utmost important for women's health (since for women melanoma is prevalent on legs) was present only in one research by Korotkov et al. [32]. Even though this scanner covers about $85 \%$ of human skin, we suspect that, resulting images of inner thighs are out of focus, or totally missing (since they are hidden to the cameras in the provided scanning poses). In addition, this system does not provide a comprehensive solution for body changes between successive scans. This is an important aspect for a scanner that will be used with sometimes prolonged periods between scans. During these periods legs can change in their size and shape (for example due to water retention). Therefore, we conclude that a specialized scanner for tracking nevi on legs, that is robust to constant legs' shape changes is missing. 


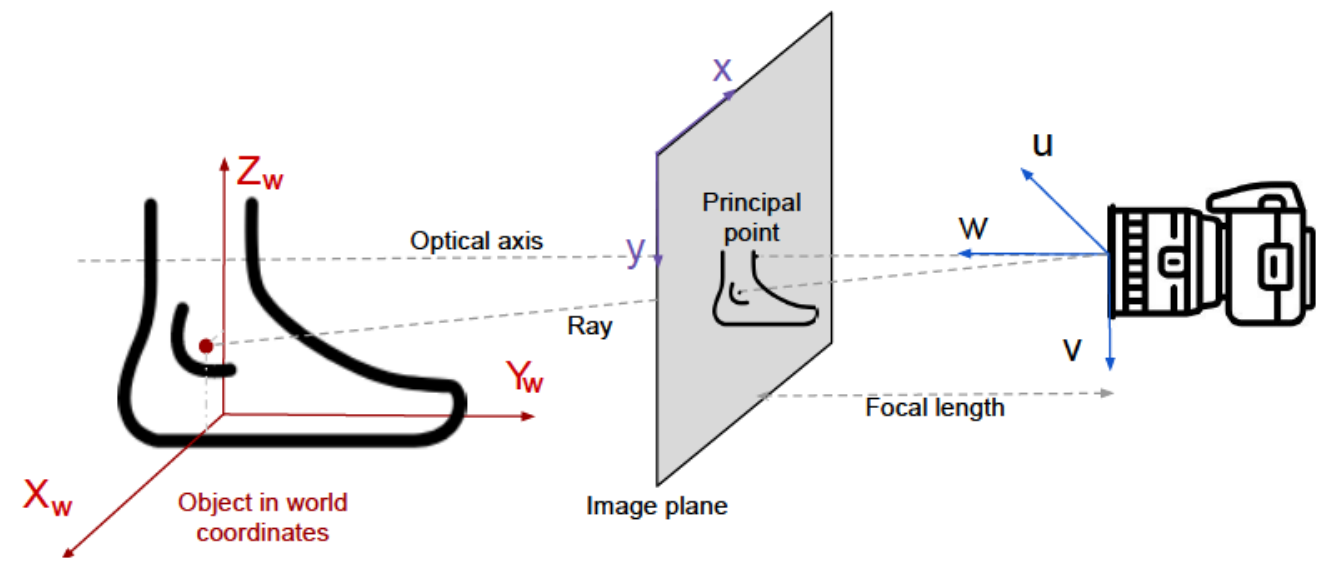

Figure 2.4: Pin-hole camera model. Object is placed in the origin of 3-D world coordinates $W=\left(X_{W}, Y_{W}, Z_{W}\right)$. The pin-hole coordinate system denoted by $C=$ $(u, v, w)$. Here the $w$-axis is the camera's optical axis, and the image plane is formed focal length distance from the camera.

\subsection{Computer Vision}

\subsubsection{Pinhole Camera Model}

A pinhole camera model is a mathematical model that describes image formation. The camera is described as a box with a small hole in the front [56]. Light is scattered from an object in the world and passes through the hole. An inverted image is formed on the back surface of the box. A mathematically equivalent model can be simulated with a virtual object in front of the camera box in the upright state, Figure 2.4 .

The world coordinates are represented by $W=\left(X_{W}, Y_{W}, Z_{W}\right)$. The camera coordinate system denoted by $C=(u, v, w)$. The Focal length is the distance between camera's optical centre and the principle point of the image, Figure 2.4. The image coordinates are denoted by $X_{c a m}=(x, y)$. The relation between a world point in 
camera coordinate system, $C$, and an image point, $X_{c a m}$, is denoted by:

$$
\begin{aligned}
& x=\phi_{x} \frac{u}{w} \\
& y=\phi_{y} \frac{v}{w},
\end{aligned}
$$

Where $\phi_{x}$ and $\phi_{y}$ are the focal scaling factors of distance to pixels in $x$ and $y$ directions respectively. The initial pixel position $X=(0,0)$ in most imaging systems is at the top left corner, but in our model we expect the initial point to be in the centre of the image. Thus, we need offset parameters, $\delta_{x}$ and $\delta_{y}$ (in pixels), to move the starting point to the centre of the image. An additional skew parameter, $\gamma$, is required for a final model, since the spacing of photo-receptors are different in $u$ and $v$ directions.

The resulting camera intrinsic matrix is $K$ :

$$
K=\left[\begin{array}{ccc}
\phi_{x} & \gamma & \delta_{x} \\
0 & \phi_{y} & \delta_{y} \\
0 & 0 & 1
\end{array}\right]
$$

For easier mathematical representation of these relations, we consider homogeneous coordinates, where the conversion between Cartesian and homogeneous coordinates is done by appending one to the original vector: $\tilde{X}=\lambda\left[\begin{array}{l}x \\ y \\ 1\end{array}\right]$. The world 
coordinates are $\tilde{C}=\lambda\left[\begin{array}{l}u \\ v \\ w \\ 1\end{array}\right]$. Where $\lambda$ is an arbitrary scaling factor.

For a full model, we also need to take into account the camera extrinsic parameters which are the translation vector, $\vec{t}$, and the camera's orientation matrix, $\boldsymbol{R}$. These translate the world coordinate system into the camera coordinate system.

$$
\lambda\left[\begin{array}{l}
x \\
y \\
1
\end{array}\right]=\left[\begin{array}{ccc}
\phi_{x} & \gamma & \delta_{x} \\
0 & \phi_{y} & \delta_{y} \\
0 & 0 & 1
\end{array}\right]\left[\begin{array}{llll}
R_{11} & R_{12} & R_{13} & t_{1} \\
R_{21} & R_{22} & R_{23} & t_{2} \\
R_{31} & R_{32} & R_{33} & t_{3}
\end{array}\right]\left[\begin{array}{l}
u \\
v \\
w \\
1
\end{array}\right]
$$

Or in a short matrix form:

$$
\lambda \tilde{X}=\boldsymbol{K}(\boldsymbol{R} \tilde{C}+\vec{t})
$$

The explanation was inspired by [56].

\subsubsection{Epipolar Geometry}

When one still scene is captured by a single camera from each of two different locations, a new geometric relationship is formed, allowing the reconstruction of the $3 D$ scene from a pair of $2 D$ images (up to a scale). Any point, $x$, from the first image is 


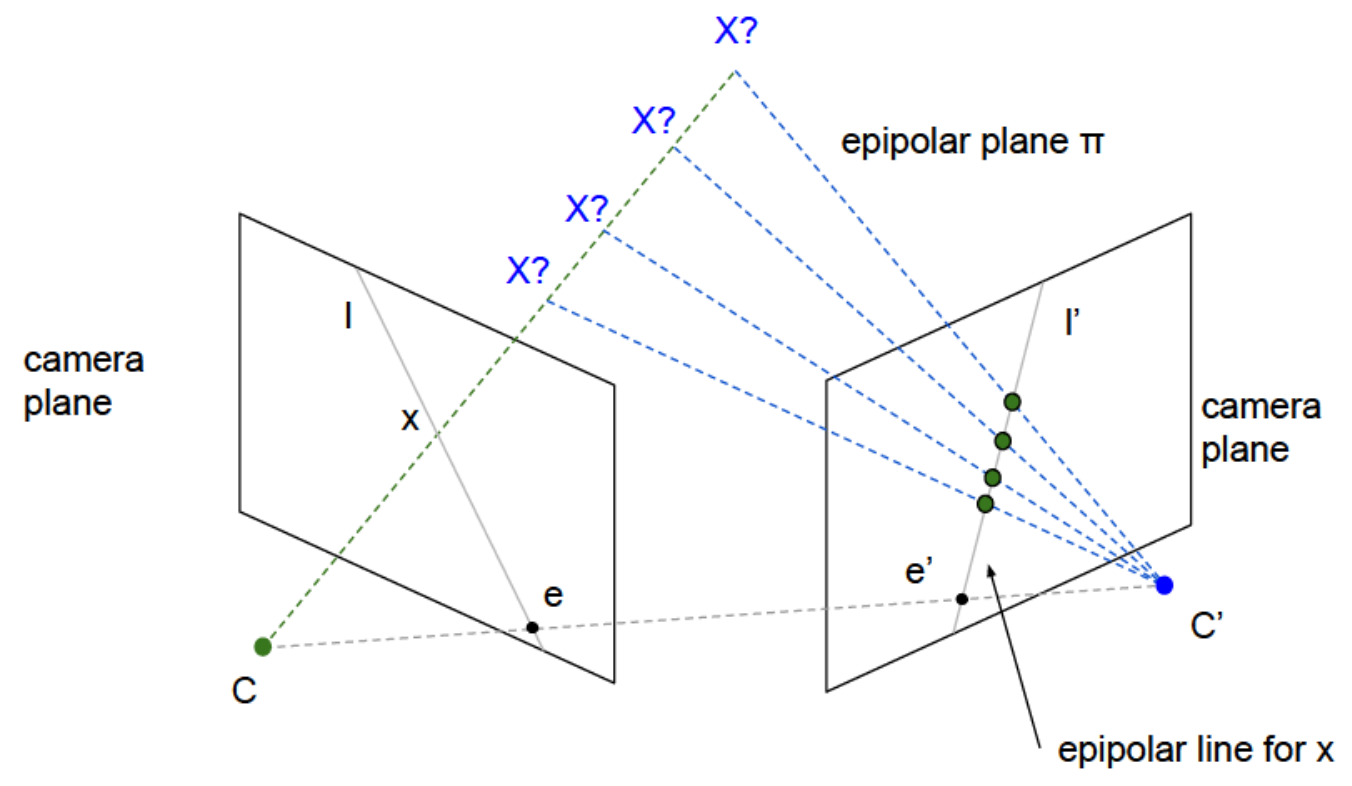

Figure 2.5: Epipolar geometry. A point $x$ in first image could originate somewhere in the world along the ray that passes from camera's optical centre through that point, thus, it creates a constraint line (or epipolar line) $l^{\prime}$ on second image where this point could be projected. The epipoles $e$ and $e^{\prime}$ are points of intersection of a line joining camera centres $C$ and $C^{\prime}$ with corresponding camera plane.

constrained to lie on a line, $l^{\prime}$, from the second image (Figure 2.5). This is known as epipolar constraint.

A point, $x$, from the first image is related to point $x^{\prime}$ from the second image by the relationship $x^{\prime}=H_{\pi} x$ ( $H_{\pi}$ is a transfer mapping from first image to second image via any plane $\pi$ ). At the same time, epipolar line, $l^{\prime}$, is formed from connecting epipole point $e^{\prime}$ with $x^{\prime}$, and this relationship can be written as

$$
l^{\prime}=e^{\prime} \times x^{\prime}=e^{\prime} \times H_{\pi} x
$$


We define a fundamental matrix as $F=e^{\prime} \times H_{\pi}$ (note that $\mathrm{F}$ is rank 2 matrix). And the relationship between epipolar line $l^{\prime}$ and a point $x$ is defined by

$$
l^{\prime}=F x
$$

The fundamental matrix also satisfies the condition:

$$
x^{\prime} F x=0
$$

It is interesting to note that fundamental matrix $F$ has eight degrees of freedom. Thus, by finding a minimum of eight points' correspondences on two images, a fundamental matrix for the images can be calculated.

We also define a $3 \times 3$ essential matrix, $E$. This matrix captures the relationship between the two view points of the camera through a translation vector, $t$, and rotation matrix, $R$. This matrix's properties are: $\operatorname{rank} 2, \operatorname{det}|E|=0$, and only 5 degrees of freedom.

$$
E=t \times R
$$

An essential matrix can be also derived from a fundamental matrix by

$$
E=K^{T} F K
$$

While $K$ is the intrinsic camera matrix. 
In our calculations we utilized P. Kovesi [33] implementations of these concept on Matlab platform [1]. The above explanation was inspired from the books: "Multiple view geometry in computer vision" [26] and "Computer vision: models, learning, and inference" [56].

\subsubsection{D Registration Algorithm}

We researched different $3 D$ registration algorithms and chose to use the Coherent Point Drift (CPD) algorithm by Myronenko et al. [47, 48] for this research. We chose this method due to (i) its accuracy in $3 D$ point sets registration and (ii) its fast implementation that reduces the system's complexity to linear (the implementation is available online [49] on Matlab platform [1]). Appendix A.1 outlines the algorithm in more details.

The CPD algorithm examines alignment of two point sets as a probability density estimation problem, where the first point set represents the Gaussian Mixture Model (GMM) centroids, and the second one represents data points. The algorithm output is a correspondence map $C_{12}$ of optimal alignment from the first point set $S_{1}$ to the second point set $S_{2}$. The outliers of $S_{1}$ (i.e., points $o_{i} \in S_{1}$ that the algorithm does not find a match for in set $\boldsymbol{S}_{\mathbf{2}}$ ) are omitted from the correspondence map. That is, if we denote the first column of correspondence map $C_{12}$ by $C_{12}^{1}$, then $o_{i} \notin C_{12}^{1}$. All points from the $b_{i} \in S_{2}$ are still present in the correspondence map $C_{12}$, i.e., $S_{2} \cap C_{12}^{2}=S_{2}$ (here $C_{12}^{2}$ denotes the second column of correspondence map $C_{12}$ ). Therefore, the resulting correspondence map is a one-to-many match. Figure 2.10 is an example of resulting correspondence map without loss of generality. 


$\begin{array}{cc}a_{1} & b_{1} \\ \vdots & \vdots \\ a_{i-1} & b_{j-1} \\ a_{i} & b_{j} \\ a_{i} & \vdots \\ a_{i} & b_{j+k} \\ a_{i+1} & b_{j+k+1} \\ \vdots & \vdots \\ a_{N} & b_{M}\end{array}$

\subsection{Summary}

In this chapter we provided background knowledge to better understand melanoma skin cancer and the tools currently available to diagnose it. We explained the skin structure, and how cellular distortion leads to melanoma. Moreover, we looked into existing medical practice of melanoma diagnosis through medical diagnosis algorithms and assistive optical technology. Then we explored two research directions in computerized diagnosis: one through diagnosis of a single lesion by feature extraction, and the other through multiple lesion digital photography, localization, and registration of PSLs for the purpose of lesion change detection. This research is all about creating new means for early melanoma detection in the lower limbs, while existing multiple PSLs diagnosis research mainly concentrates on the human torso, or just the human back. At last, we reviewed concepts of photogrammetry, useful for the analysis of multiple images of one scene from different locations, taken by one digital camera. 
In the next two chapters we propose and detail our leg scanner system for melanoma detection. 


\section{Chapter 3}

\section{Lower Limbs Scanner Hardware}

\section{Design}

The most reliable sign of nevus malignancy is the change and evolution of a mole in terms of size, shape, or colour [2]. Therefore, it is recommended to track the state of each PSL via baseline images [64]. During a follow up TBSE a physician could refer to previously registered PSLs states and identify "evolving" lesions through comparison. Manual comparison with baseline images is tedious and error prone and with progress of digital cameras, computational capabilities and computer vision algorithms, that task can and should be automated.

As mentioned in Section 2.2, women experience more melanoma growths on their legs than on any other body part. It is easy to notice that the legs - the body part that is most significant for women's health in the context of melanoma - is poorly covered in all previous automatic and semi-automatic systems (Section 2.3.5.4) as 
they concentrate on the human torso. Therefore, in this research we decided to develop an automatic leg scanner for melanoma detection.

A leg scanner for melanoma detection should take into account the following aspects:

- When capturing images of a leg, there is a trade-off between skin coverage per image and image resolution. High-resolution images are necessary to receive fine details of PSL in order to detect changes over time. However, we want to achieve higher skin coverage per image for faster scanning process.

- A leg has circular symmetry. Hence, the images should be taken from various angles around the leg; ideally, images should be taken with the camera's optical centre aligned perpendicular to the skin. Fulfilling this condition will minimize the distortion of the PSLs in the captured images.

- Taking images of inner thighs with the camera's optical centre perpendicular to the skin surface is challenging, since the other leg interferes with creating enough space for the camera.

- Since a leg has a circular symmetry, a starting point for leg capturing must be decided.

- Human legs have a tendency to change in shape over time due to muscle changes, water retention, and changes in amount of fat. As a result, the $3 D$ locations of moles with respect to the starting point (i.e., first scan) are constantly changing.

In this research we propose an innovative design and technique of a semi-automatic screening for early detection of melanoma, which will potentially improve diagnostics 
and result in a good outcome, particularly for women. The scanner produces images of PSLs from various angles and heights and a $3 D$ model of the leg (reconstructed from LRF readings). These results provide grounds for an accurate reconstruction of the subject's mole map on a $3 D$ leg model, and lesion registration across consecutive explorations for finding changing or newly appearing PSLs.

Further along in this chapter, we describe the envisioned design in Section 3.1 and the process of patient scanning in Section 3.2. We also discuss the envisioned system's limitation due to the system dimensions and structure in Section 3.3. The chapter is finalized with conclusions (Section 3.4).

\subsection{Hardware design}

The envisioned scanning system is assembled around a circular platform, as shown in Figure 3.1. The platform surface is marked for the heel placement, such that the heel's centre is approximately aligned with the centre of the platform. A circular rail track surrounds the platform and is concentric to it. The design also incorporates handholds, and a footrest for the leg that is not being scanned.

For scanning purposes, the patient must stand on one leg, while the other leg is lifted to an almost horizontal position and placed on the footrest Figure 3.1. Handholds are positioned at each side of the patient for balance, such that the patient is able to stand on one leg more easily and almost motionlessly for the duration of the scan. 


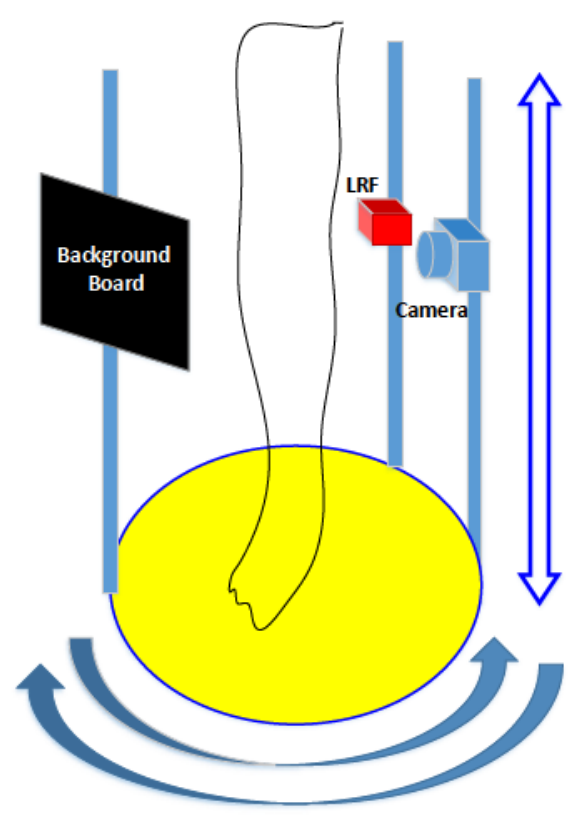

a.

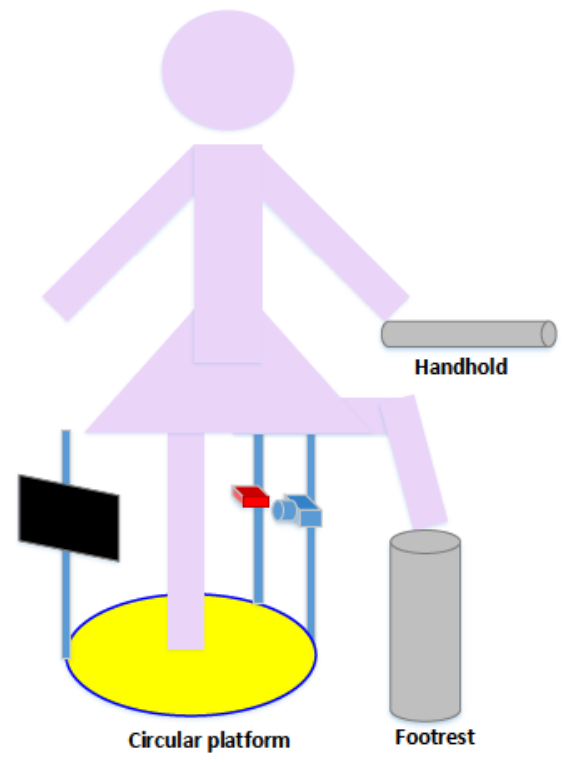

b.

Figure 3.1: System illustration: a. An illustration of the system assembly mechanism; camera (shown in blue), a laser range finder (shown in red) and a background board (shown in black) attached onto vertical rail-tracks; b. An illustration of patient during scanning procedure. The patient is holding handrails, one leg is placed on the footrest, while the other is being scanned.

The circular rail track consists of two subsystems: imaging and distance measurement. These subsystems are each mounted onto a vertical rail track. The vertical rail tracks are software controllable, thus they run horizontally on a circular rail track and they move the subsystems vertically for the whole length of a scanned leg. The movement of vertical tracks is synchronized, and performed in small steps, pausing for measurements and image capturing. 


\subsubsection{The imaging subsystem}

The imaging subsystem of the scanner consists of one digital camera and a background. The camera is mounted onto a vertical rail track; thus, it can move horizontally around the circular platform and vertically for the entire range of the leg's length. The camera points into the centre of the circular platform with the optical axis in parallel to the circular platform.

The requirements for the camera and the lens are:

- High resolution images - in order to detect lesion evolution by image comparison.

- Fast shooting speed - the system intent to shoot hundreds of images around the leg, necessitating high speed shots.

- Compatibility with macro photography - the platform has limitations on its size since the other leg needs a leg-rest, which means the camera is positioned close to the leg's surface.

- Computer interface for full control over camera parameters and image acquisition - to automate the intervals at which to take pictures of the patient.

- Price-quality ratio - overall system budget affects the frequency of the scans one can receive, and in order to catch malignant moles at their early stage frequent scans are necessary. Therefore, we are looking for a low cost camera with high quality resolution images.

The camera's internal parameters: F-stop, ISO value, exposure time, and autofocus [23] must be predefined for best image sharpness and for comparability of images 
through follow-up screenings.

One important requirement of resulting images is their sharpness. Image sharpness depends on camera's depth of field (DOF). DOF is the front to back zone where the image is maximally sharp and an object out of the DOF zone is out of focus. In our system settings, we want all the visible leg's skin to be in camera's DOF. A leg has a convex surface from the camera's viewpoint (the leg is approximately conical, thus, the centre of the captured skin image is closer to the camera, but the sides are farther). As such, we need to maximize the DOF to catch the fine details of the lesions. DOF depends on three factors: distance from the lens, focal length of the lens, and aperture size. While the former two are structural/equipment type settings, the last setting is controllable from the camera [23].

The aperture is the opening behind the lens that regulates the amount of light that travels through the lens and hits the image sensor. The size of the aperture's opening is measured in f-stops. F-stop works as inverse values, such that a small f/number (e.g. f/2.8) corresponds to a larger or wider aperture size, which results in a shallow DOF (with a larger aperture more light hit camera lens resulting more noise, therefore less sharpness) [23. To achieve deep images that are rich on details, a higher aperture opening preferred.

The camera's function of auto-focus should be disabled. Since the skin has a homogeneous texture, the camera has difficulty estimating focus distance by contrast detection used in auto-focus mode. Moreover, the scan is a time expensive procedure, and should therefore use the minimal time span for a single image, to minimize the full scanning time. Since we have chosen a deep DOF with the camera's aperture 
setting, the exact focus distance is less important for image sharpness, which should accommodate different leg complexions (thin or wide).

Shutter speed controls the amount of time for which the image sensor is exposed to light, represented as a fraction of a second. Long shutter speed lets more light enter and creates a better exposure for low light conditions, but it comes with its price: motion artifacts [23]. In our case, when making images of legs, slight muscle movements of the leg can create motion artifacts. Shutter speed should be optimized for low light condition, and reduction of motion artifacts.

ISO or light sensitivity rating defines image sensor's sensitivity to light [23]. The lower the number, the less sensitive the sensor is to light. Conversely, for low light conditions a higher number should be used. The lower ISO setting also means that the resulting image is smoother, since there is less digital noise in the image. ISO is one of the three elements that determine the final exposure of the photographic image, along with shutter speed and aperture setting. Lower ISO ratings produce colour-accurate, smooth and aesthetically appealing image, but this requires ideal light conditions. Therefore, for the best operation of our system, it should be placed in a well-lit room (ideally using daylight lamps), and the ISO setting should be selected as low as possible with respect to all aforementioned camera/room conditions.

When choosing a lens for the camera we need to minimize focus distance, since we are limited by the platform dimensions and compatible patient legs size variations. The platform radius is limited, since the second leg is lifted and placed on a leg-rest while the first leg is being scanned under it. Secondly, the platform radius defines the distance between camera and scanned skin, and a lens has a minimal distance to 
the object that has to be considered. All these aforementioned factors create a limit on the leg's dimensions. Therefore, in order to maximize compatible legs sizes, a lens that can focus from small distances should be used.

\subsubsection{Illumination}

An imaging system must also include illumination, as light is the most important factor for imaging with a digital photo camera. It is important to assume that the room used for the scanning procedure is a well-lit room, preferably with daylight lightning, such that the skin and lesions will be captured in colours close to their natural state. We will not go into further details about the lightning for our system.

\subsubsection{Background}

Any imaging subsystem is not complete without an appropriate background. In order to detect a patient's skin (image foreground) on an image, one can use complicated algorithms (for example the algorithm by Jones et al. [28]) or an appropriate background board which creates a good contrast with patient's skin and thus makes it an easy task to extract foreground from a background by contrast or by Hue-SaturationValue (HSV) (report on skin color detection techniques by Vezhnevets et al. [72]). This background board is mounted over another vertical rail track and is located $180^{\circ}$ from the camera's vertical track (Figure 3.1), behind the patient leg. The dimensions for this board are yet to be determined, as they depend on the platform size, and camera's zoom settings. 


\subsubsection{The distance measurement subsystem}

The distance measurement subsystem of the scanner consists of $1 D$ Laser Range Finder (LRF), which is mounted onto a vertical rail track such that it is pointed towards the centre of circular platform, in parallel with it. The vertical rail track with LRF is moving along a circular rail track around the platform and vertically along all the leg's length and collecting measured distances at specific stops. The purpose of this subsystem is to measure and collect distances to the skin from different locations, thus creating an accurate $3 D$ structure of the leg.

LRF measures the distance based on time of flight [5]. The emitter sends a laser pulse, while the receiver detects the reflected pulse from the surface. The electronics measure the round-trip time of the returning signal and its intensity. Since the speed of light in atmosphere is known, the system can deduce the distance to the surface. However, this technique is range-limited and accuracy-limited, since it depends on two factors: the minimal distance detectable by the electronics, and allowable power levels of laser radiation (from a safety standpoint).

The LRF constraints are:

- High accuracy in distance measurement (error below $2 \mathrm{~mm}$ )

- Class II, or I laser - no skin burn hazard on exposure

- Controllable through a computer interface (i.e., the data from LRF is sent to a computer for analysis and storage). 


\subsubsection{Vertical rail track subsystem}

In our system design we use three rigid vertical rail tracks in order to hold the camera, the background panel, and the LRF. The vertical rail track provides software controllable vertical movement for the attached device. These subsystems are mounted over a circular rail for a software controlled horizontal movement. Building the mechanics of these tracks is outside the scope of this research.

\subsection{The Scanning Procedure}

The scanning starting point is the lateral malleolus (Figure 3.2) - a bone which is located on the lower part of the leg and is visible in all leg types. The second reference point is medial malleolus, a bone located in the lower part of the leg on the inner side. The operator must mark a middle point (as accurate as possible) of the lateral and medial malleolus bones with red ink to set the origin and reference points of the leg respectively 1 1 . Then the operator must navigate the camera in-line with the marked point (vertically and horizontally) (Figures 3.3 and 3.4). That foot is placed on the platform in a position ensuring that the marked point on lateral malleolus (Figure 3.2 is directly above the marked line on the platform, and the heel aligns with the marked location on the platform. The other leg is lifted and placed on a foot rest. The patients must hold the hand holds provided for stability as the scanning process might take up to several minutes.

The camera and LRF have initial settings as well. The camera is positioned on

\footnotetext{
${ }^{1}$ Addressing the error of initial placement of the scanner was out of our research scope.
} 


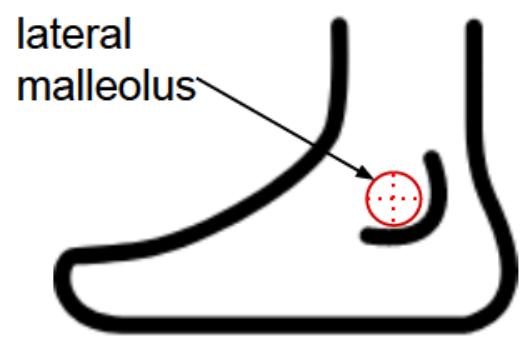

Figure 3.2: Diagram of how to locate the centre of lateral malleolus bone

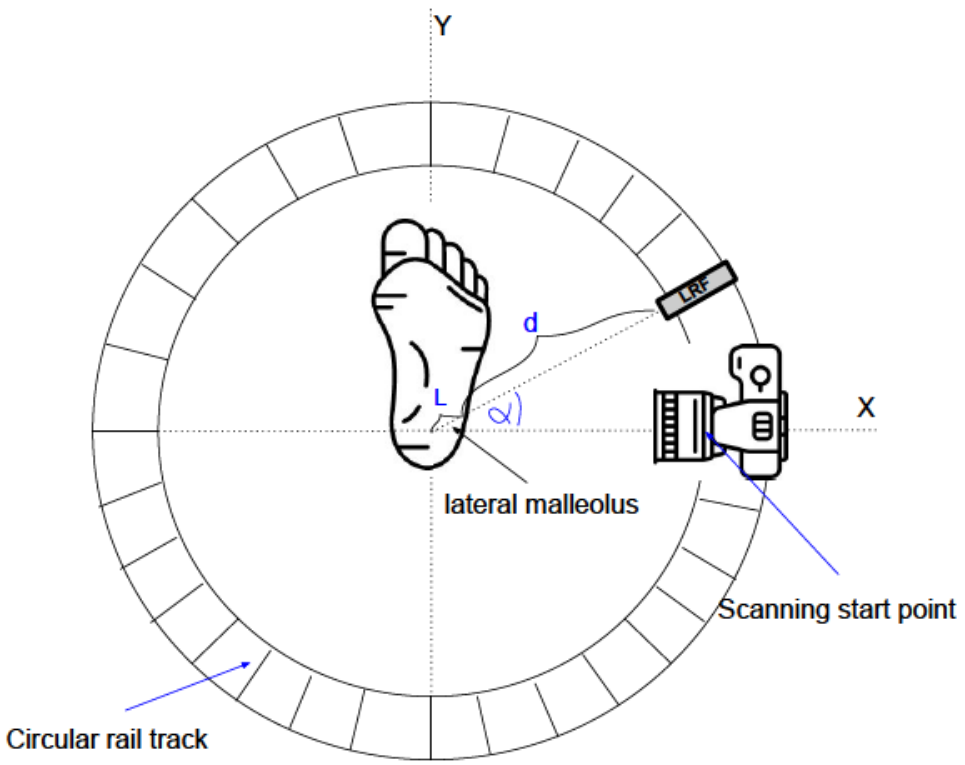

Figure 3.3: Platform top view 


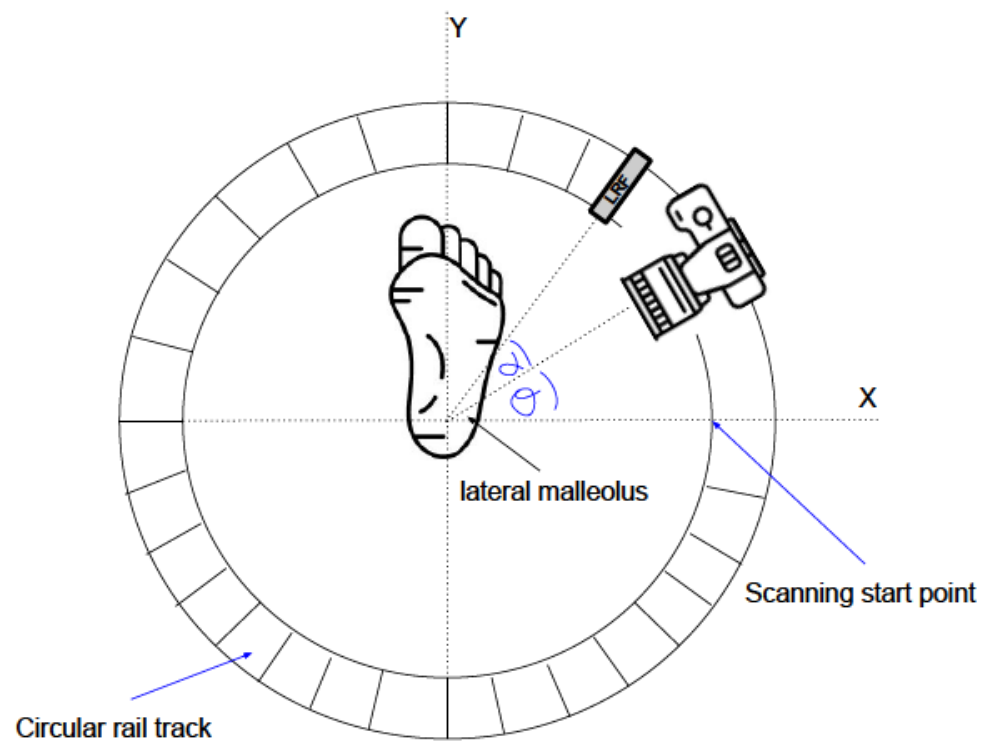

Figure 3.4: Platform top view after a move of angle $\theta$

the same vertical line as the centre of lateral malleolus bone. The background is $180^{\circ}$ apart from the camera, directly behind the lateral malleolus bone. The LRF and the camera are separated by constant smallest possible angle $\alpha$, defined by camera and LRF overall dimensions. The LRF and Camera's optical centres are located at the same height $H$.

During the scanning procedure, three vertical tracks move simultaneously on the circular rail, pausing for measurements in predefined intervals, $\theta$. After the completion of each full horizontal circle, the LRF, camera, and the background rise by a predefined distance on the vertical track. Once elevated, the vertical tracks continue to move along a horizontal circle, maintaining the same predefined horizontal angle interval $\alpha$. The system continues this behavior until the full height of the leg has been covered. At each aforementioned pause, the following information is collected: the camera 
takes an image of the leg, LRF registers the distance to the skin, and their positions within the system are recorded (height and angle with respect to starting point). The patient is required to stand still in order to receive an accurate correspondence between all collected information.

At each scanning point with angle $\theta_{i}^{L R F}$ (calculated from the systems' starting scanning point) and height $H_{j}$ from the floor, the LRF registers the distance, $d_{i j}$, from the the leg. The distance from the LRF to the centre of the platform, i.e., platform's radius, is denoted by $\rho$. Thus, the thickness of the leg from platform's centre at horizontal step $i$ and vertical cycle $j$ is

$$
L_{i j}=\rho-d_{i j}
$$

The camera's location is recorded in terms of the following parameters:

- Angle $\theta_{i}^{C}$ with $x$-axis

- Height $H_{j}$ from the ground

These data points, as well as the acquired images, are later used to create a $3 D$ mole map (Section 4.1.4.

\subsubsection{Definition of Coordinate Systems}

The world coordinate system is a right-hand system with its origin in the middle of a circular platform, where the $z$-axis is perpendicular to the platform. The $x$-axis is defined by two points - the centre of the platform and a point on the platform that 
is directly below the centre of lateral malleolus bone. The direction of the $x$-axis is defined by the right hand rule, which, in case of a right leg, is the direction from the centre towards projection of the lateral malleolus point onto the platform. The $y$-axis points toward the leg's toes, Figure 2.4.

Camera coordinate system is also right handed, where the $w$-axis coincides with the camera's optical axis from the camera centre towards the centre of the platform, and in parallel to it. The $v$-axis is directed downwards perpendicular to the platform, and the $u$-axis is directed to the right in parallel with the platform.

\subsection{System Limitations}

The envisioned system as described is intended for medical use, with a target of leg scanning for detection of newly appeared and changed lesions (which are signs of potential melanoma malignancy). With this consideration in mind, there are limitations defining the eligibility of the patient for scanning. Firstly, due to the nature of this system, the camera and LRF have to move around the scanned leg, with the second leg lifted above. Thus, a patient should be flexible enough to lift one leg, and stand still with the help of handholds for the entire duration of the scanning process.

Secondly, there are restrictions on the leg's shape (i.e., how wide the leg is), due to minimal distance requirement from the camera to skin, such that the image will be in camera's DOF. For example, if the minimal distance from the camera to the object in order to focus properly is $200 \mathrm{~mm}$, and platform's radius is $500 \mathrm{~mm}$, then the leg's width is limited by these two parameters (a leg with up to $300 \mathrm{~mm}$ in diameter will 
fit in this scenario).

Additionally, the system does not analyze the patient's movement in any way. It assumes that the patient is standing still for the whole duration of the scan. Patient movements can be separated to two sub categories: (1) a patient moves during a single shot is taken, (2) patients moves along the whole duration of the scan. In case of (1) category of movements, we would expect the image to be sharp enough for further analysis, since the selected aperture opening is small and shutter speed is fast enough to not let movements to create a big impact on the resulting image. However, in case of the (2) category of movements (i.e., small movements of the leg along scan duration), the resulted images would not correlate correctly with acquired LRF readings.

A possible way to analyze patients' movements is a use of additional static LRF on the perimeter of the leg (for example four or six additional LRFs) at the upper part. The additional LRFs will check for leg's movements and either stop the scanning process until the leg returns to its original position or calculate the movement distance and fix the LRF measurement at the scanning point by extrapolation of error due to movement to the scanning point location.

\subsection{Conclusions}

We presented an innovative approach of $360^{\circ}$ leg scanning using LRF and digital camera for the detection of malignant melanoma moles. The system is automatic and the operator's involvement is minimal - marking the initial point on the lateral malleolus 
bone, and helping the patient to stand on the marked place on the platform. The system returns high resolution overlapping digital images coupled with the distance to the skin and camera/LRF locations in world coordinates.

The scanner provides a controlled environment due to the predictable starting point, intrinsic calibration of the camera, and the overall system structure - where the LRF's and camera's optical centres coincide on a perpendicular line to a platform originated at the centre of it. The distance between camera and LRF is known. Thus, it allows lesion registration between sequential scans.

We presented an envisioned system design. However, the exact details can only be determined when constructing a prototype and testing it on real patients. There is a need to choose the digital camera, lens, and LRF that are the most suitable for this scanner. The exact measurements of the system are yet to be decided, such as circular platform radius, vertical track length, as well as the design of the vertical and horizontal rail track is yet to be determined. When selecting a focus point of the camera during the system calibration and setup, different body types should be taken into account since the initial focus point has an impact on the DOF for all patients.

In order to speed up the process of leg scanning, it is possible to add more cameras and LRF to the scanner placed in vertical row one on top of each other. Thus, the scanning time will be divided by number of camera-LRF pairs were added. This optimization creates a trade-off between system cost versa scanning time.

One of the system requirements we set here is low cost. Low cost system means better availability, resulting in more frequent scans for the patient, hence, catching 
evolving moles early. A low cost semi-automatic system can be installed in a community clinic, and it does not require a physician attendant.

Moreover, our system could be suitable for tele-medicine: when a physician is physically located hundreds of miles away from their patient and still can provide medical care. A physician can look at acquired images and check for visible signs of melanoma, and raise concern if needed. With some software tweaks, the medical professional could manipulate camera's location around patient's leg real-time, and thus have a better view of concerning moles while not being in the same room with the patient. It is especially important in countries like Canada, since it is a large country, with remote areas where there is no access to a doctor with narrow specialties yearround. 


\section{Chapter 4}

\section{Methodology}

The skin scanner system outputs a sequence of images and LRF readings, each accompanied with spatial orientation and position. The LRF readings are used to create a $3 D$ model of the leg, and the images are used for nevi identification. The $3 D$ leg model and images are combined to create a $3 D$ mole map. The resulting mole map is used as a baseline for subsequent scans. The subsequent mole map (from a followup scan) is compared with the baseline mole map for nevi registration and change detection (Figure 4.1).

In this chapter we describe our algorithm to process data from a single scan in order to create an intra-exploration mole map (Section 4.1), and to automatically match a subsequent scan to a baseline scan for the creation of temporal scan exploration mole correspondences (Section 4.2). 


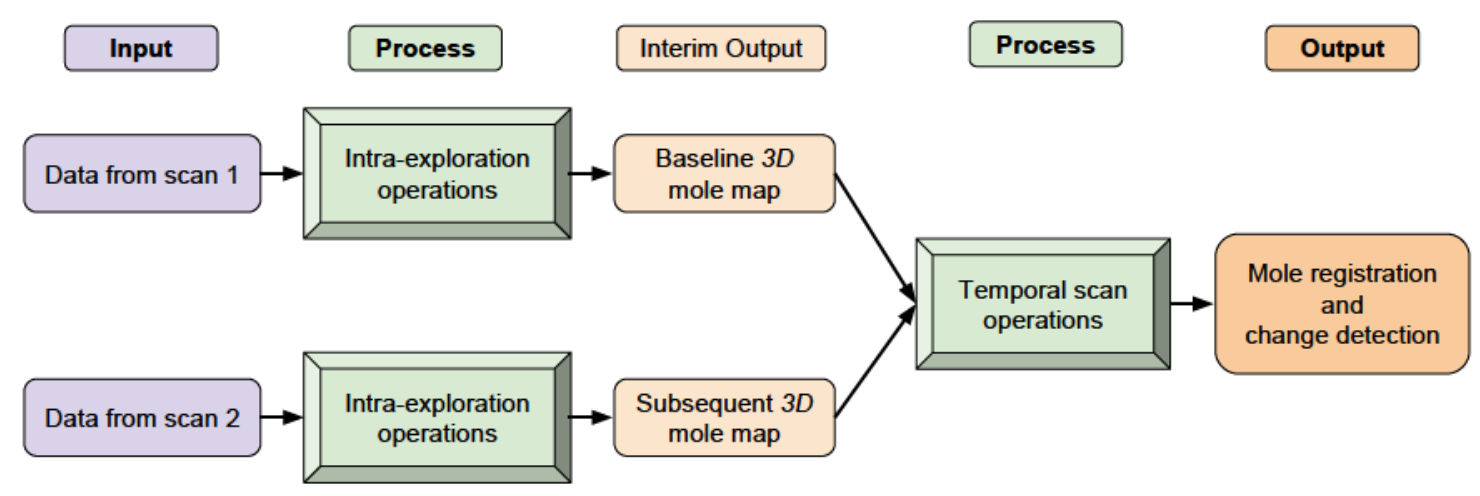

Figure 4.1: The process for mole registration and change detection through temporal scans.

\subsection{Intra-exploration Mole Mapping}

The intra-exploration mole mapping process combines LRF readings and camera images and synthesizing them into a $3 D$ leg model with PSLs locations. The input data of this process is:

- LRF readings coupled with location of LRF

- Images of the leg coupled with location of camera

Camera and LRF locations are denoted by angle from $x$-axis, $\theta^{C}$ and $\theta^{L R F}$, respectively. Their vertical locations are equal and denoted by $H_{j}$, where $j$ is the index full horizontal cycle, further referred as layer.

The scanner frequency, i.e. the horizontal step size of camera and LRF, are denoted by $\Delta C$ and $\Delta L$, respectively, and the vertical step size is denoted by $\Delta V$. These parameters are set so that images taken from two adjacent locations horizontally or 
vertically have an overlap of at least $50 \%$. Thus, we ensure that each mole will appear on at least two adjacent (horizontally or vertically) images. Each instance of a mole on an image is referred to as a mole view. The algorithm matches mole views on adjacent image pairs in order to record each mole only once. Adjacent image pairs are divided into two groups:

- Horizontal - two images taken from two consecutive angles, $\theta_{i}^{C}$ and $\theta_{(i+1)}^{C} \bmod n$, while the layer $H_{j}$ is held constant. Here $n$ denotes number of images in one layer.

- Vertical - two images taken from the same angle, $\theta_{i}^{C}$, from two consecutive layers $H_{j}$ and $H_{j+1}$.

Since the views are taken from different angles and heights, a particular PSL can be represented better in some views than in other views. Due to the nature of the leg's shape it is safe to assume that the optimal mole view, or the least distorted view is one where the mole's center is closest to the optical centre of the camera. In other words, the optimal view representing the mole is where it is closest to the image centre.

The process of intra-exploration mole mapping consists of four main steps (Figure 4.2):

1. Mole location. For each mole view we locate four closest LRF readings (after projecting them onto the image plane), Section 4.1.1.

2. Mole pairs. We match mole views on all adjacent (horizontally or vertically) images to create mole pairs, Section 4.1 .2 
3. Mole sets. A mole set consists of all the views (taken from different heights and angles) representing a single mole. Mole sets are generated from linking mole pairs, Section 4.1 .3 .

4. $3 D$ mole map. We select an optimal mole view of each mole from a mole set. These moles are mapped onto a $3 D$ leg model by their relative location with respect to three surrounding LRF points, Section 4.1.4 

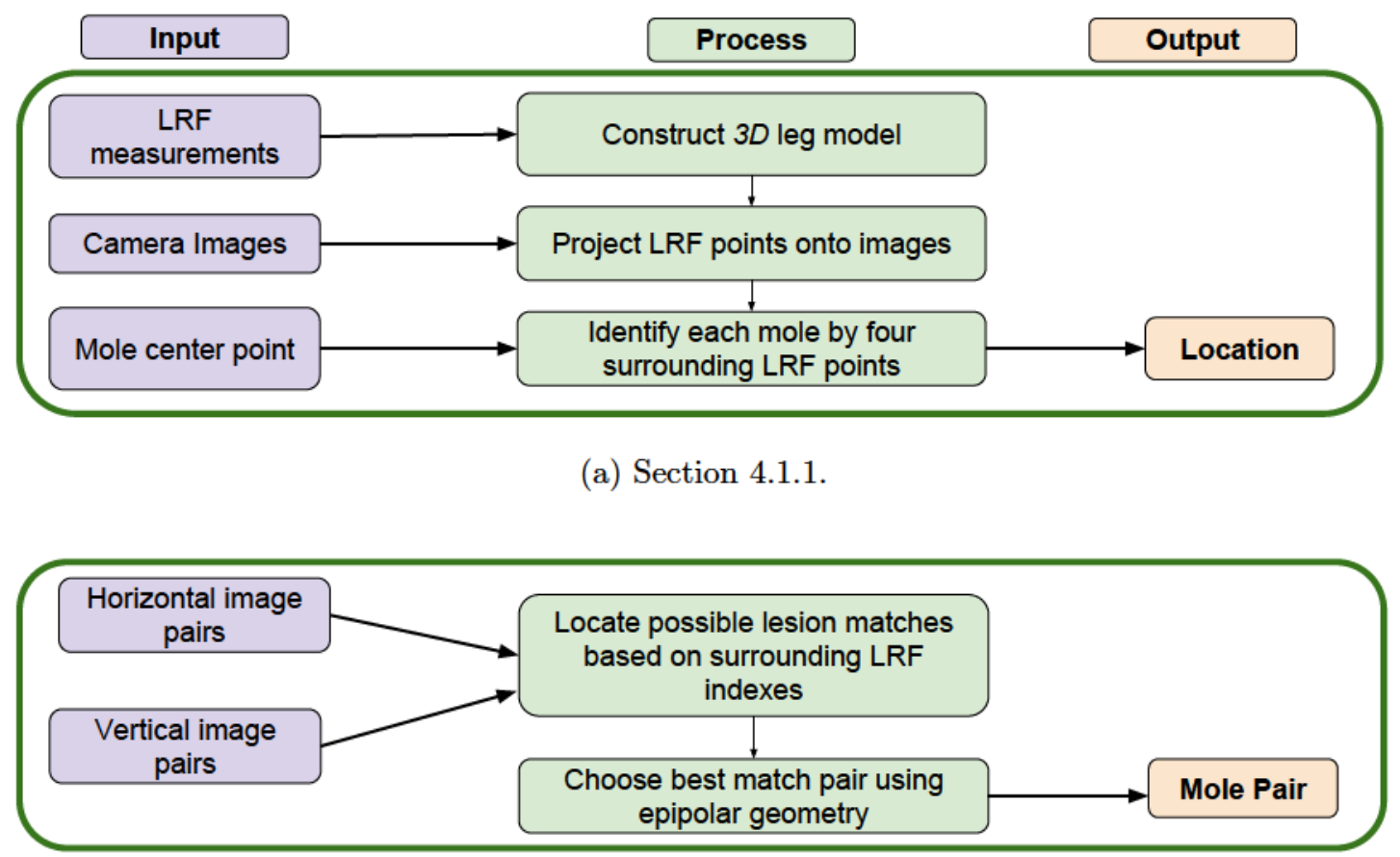

(b) Section 4.1 .2 .

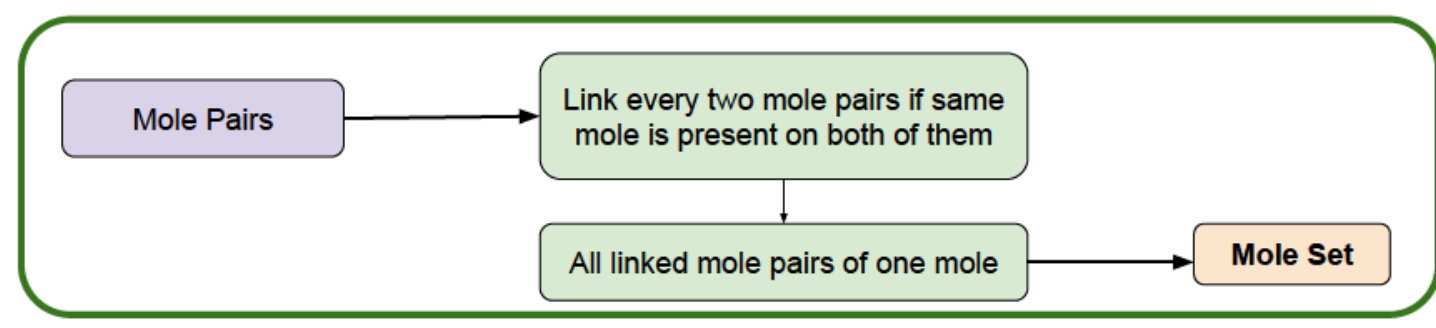

(c) Section 4.1.3.

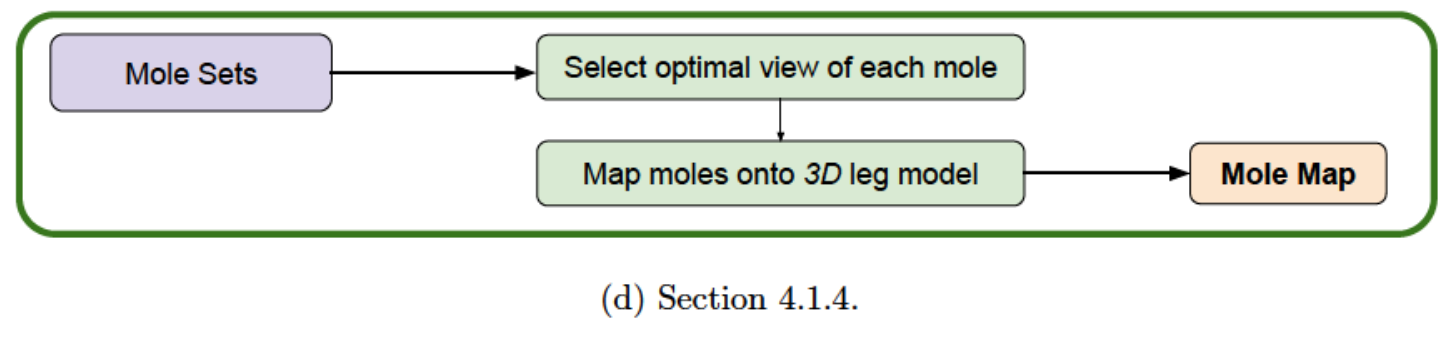

Figure 4.2: Intra-exploration mole mapping process flow 


\subsubsection{Mole Location}

In this step of the algorithm we link each mole view to the four closest surrounding LRF readings. First, we create a $3 D$ leg model in world coordinates from LRF readings. Then, we project these $3 D$ leg model points onto the images obtained from the pinhole camera model described in Section 2.4.1. Later in our algorithm we use these surrounding LRF readings for the creation of mole pairs (Section 4.1.2) and the mapping of moles onto a $3 D$ leg model (Section 4.1.4).

The LRF readings are captured in a systematic way: the LRF moves around the platform taking measurement with step intervals $\Delta L$. After the completion of a full cycle, it raises vertically to the next layer (Section 3.2 ). Let $d_{i j}$ denote the $i$-th LRF reading at layer $j$. The thickness of the leg, denoted by $L_{i j}$ at this location is computed by Equation 3.1 .

The location of the LRF at that measurement is defined by height, $H_{j}$, and angle, $\theta_{i}$. Thus, the point, $\boldsymbol{r}_{i j}=\left(x_{i j}, y_{i j}, z_{j}\right)$, in system coordinates, is denoted by Equation 4.1. The origin, in system coordinates, is at the centre of the circular platform. The $3 D$ leg model is created from a set of all LRF points $\boldsymbol{r}_{i j} \in \mathcal{R}$.

$$
\begin{aligned}
x_{i j} & =L_{i j} \cos \left(\theta_{i}^{L R F}\right) \\
y_{i j} & =L_{i j} \sin \left(\theta_{i}^{L R F}\right) \\
z_{j} & =H_{j}
\end{aligned}
$$

Each $3 D$ world point (Figure 4.5 (a)) of LRF readings is projected onto the image 


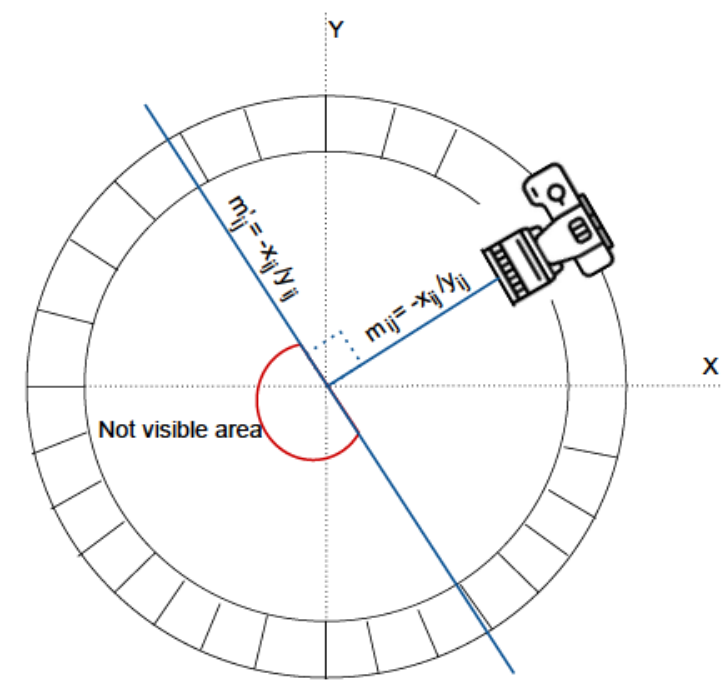

Figure 4.3: Platform top view - visible part of the leg

taken by the camera, using Algorithm 1. The resulting camera images contain mole centers and LRF points (Figure 4.5 (b)).

The LRF world points provide $3 D$ information about the leg, but only half (or fewer) of them are visible to the camera at any location (Figure 4.5 (c)), since the leg is an opaque object. In order to check which points are visible from the camera's location, we calculate a cut-off plane of visible points (Figure 4.3). First, we calculate the slope on of the $X-Y$ plane between camera located at $\left(x_{i j}, y_{i j}, h_{j}\right)$ and platform's centre, $m_{i j}=y_{i j} / x_{i j}$. Then we calculate a perpendicular slope, $m_{i j}^{\prime}=-1 / m_{i j}$. This plane is going through the centre of the platform. Thus the cut-off plane equation is: $m_{i j}^{\prime} x+y+0 z=0$. Only points that are on the camera's side of this line are visible to it. An exception occurs when the camera coincides with the $x$-axis or $y$-axis, in which cases the cut-off plane is $0 x+y+0 z=0$ or $x+0 y+0 z=0$ respectively.

The LRF projected points' information is coupled with an index pair $(i, j)$, that 


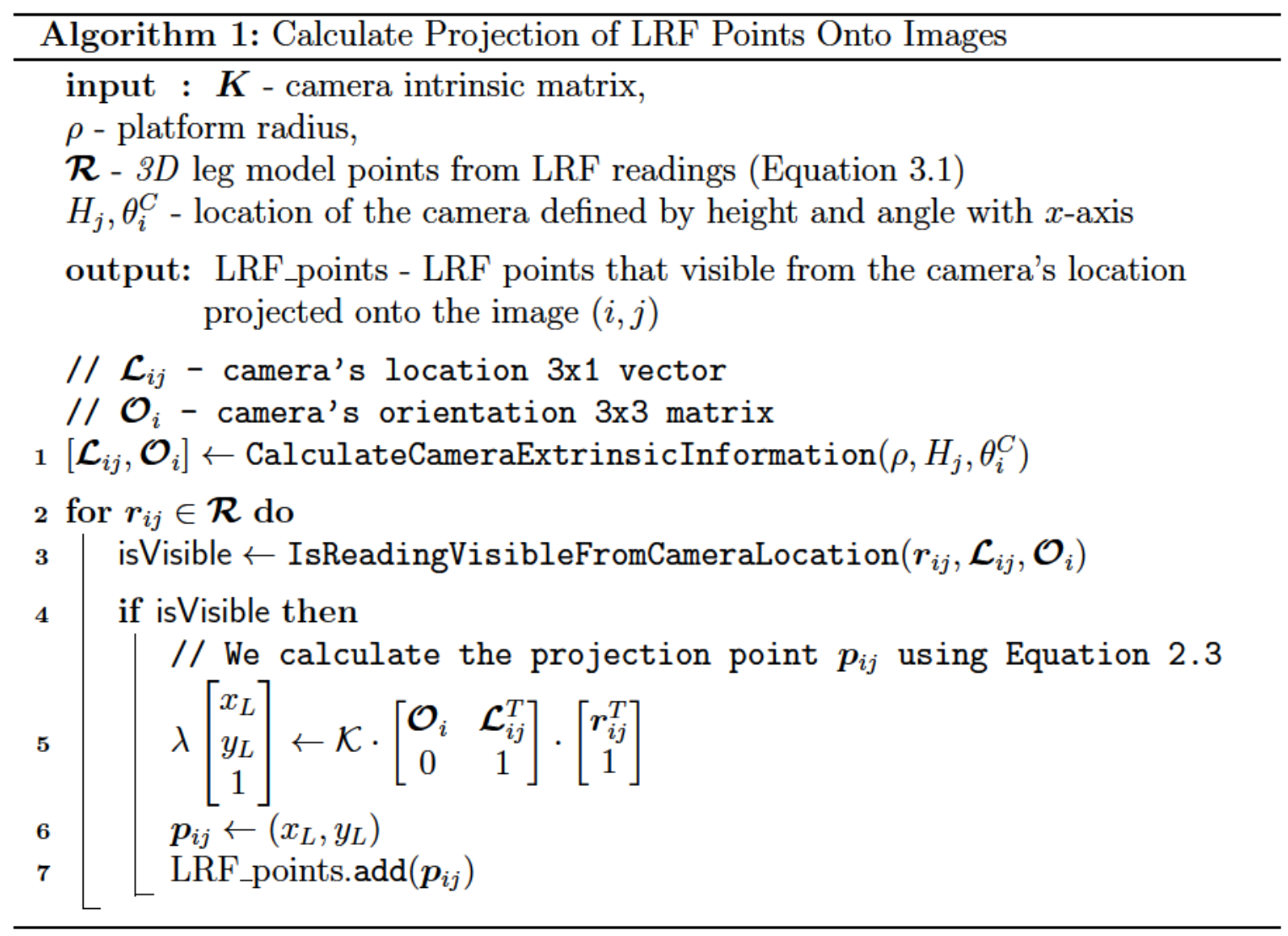


represents the horizontal and vertical positions, respectively at measurement time. This index system starts with a measurement taken from the angle $\theta_{i}^{L R F}=\alpha$ and $\theta_{i}^{C}=0$, where the $x$-axis coincides with the centre of the lateral malleolus and the optical centre of the camera (the origin of coordinate system was explained in Section 3.2.1.

Mole location is a process of identification of four LRF points (two points from upper row, and two points from lower row) for each PSL where the PSL is captured in the camera's view. This is done in two steps: first, we find the closest LRF point (as described in Algorithm 2), and then we locate three more points, thus receiving a quadrilateral around the PSL's centre:

1. We surround the image points with phantom points, ten on each side 1 .

2. We construct a Euclidean Voronoi diagram 2 from all the LRF and the phantom points on the $2 D$ images with LRF projections [7]. Then, for each mole $p$, we identify the Voronoi cell containing $p$, thus associating mole $p$ and closest LRF point (Figure 4.4).

We chose the Voronoi diagram algorithm because it provides a partition of the whole image. Thus, we can locate the closest LRF point for all PSLs in the image in one operation with a complexity of $O(n \log n+n m)$ compared to $O\left(n^{2}+n m\right)$, where $n$ denotes the number of LRF points on the image and $m$ denotes the number of moles on the image.

\footnotetext{
${ }^{1}$ By surrounding LRF points with phantom points, we make sure that all LRF points are defined by limited area Voroni regions, since the regions on the outer edges of Voronoi diagram end at infinity.

${ }^{2}$ Voronoi diagram is a partition of a plane into regions (Voronoi cells) surrounding points. A voronoi region defines an area where any point in the plane is closest to the regional seed, as compared with any other point.
} 
3. We locate three additional LRF points that surround mole $p$. Thus, we end up with four LRF points (right top, left top, right bottom, and left bottom) around a mole in the image.

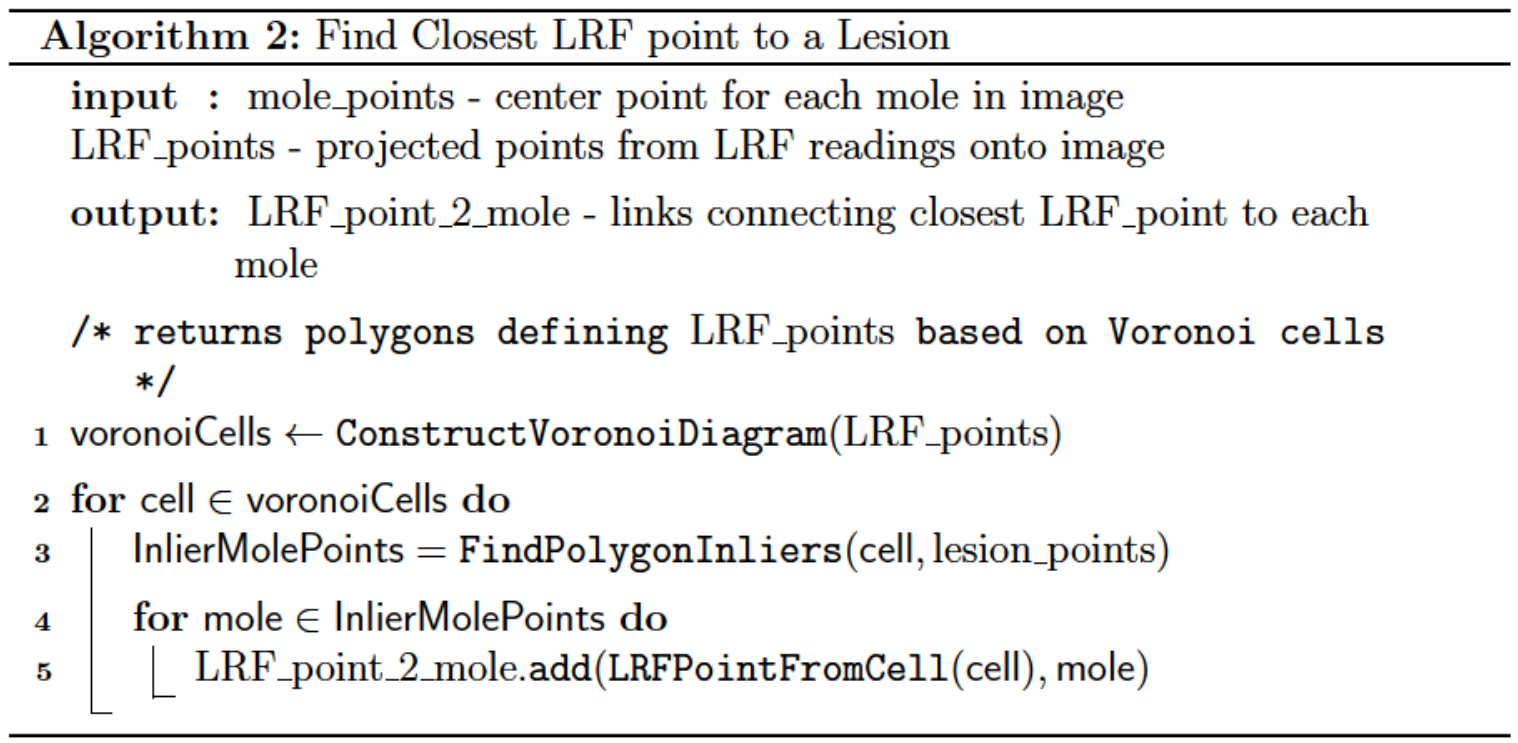

\subsubsection{Region of Mole Distortion}

The PSLs that are closer to the camera's optical centre are better represented than those which are close to the edges of the image. The convex shape of the leg produces a non-linear projection. The region closer to the optical centre of the camera has small curvature, so its projection can be seen as a plane (i.e., the distortion is negligible). However, regions farther away from the optical centre have high curvature, which generates a more distorted projection. Therefore, we define a region of mole distortion, such that moles from that region are considered to be distorted and irrelevant for further consideration. 


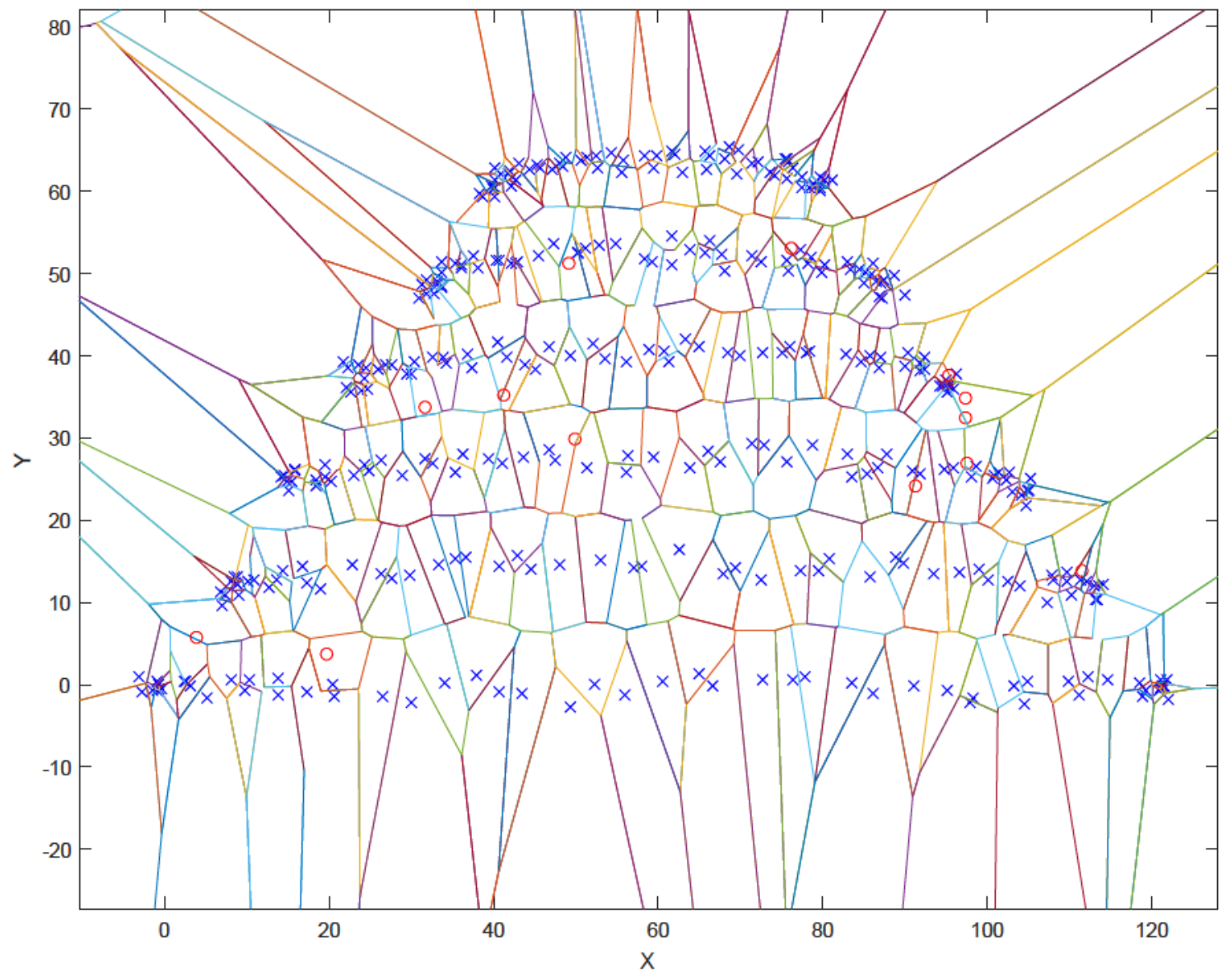

Figure 4.4: Voronoi cells (colourful lines) around LRF points (blue ' $x$ '), and location of moles (red 'o'). Voronoi diagram allows to locate the closest LRF point to each mole in a computationally effective manner. 


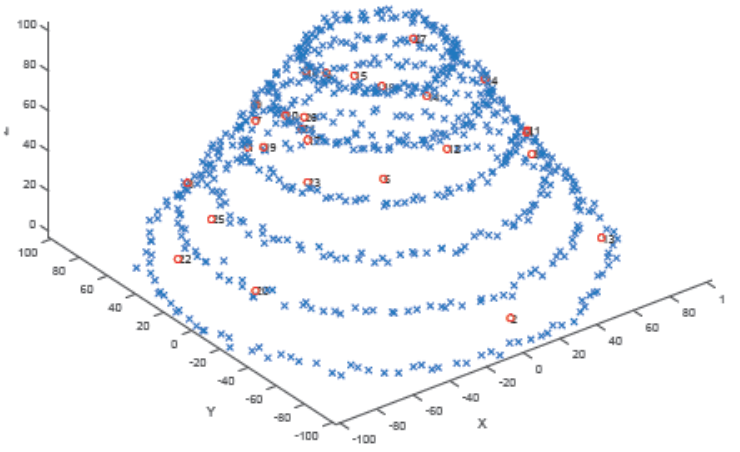

(a) $3 D$ Leg Model

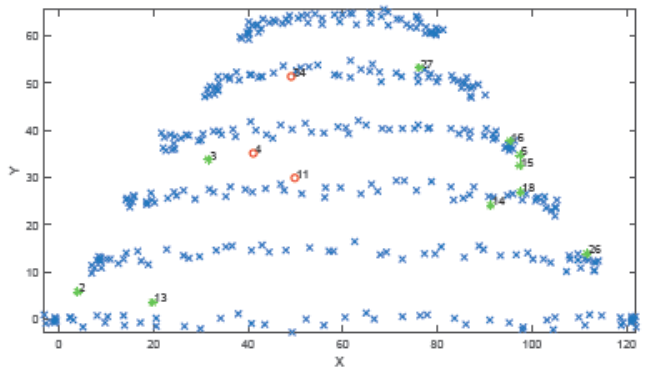

(b) Image formed from the $3 D$ leg model by pinhole camera formalism. The ' $x$ ' represent LRF points, 'o' - moles outside of region of moles distortion, '*' - irrelevant moles as they are inside the region of moles destortion

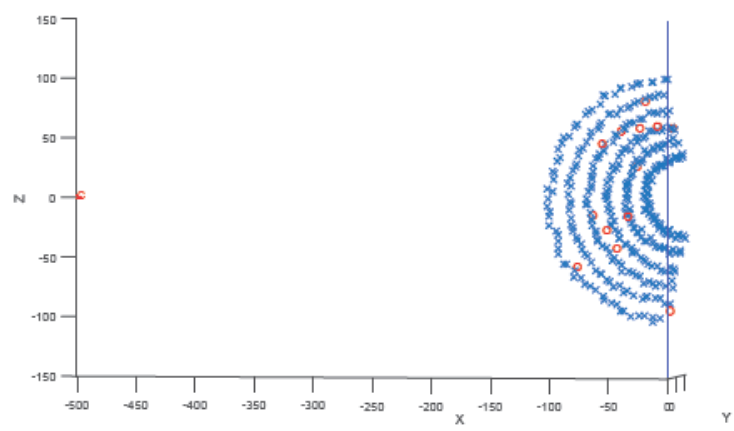

(c) Visible LRF points and PSLs from a camera (in camera's coordinate system) at 0 location $(\theta=$ $0, h=0, x=-500)$

Figure 4.5: $3 D$ leg model and image formation. Visible LRF points on the conical frustum are projected into image, as well as PSLs based on pin-hole camera model and form a $2 D$ image. The ' $x$ '-s represent LRF readings and the 'o'-s represent centre locations of the PSLs 


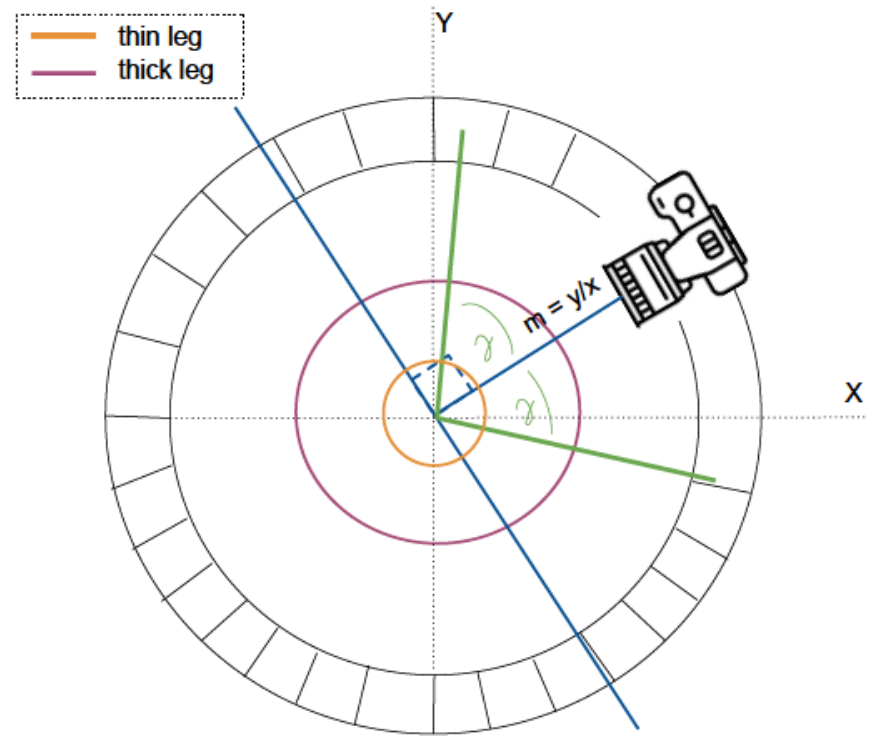

Figure 4.6: Platform top view - region of relevant PSLs is defined by angle $\gamma$ from optical centre. Two cases of various legs radiuses are shown

In order to determine which moles are relevant and which are distorted we define an angle of relevance, $\gamma$, from the optical centre (Figure 4.6). Thus, moles that are located angle $\gamma$ or less from the optical centre are relevant, and those located outside of that region are distorted and irrelevant.

Although an angle of relevance depends on a leg's thickness, i.e., for a thick leg the suitable angle $\gamma$ is bigger than for a thin leg (Figure 4.6), we decided to use one angle limit for all leg types and leg's areas in this research.

In each image, in order to extract relevant PSLs out of all moles, we check their corresponding four surrounding LRF points. If all four LRF points of a mole are inside the region of mole distortion then a mole is marked as irrelevant. Further along in the chapter, we discuss only to moles that are not marked as distorted. 


\subsubsection{Mole Pairs}

From our system's definition we ensure that each mole has multiple views: we chose horizontal and vertical intervals for taking images such that every horizontal/vertical pair has at least $50 \%$ of shared skin region. A leg's shape (assuming that legs are convex) has no occluding contours, i.e., the mole $p$ appears on a series of consecutive images, until it is not visible anymore to the circulating camera. The mole $p$ also appears on images from two adjacent levels. Thus, we need to pair views of the same PSL on adjacent images (Section 4.1).

Mole paring is done in two steps:

- First we create tentative pairs by comparing four surrounding LRF readings. For each mole pair from two adjacent images, if the difference between surrounding LRF indices is within a tolerance distance (we used difference of three LRF indices), the moles are marked as a tentative pair.

- We confirm the match using photogrammetry (Section 2.4.2). A fundamental matrix is computed between every two adjacent images. In order to compute a fundamental matrix, we take LRF points on the images for the matching features. We use a fundamental matrix function implementation by P. Kovesi [33] that requires eight or more matching points in stereo images. Then we compute an epipolar line from the mole's centre point on the second image and choose one of the moles whose centre has a minimal distance to the epipolar line. We mark that PSL as a match, and do not consider it for further matches. Empirically, we chose $2 \mathrm{~mm}$ as the maximally suitable distance from the mole's center to the epipolar line. 


\subsubsection{Mole Sets}

After mole pairs have been identified on horizontally/vertically adjacent images, we have multiple representations of the same PSL linked together. These pairs are grouped into mole sets. A mole set consists of all the views representing the same mole across the images.

In order to organize the PSL views into mole sets, the matched moles are registered with a doubly linked list. For example, a mole $M_{i, j}^{a}$, which is mole $a$ on image $(i, j)$ is linked to mole $M_{(i+1) \bmod n, j}^{b}$ which is horizontally adjacent, and to mole $M_{i, j+1}^{c}$ which is vertically adjacent. Mole $M_{(i+1) \bmod n, j}^{b}$ is also linked to horizontally adjacent mole $M_{(i+2) \bmod n, j}^{d}$ and vertically adjacent mole $M_{(i+1) \bmod n, j+1}^{e}$. This results in five views representing the same mole on the patient's body: $M_{i, j}^{a}, M_{(i+1) \bmod n, j}^{b}, M_{i, j+1}^{c}$, $M_{(i+2) \bmod n, j}^{d}, M_{(i+1) \bmod n, j+1}^{e}$, where $n$ denotes number of images in one layer.

\subsubsection{Limitations of Mole Sets Creations}

In rare scenarios mole sets were not created correctly. These scenarios are divided into two groups:

1. A single mole can be represented as more than one PSL, i.e., during creation of mole pairs, a link between two views of the same mole was not established due various reasons (for example, if leg movement occurred and the views did not match epipolar lines), resulting in two or more mole sets for the same PSL.

2. One mole set contains views of more than one mole, i.e., two different moles were mistakenly paired together (for example if the two moles are very close to 
each other).

This research did not include a method to overcome these scenarios. We recommend exploring homographical transformation (a small to medium mole and its close surroundings can be considered as if located on plane) and comparison of resulting mole views for additional confirmation of mole matching.

\subsubsection{D Mole Map}

After mole sets have been identified, we select the optimal view representation of the mole. Due to leg geometry resembling a conical frustum, the optimal view of a mole is a view where the PLS's centre is closest to the image centre. In this case, the view is least warped. Therefore, for each mole representation we calculate the distance from its centre to image centre and select the view with minimal distance.

Optimal views of all moles are combined into an optimal view mole list. The list contains all identified PSLs and their views with four surrounding LRF points. That information in $2 D$ is used to map moles onto a $3 D$ leg model as follows:

For the identification of the relative location of mole centre, $l_{C}$, we take three LRF points: top right, $t_{R}$, top left, $t_{L}$, and bottom left, $b_{L}$. We connect these LRF points with lines: top right and top left points with a line segment $L_{R L}(s)=>t_{L}+s\left(t_{R}-t_{L}\right)$; bottom left with top left $L_{T B}(s)=>b_{L}+s\left(t_{L}-b_{L}\right)$ (Figure 4.7 for a schematic view). Next, we project the PSL into $L_{R L}$ and $L_{T B}$ The distance between the top LRF points is $D_{1}$, and the distance between the two left LRF points is $D_{2}$. The distances from the PSL to the top and the left lines are denoted as $d_{1}$ and $d_{2}$, respectively. 


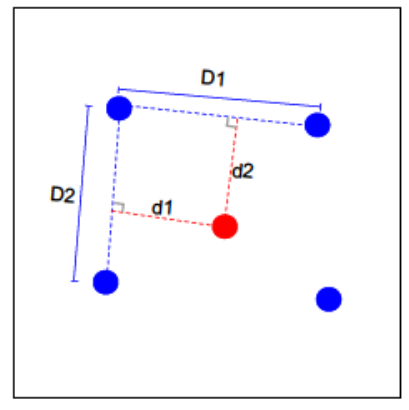

(a)

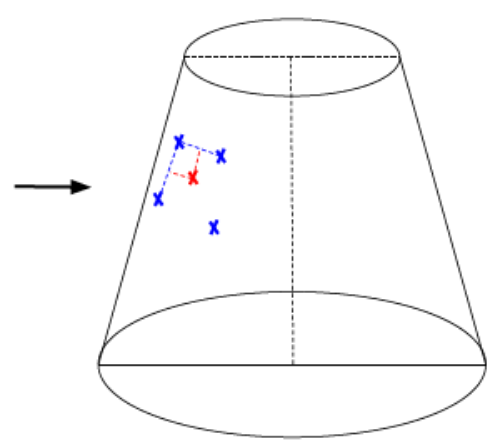

(b)

Figure 4.7: (a) Schematic representation of mole location (red dot) with relation to surrounding four LRF points (blue dots), and how we measure relative location of the mole on a $2 D$ image. (b) Projection of a 2D mole location with respect to surrounding LRF points onto a $3 D$ leg model.

We calculate the relative PSL distance as follows: the relative horizontal and vertical

distances, respectively, are $D_{R 1}=\frac{d_{1}}{D_{1}}$ and $D_{R 2}=\frac{d_{2}}{D_{2}}$. These relations are used to place the PSL on a $3 D$ model in a reversed manner.

These calculations do not result in the exact original location of the moles, but they give a very close approximation which will help register mole matches (Section 4.2 for temporal scan exploration operations). An example of a $3 D$ leg and subsequent map as calculated by the described algorithm in this chapter is shown in Figure 4.8.

\subsection{Temporal Scan Operations}

Temporal scan PSL registration is a process of matching moles from two scans taken with time interval. During this time period, some moles could have changed (in size, colour, texture), some could have disappeared, and new moles could have appeared. 


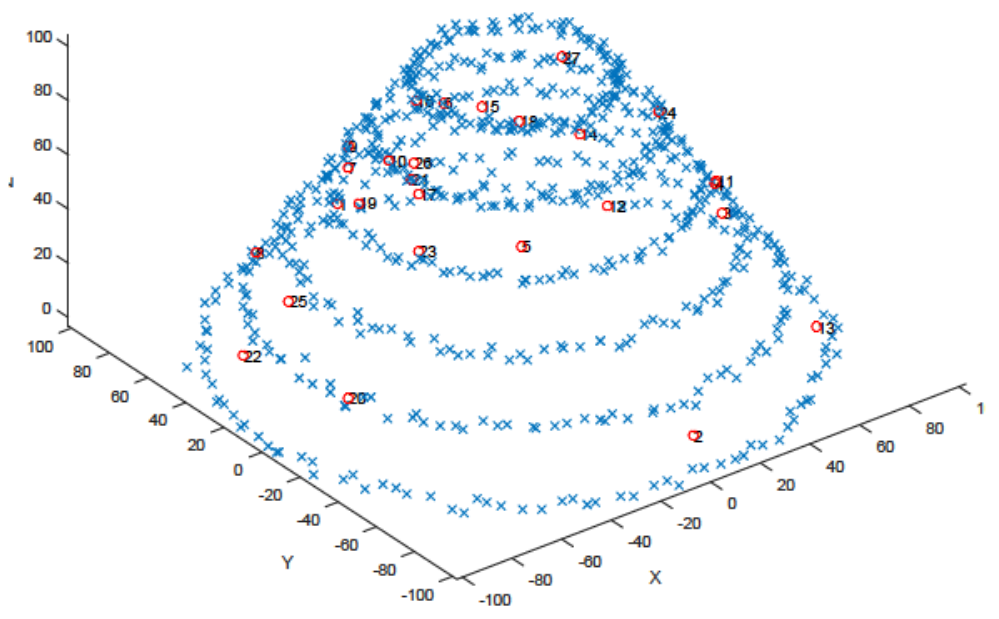

(a) Simulated leg

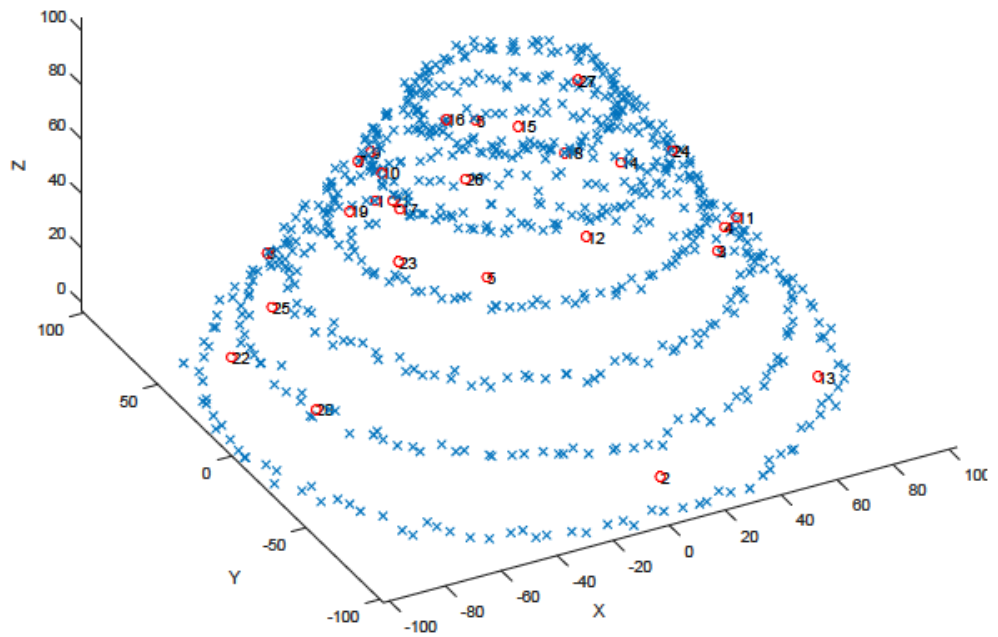

(b) Computer $3 D$ model

Figure 4.8: Comparison between original simulated leg model, and calculated mole map. Lesions are identified by their ID number. ' $\mathrm{x}$ '-s represent LRF points and 'o' represent moles. The average distance error and standard deviation in this simulation is $2.089 \pm 0.275 \mathrm{~mm}$. 
The purpose of mole registration is to identify those changes. Lesions that transform over time, or newly appearing ones, are a strong indicator of potential malignancy, and should be accurately examined by a dermatologist.

The process of mole registration from two temporal scans involves additional complications apart from matching moles that have transformed:

- During this time interval the leg could change in size and shape (e.g. due to muscle growth, water accumulation).

- Initial scanning point could slightly move; thus the result leg model would not match exactly to the original model $3 D$ locations. This case is out of our research scope.

- The leg position itself varies across temporal scans. This case is out of our research scope.

For finding correspondence between two mole maps we decided to use the CPD algorithm by Myronenko et al. [47, 48]. This algorithm finds correspondences between two point sets (in $2 D$ and $3 D$ ), overcoming the presence of outliers and missing points (described earlier in 2.4.3). 


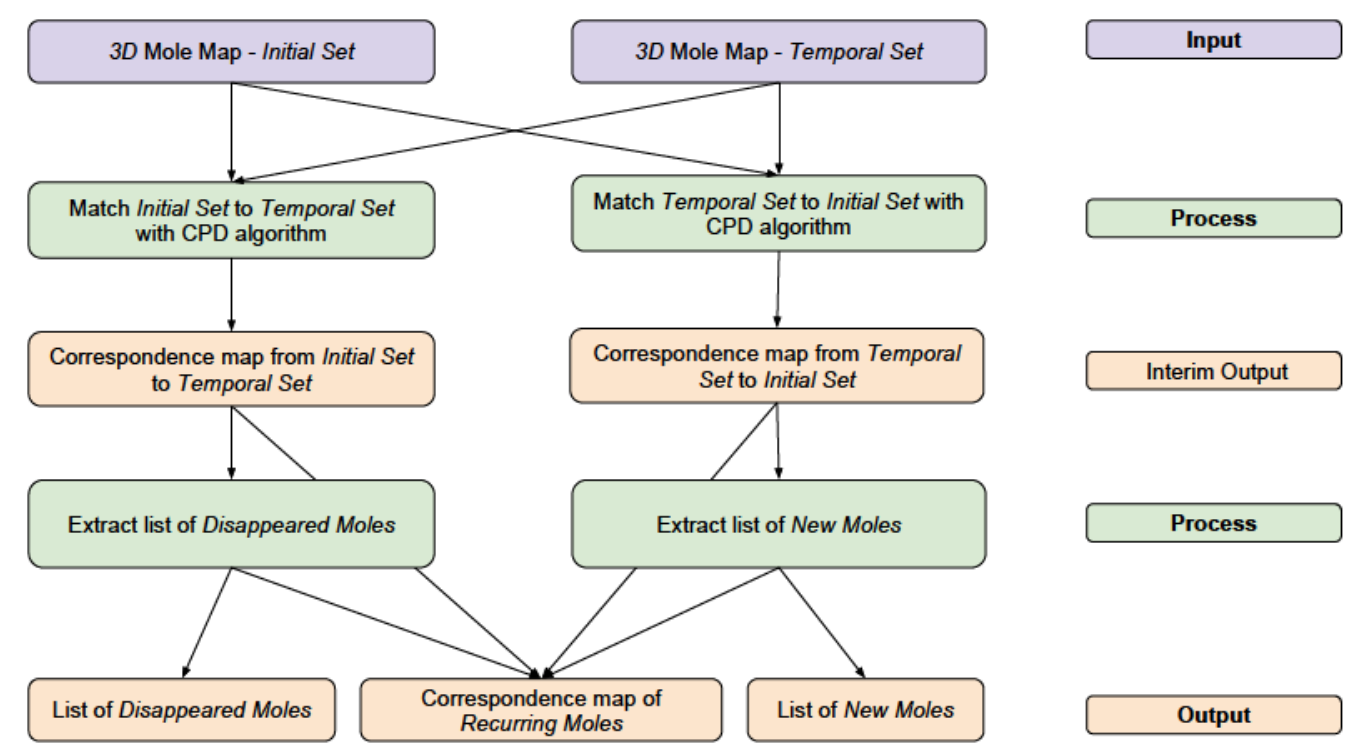

Figure 4.9: Overview on temporal scan operations process

\subsubsection{Match by CPD Algorithm}

The input data for temporal scan operations is two sets $\boldsymbol{S}_{\boldsymbol{t}}$ and $\boldsymbol{S}_{\boldsymbol{t}^{\prime}}$ of $3 D$ points acquired from two scans at times $t$ and $t^{\prime}$, where $t<t^{\prime}$. From these sets, first we find deleted moles set $S_{D}$ by applying Algorithm 3 . Then, we find new moles set $S_{N}$ by applying Algorithm 4. Finally, we apply Algorithm 5 to find correspondence map of recurring moles from set $S_{t}$ to set $S_{t^{\prime}}$. This concludes temporal scan operations. 

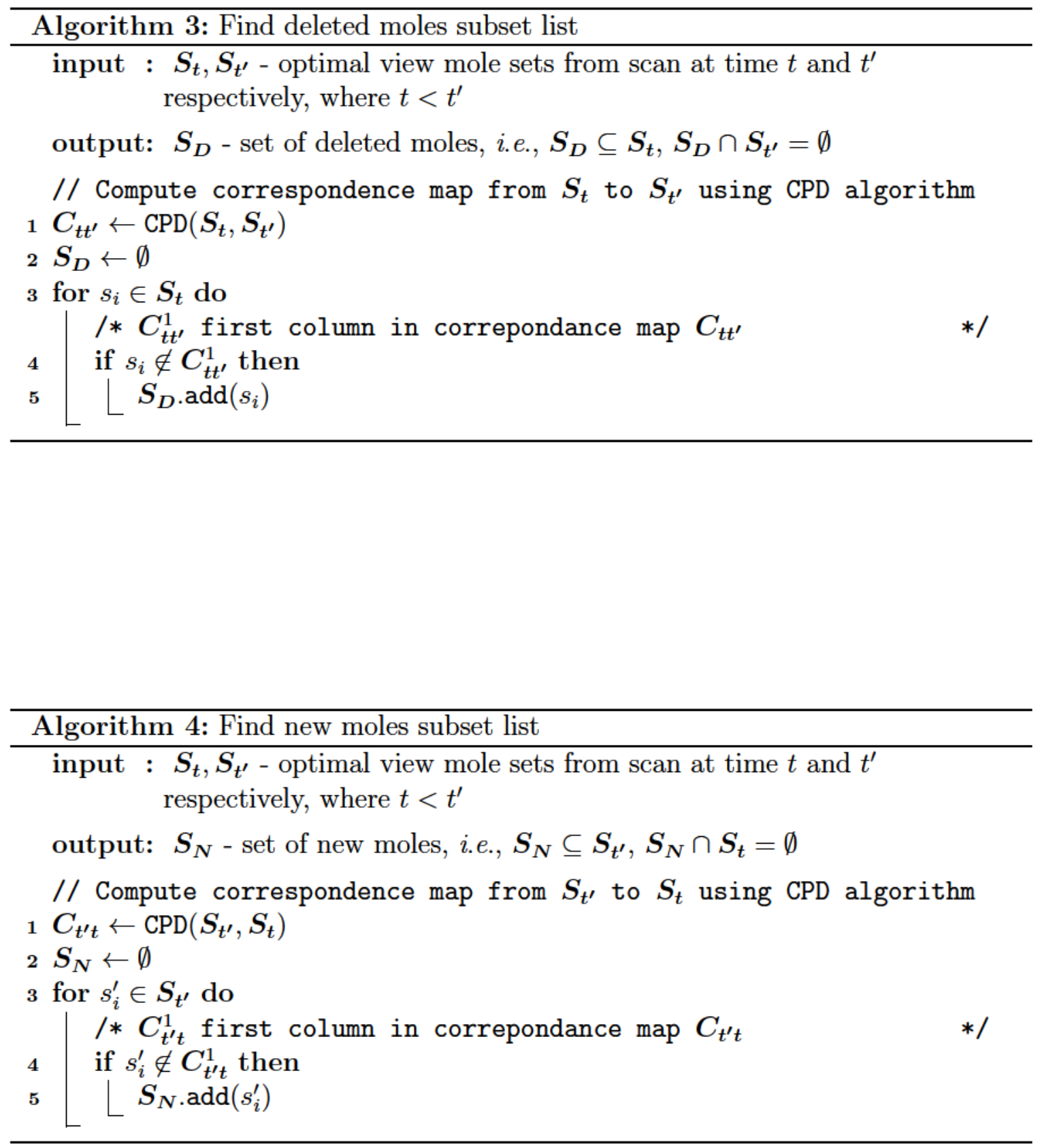


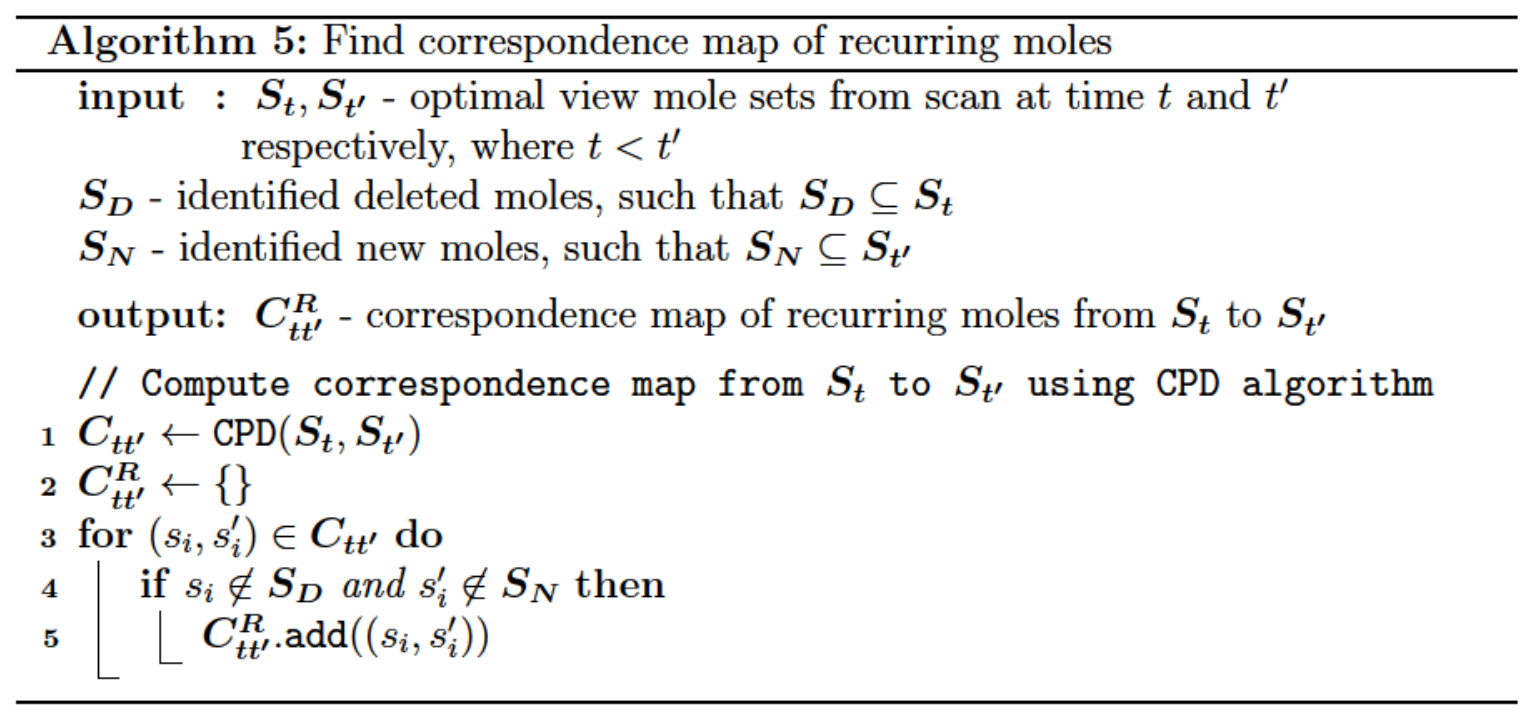

\subsection{Limitations of Temporal Scan Operations}

During the temporal scan operations we match moles across two $3 D$ mole maps that were created from two scans taken with time interval. During this time period the leg might have changed in size, the leg alignment on the platform was changed and possibly new moles appeared and some disappeared. All these changes to the leg and mole map combined make the temporal scan registration process complicated. The two main reasons for incorrect match is the error carry over from mole set creation (Section 4.3.2) and accuracy of CPD algorithm match (Section 4.3.2). Overcoming these limitations is out of this research scope. These errors of temporal scan operations fall into the following categories:

1. Recurring mole incorrectly matched to another recurring mole.

2. Recurring mole from reference set (from the first scan) was not matched to any mole and identified as disappeared. 
3. Recurring mole from matching set (from the second scan) was not matched to any mole and identified as new mole.

4. Disappeared mole was matched, i.e., was identified as recurring mole.

5. Newly appeared mole was matched, i.e., was identified as recurring mole.

\subsubsection{Error Carry Over from Mole Sets Creation}

One reason for failure in registering moles correctly is the error carry over from the Mole Sets creation described in Section 4.1.3.1.

During mole set creation step (Section 4.1.3), two mole sets were created for one mole, which was interpreted as two very close moles in the $3 D$ Mole Map (from Section 4.1.4, where one is a real one and one is phantom. The result is one of the following errors in correspondence map:

- If the mole did not appear in temporal exploration: the result is two moles were identified as deleted instead of one.

- If the mole was identified twice in the first scan and once in the second scan: the result is one mole matched, and the other phantom mole identified as deleted.

- If the mole was identified twice in the first scan and twice in the temporal scan: the result is two recurring mole matches were identified instead of one.

- If the mole did not appear in the initial scan, the result is two moles would identify as new instead of one. 
- If the mole has one representation in the first scan and two in the second scan, the result is one mole matched, and the other phantom mole identified as new.

\subsubsection{Error in Generation of Correspondence Map}

The CPD algorithm is probabilistic method, as such it is prone for errors, although it is a state of the art in registering non-rigid transformations in the presence of noise, outliers and missing points.

The combinations of the different scenarios, some of which are listed bellow, increase the errors in correspondence map from the CPD algorithm:

1. When a new mole appears very close to the place where a mole had disappeared

2. Large number of new moles compared with number of recurring moles

3. Large number of deleted moles compared with number of recurring moles

4. Moles were too close to each other, but in the resulting $3 D$ mole map (4.1.4) they appeared more close/farther apart

5. Number of recurring moles is too small to establish correct correspondence map

\subsection{Conclusions}

This chapter described an approach to automatically detect changes in multiple PSLs based on data acquired from the leg scanner. The approach incorporates LRF readings with camera images from known locations around the leg. The approach is divided 
into two main pipelines. The fist one, intra-exploration, where input data from one scan is processed, and a $3 D$ mole map model of the leg is created. The second one, temporal scan operations, where two mole maps from two scans are matched in order to assess changes of each individual mole.

Building the $3 D$ mole map of the leg consists of the following stages: (1) mole location in terms of surrounding LRF points in a single image, (2) mole matching across pairs of images (horizontal and vertical image pairs), (3) creation of mole sets i.e., finding same mole representation across all images, (4) selection of optimal image representation and recreation of a $3 D$ mole map. This pipeline heavily relies on the pinhole camera model, epipolar geometry, and Voronoi diagrams.

We assume that the majority of moles are present in two temporal scans, and that the leg did undergo some small changes. Two $3 D$ mole maps from consecutive scans are registered by CPD algorithm. Then, from the resulted correspondence map we extract deleted moles list, new moles list and recurring moles correspondence map. 


\section{Chapter 5}

\section{Simulation Design, Results and Discussion}

This is the initial stage of the research where a single LRF and one camera are used for leg scanning. The first step is to demonstrate that it is an overall good direction of research, and that this technique is applicable for screening of malignant nevi on legs. Developing a full-scale system is expensive and time consuming. Since this research is at its very early stage, we decided to create simulated hardware readings, i.e., camera and LRF readings. The software was built to analyze these readings as a proof of concept.

In this chapter we overview how we created simulated data of a leg with moles and hardware readings (Section 5.1). In Section 5.2 we describe how test cases were generated. In Section 5.4, we provide the results of our algorithm described in Chapter 4. The chapter is finalized with discussions of the results (Section 5.5). 


\subsection{Simulation Data Generator}

We developed a Simulation Data Generator (SDG) to create sets of artificial readings based on models of the leg with moles and our proposed hardware system. The SDG was designed with a focus on speed and simplicity in generation of many unique data sets. The multitude of unique data sets facilitates the testing of a wide range of scenarios, to better check the limits of our analytic algorithm (Chapter 4). Additionally, it allows the flexibility to create study cases as necessary.

Due to the stage of the research, we used a model that simplifies real patients' scenarios. The model of a patient's leg was approximated by the shape of a conical frustum. The moles were evenly distributed over the leg model and were represented solely based on their location (centre point). The reduction of complexity of the model introduces some limitations with regards to the moles' analysis (for malignancy) and comparison (for accurate registration of moles). Real images of moles have more information such as shape of the mole, as well as its colour and texture. The additional information could assist in mole comparison and matching, but due to the limitation of our model, we can only use mole locations for identification and registration.

\subsubsection{Leg's Shape}

If we look at a leg's shape, we can see that it resembles several conical frustums with different opening angles, attached at their bases Figure 5.1. Since the human leg has an opaque rigid shape with smooth changes and has no occluding contours [6], it is a reasonable approximation. For the purpose of our research, and without loss of 
generality, we simulated only one conical frustum.

During the time between consecutive scans, a patient's leg may undergo some transformations due to muscle growth, water retention, fat accumulation, or other factors. Note that a leg's changes are never expected to be vertical, since the target patients are usually mature adults who will not grow in height. Rather, the changes in

the legs' volumes are expected to occur through transformations along the horizontal plane. Our simulation can accommodate these changes of added or reduced volume of the conical frustum model by modifying the opening angle of the cone. The simulation is designed based on clinical information available about malignant melanoma [38].

The conical frustum is created from a cone with radius $R(100 \mathrm{~mm})$ and height $H(150 \mathrm{~mm})$. We use only part of this cone up to height $h(100 \mathrm{~mm})$, which is the conical frustum shape. In order to simulate a leg's change, during the second scan, the leg's radius changes by percentage of the original value.

\subsubsection{Moles}

We select an initial number of moles and distribute them randomly on the simulated leg with minimal distance between each two moles. Each mole is represented by a central point. This number was chosen since a mole with a radius of $2 \mathrm{~mm}$ or less does not have any clinical significance, and since we only know mole centre location.

When a random placement for a mole is generated, the algorithm first compares this location's distance to all previously generated moles. If it is farther that $5 \mathrm{~mm}$ from all other moles, the we set the mole at this location, otherwise, the algorithm selects new random place. This is repeated up to ten times. If all unsuccessful, the 


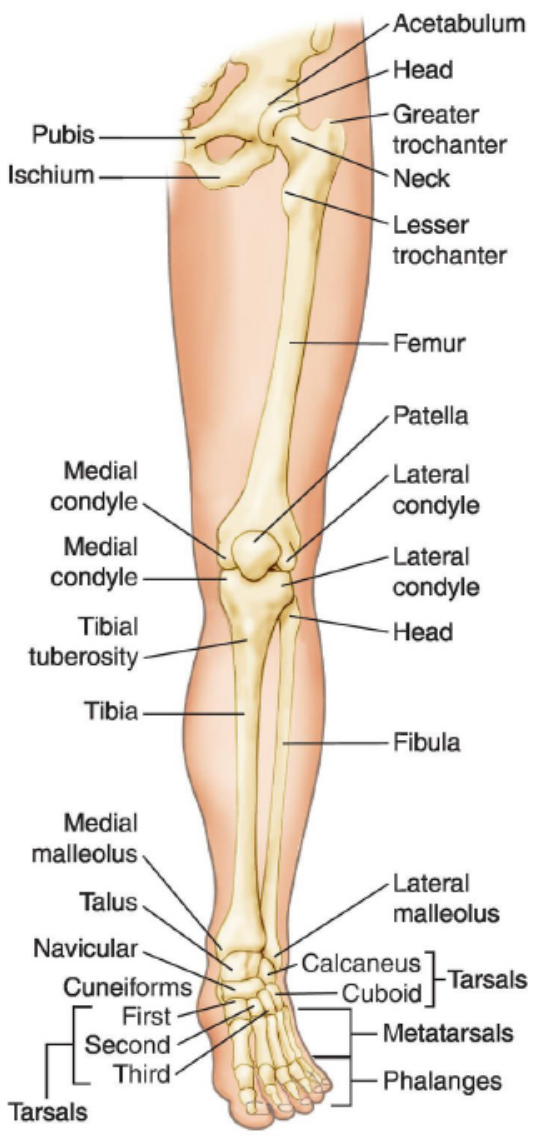

(a)

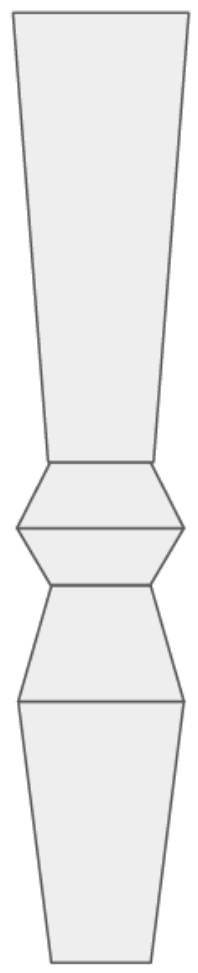

(b)

Figure 5.1: (a) Schematic representation of human leg [11]. (b) How to construct a shape similar to human leg with conical frustums with different opening angles attached at their bases. 
last random place becomes the mole's location.

For the simulation of the temporal scan the number of moles can change due to newly appearing and disappearing moles (for example, due to surgical excision). The number of deleted moles is selected randomly, up to 20 percent of the initial number of moles. Then we randomly select which moles among the pre-existing moles to delete. A random number of newly appearing moles is selected up to 20 percent of the initial number of moles.

\subsubsection{LRF data collection}

Our system resembles a turn-table for each layer [19], since the circular motion of the $\mathrm{LRF}$ and camera is mathematically equivalent to the rotation of a leg on a turn-table while camera and LRF are standing still. The rotation axis is exactly at the centre of the conical frustum. Thus, all horizontal LRF readings are identical and are equal to the distance from the LRF to the centre, minus radius of conical frustum at the local height Equation 3.1. We add a Gaussian noise to the LRF readings with mean 0 and standard deviation of $1 \mathrm{~mm}$. As well, we add a random error up to $1^{\circ}$ to the rotation angle $\theta_{i}^{L R F}$ and random error up to $1 \mathrm{~mm}$ to LRF height position $H_{j}$.

The platform's radius, for the purpose of our simulation, denoted by $\rho$, is 500 $\mathrm{mm}$ (i.e., the distance to the LRF and the camera's length from the center of the platform). 


\subsubsection{Camera Data Collection}

The camera is simulated as an ideal pinhole, with focal length denoted by $F$, and principal point denoted by $P$. The simulated camera rotates around the simulated leg and takes pictures at constant angle intervals, denoted by $\Delta L$, and layer intervals, denoted by $\Delta H$. Images taken by this camera consist of mole locations and LRF readings on the part of cone visible to the camera. For each camera stop, a simulated error was introduced into the camera's location, similar to LRF location error described in Section 5.1 .3 .

The simulated camera's intrinsic parameters were set to:

$$
\boldsymbol{K}=\left[\begin{array}{ccc}
300 & 0 & -60 \\
0 & 300 & 0 \\
0 & 0 & 1
\end{array}\right]
$$

With image size of $1200 \times 800$ pixels and image centre point at $(600,400)$.

\subsubsection{Camera's Transformation and Orientation}

The camera's extrinsic parameters (i.e., the exact orientation and translation of the camera with reference to world coordinates) are computed from the system's structure. The distance from the camera to platform centre is $\rho$, and its optical axis is pointing toward the centre of the platform in parallel to it. Consequently, the camera's location with respect to world coordinates is denoted by angle, $\theta_{i}^{C}$, with the world $x$-axis, platform radius $\rho$, and vertical height, $H j$. The camera's orientation 
$\mathcal{O}_{i}$ and location $\mathcal{L}_{i j}$, Equations 5.5, are derived from the constant initial rotation matrix $\boldsymbol{R}_{\boldsymbol{I}}$, Equation 5.2 , rotation matrix $\boldsymbol{R}_{Y}^{i}$ around $y$-axis in camera's coordinate system, Equations 5.3 , and translation vector $\boldsymbol{t}_{j}$, Equation 5.4 .

$$
\begin{aligned}
& \boldsymbol{R}_{\boldsymbol{I}}=\left[\begin{array}{ccc}
\cos (\pi / 2) & 0 & -\sin (\pi / 2) \\
0 & 1 & 0 \\
\sin (\pi / 2) & 0 & \cos (\pi / 2)
\end{array}\right] \\
& \boldsymbol{R}_{Y}^{i}=\left[\begin{array}{ccc}
\cos \left(\theta_{i}^{C}\right) & 0 & -\sin \left(\theta_{i}^{C}\right) \\
0 & 1 & 0 \\
\sin \left(\theta_{i}^{C}\right) & 0 & \cos \left(\theta_{i}^{C}\right)
\end{array}\right] \\
& \boldsymbol{t}_{j}=\left[\begin{array}{lll}
\rho & H_{j} & 0
\end{array}\right] \\
& \mathcal{L}_{i j}=-\boldsymbol{t}_{j}\left(\boldsymbol{R}_{Y}^{i}\right)^{T} \\
& \mathcal{O}_{i}=\left(\boldsymbol{R}_{\boldsymbol{Y}}^{i} \boldsymbol{R}_{\boldsymbol{I}}\right)^{T}
\end{aligned}
$$




\subsubsection{Simulation of Temporal Scan}

During Temporal Scan (i.e., a consecutive full scan of the leg) the system and the leg undergo changes since time has passed, patient's body has changed, and the leg position on the platform cannot be reproduced precisely to match that of the initial scan. In the simulation of time interval scan, we take into account changes to the leg and to the moles (disappeared moles and newly appeared ones). However, the simulated change in the position of leg on the scanning platform was not part of this research scope.

The number of disappeared or newly appeared moles in the temporal scan is a randomly generated percentage of the initial number of moles. The percentage of disappeared or newly appeared PSLs ranges between 0 and 20 .

\subsection{Scenarios Generator}

The simulation generates different scenarios to evaluate the performance of our algorithm from Chapter 4. The different scenarios are then used to test the limits of the algorithm's ability to register moles correctly within temporal scans. In addition, the scenarios are used to choose the scanning intervals for optimal analysis in terms of scanning time versus algorithm performance.

The scenarios were configured based on varying combinations of three parameters: initial number of moles, percent of leg's radius change in temporal scan, scanner step size. All the parameters are explained below: 
- Initial number of moles

- Values were chosen based on expected number of moles from the theory [38].

- Data Type: initial number of moles per data set. Values: 15, 20, 25, 30.

- Percent of leg's radius change in temporal scan, denoted by $\Delta R$

- The leg's shape is expected to change to an unpredictable extent in the time interval between two consecutive scans. We want to measure how the algorithm's performance is affected by the leg's radius change.

- Data Type: percent of leg's radius change in the temporal scan from the initial size. Values: $0 \%, 10 \%, 15 \%, 20 \%, 25 \%, 30 \%, 50 \%$.

- Scanner step size of data collection in terms of angle gaps between two LRF measurements or camera images, denoted by pairs of $(\Delta L, \Delta C)$.

- A measure of how often the imagining and distance measurement subsystems stop and take a picture/measurement of the leg. A smaller step size of LRF increases the accuracy of the $3 D$ modeled leg, as well affects the accuracy of matching mole pairs across two consecutive images (Section 4.1.2). A smaller step size of imaging sub-system also creates a larger number of images for analysis and each mole is captured from more angles, which beneficial for accurate analysis. On the other hand, a smaller step size increases the duration of the scan and the duration of the data processing. We want to find the largest step size, while keeping the accuracy unaffected. In addition, in our simulation, collecting images at a step size 
smaller than $10^{\circ}$ resulted in data processing time of more than 20 minutes per scenario (running on personal computer with processor speed $2.59 \mathrm{GHz}$ and 6 GB of RAM). Therefore, we decided not to go below this step size for imaging sub-system.

- Data Type: Horizontal step interval pairs for measurement and imaging sub systems, denoted by pair $(\Delta L, \Delta C)$ in degrees. Values: $\left(3^{\circ}, 10^{\circ}\right)$, $\left(5^{\circ}, 10^{\circ}\right),\left(7^{\circ}, 10^{\circ}\right),\left(10^{\circ}, 10^{\circ}\right),\left(12^{\circ}, 12^{\circ}\right),\left(14^{\circ}, 14^{\circ}\right),\left(16^{\circ}, 16^{\circ}\right)$.

For each value of the "initial number of moles" parameter, we created 20 scenarios. The scenarios were created from combinations of these three parameters.

\subsection{Additional Software Constraint}

When using real skin imaging for mole matching in adjacent image pairs, software should utilize image feature extraction algorithms to additionally validate mole pairs match (Section 4.1.2). In our simulated design each mole is described only by its center point. In order to compensate that, we decided to add an additional constraint on the mole sets creation (Section 4.1.3): mole sets that do not have at least three horizontal consecutive mole views are rejected. In other words, we require the scanner's step size for capturing images to be small enough such that in each two horizontally adjacent images have at least $66.67 \%$ overlap.

A short explanation of the field of view angle computation is given in Appendix A.2 when $50 \%$ overlap is required. 


\subsection{Results}

In this section we analyze the performance results of our software. The goal of our algorithm is to analyze two consecutive scans of the leg that were taken with time interval by our scanner (described in Chapter 3) and match between recurring moles, as well identify deleted and new moles. By correctly matching recurring moles, they can be compared for changes and evolution which is the most reliable indication of malignant melanoma (Section 2.3.1). Newly developed moles also possess risks of melanoma malignancy.

In order to evaluate the algorithm's accuracy, we count how many PSLs were correctly registered out of all PSLs' occurrences. The mole occurrences are divided into the following groups:

1. Number of recurring moles (i.e., moles that appear in both scans) denoted by $M^{R}$

2. Number of deleted moles (i.e., that appear only in the first scan) denoted by $M^{D}$

3. Number of new moles (i.e., was appear only in the second scan) denoted by $M^{N}$

We count correct registrations as follows:

1. Number of recurring moles that were correctly matched to itself in both scans, denoted by $M_{c}^{R}$

2. Number of deleted moles that were correctly identified as deleted, denoted by $M_{c}^{D}$ 
3. Number of new moles that were correctly identified as new, denoted by $M_{c}^{N}$

Therefore, the percent accuracy of our scan registration algorithm is defined by Equation 5.6. In order to calculate the algorithm performance, we calculate the average accuracy and standard deviation over 50 test cases. We present the results in graphs comparing the algorithm average accuracy with respect to different number of initial moles, scanner step size and leg's radius changes between the temporal scans.

$$
\text { Percent Accuracy }=\frac{\left(M_{c}^{R}+M_{c}^{D}+M_{c}^{N}\right) \cdot 100}{R_{c}+M_{c}+M_{c}}
$$

We calculated the average (mean) accuracy and the standard deviation over scenarios with identical parameters. The standard deviation denoted by $\sigma$ provides means of assessing the dispersion of the result accuracy. Given data set $x_{i} \in S$ and the size of the data set denoted by $N$. The mean is calculated by Equation 5.7 and the standard deviation by Equation 5.8 .

$$
\begin{gathered}
\bar{x}=\frac{\sum_{i=1}^{N} x_{i}}{N} \\
\sigma=\sqrt{\frac{\sum_{i=1}^{N}\left(x_{i}-\bar{x}\right)^{2}}{N-1}}
\end{gathered}
$$

We present only the most significant result findings in a graphical form. In each graph we arranged the average accuracy (\%) by initial number of moles, in order to demonstrate how the accuracy is affected by this parameter. In Figures 5.2 and 5.3 we keep the leg's radius change, denoted by $\Delta R$, as a constant set to $10 \%$ and $30 \%$ 


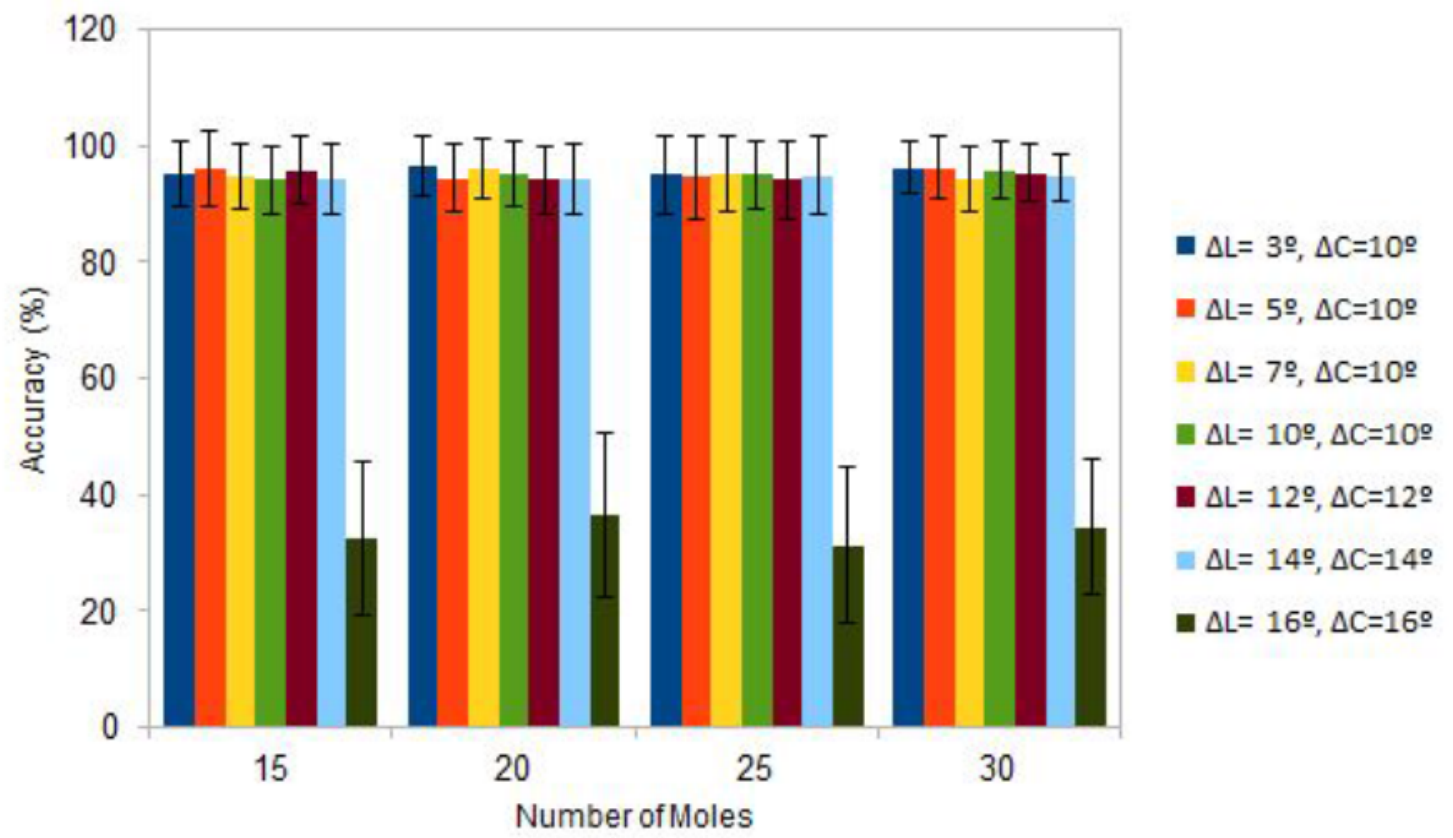

Figure 5.2: Average accuracy with radius difference $\Delta R=10 \%$. The results are divided into groups by initial number of moles on the leg model. The scanner step size denoted by pairs of LRF and Camera step sizes $(\Delta L, \Delta C)$ is varying.

respectively. The scanner step size, denoted by $(\Delta L, \Delta C)$, is varying.

The results presented in the Figures 5.4, 5.5, 5.6, 5.7, 5.8, 5.9 represent average accuracy for a specific scanner step size, denoted by $(\Delta L, \Delta C)$ in degrees, and the values are $\left(3^{\circ}, 10^{\circ}\right),\left(5^{\circ}, 10^{\circ}\right),\left(7^{\circ}, 10^{\circ}\right),\left(10^{\circ}, 10^{\circ}\right),\left(12^{\circ}, 12^{\circ}\right),\left(14^{\circ}, 14^{\circ}\right)$ respectively. In these graphs, we compare how the accuracy is affected by leg's radius change, denoted by $\Delta R$. 


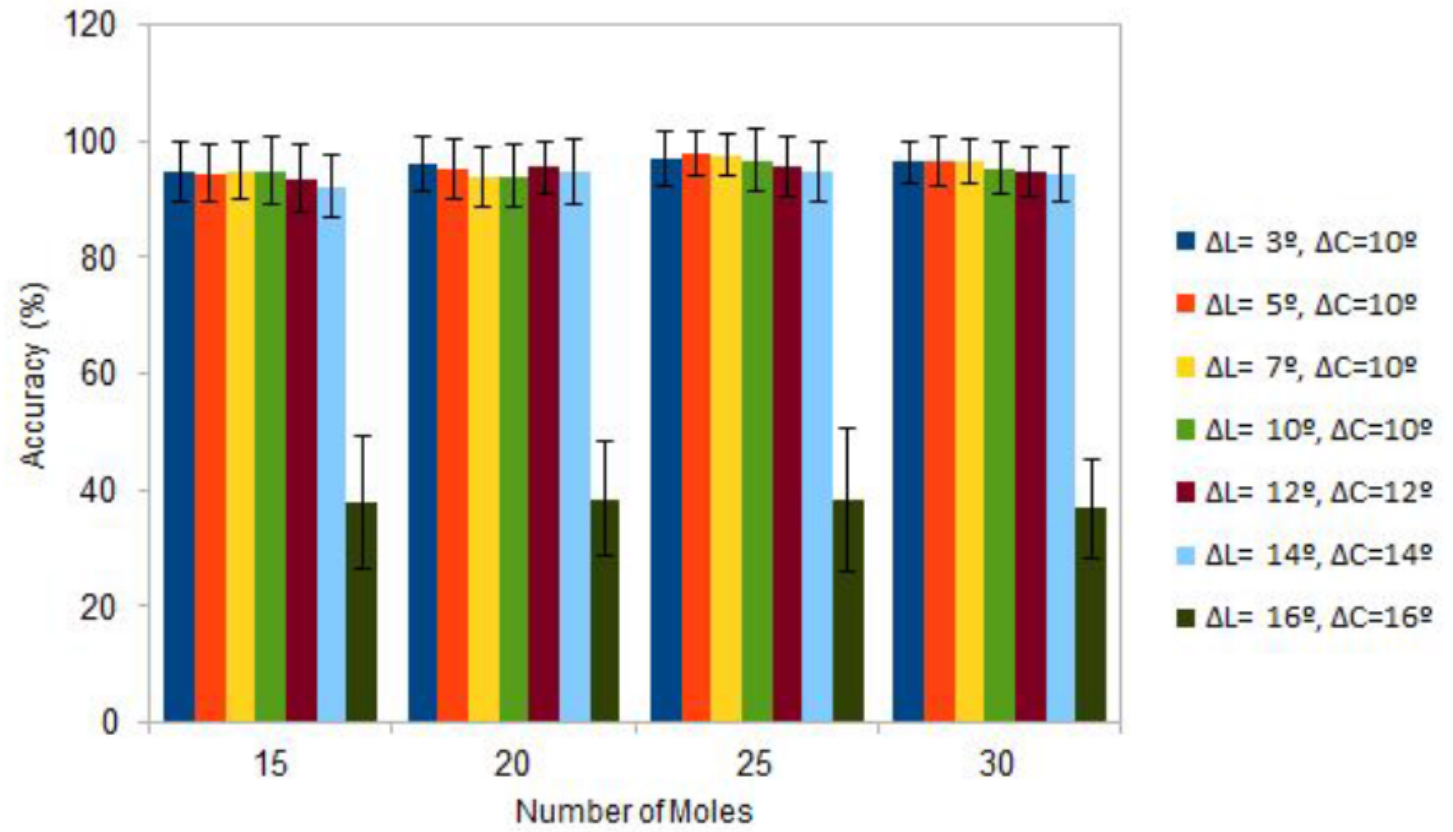

Figure 5.3: Average accuracy with radius difference $\Delta R=30 \%$. The results are divided into groups by initial number of moles on the leg model. The scanner step size denoted by pairs of LRF and Camera step sizes $(\Delta L, \Delta C)$ is varying. 


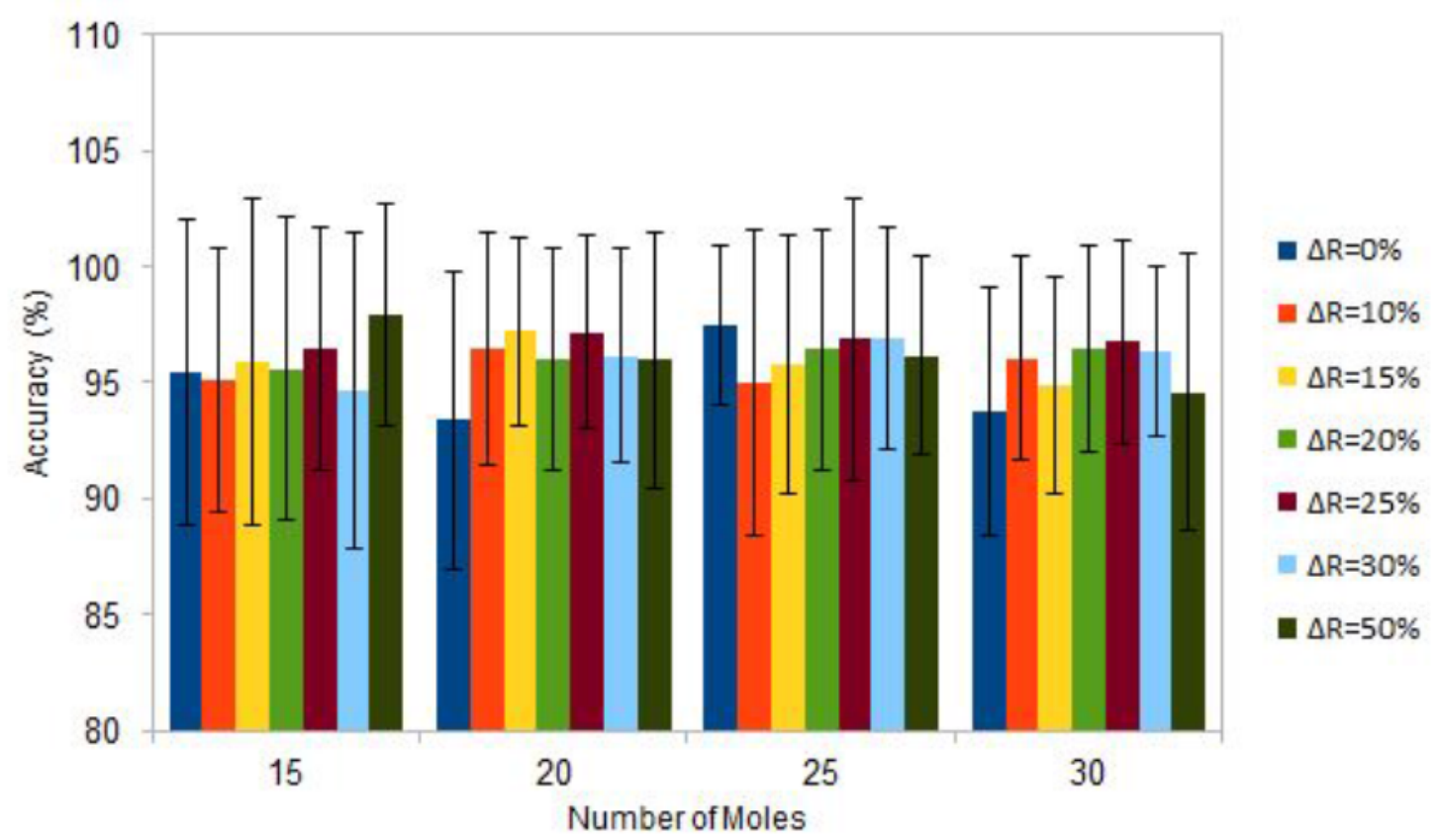

Figure 5.4: Average accuracy with scanning step size $(\Delta L, \Delta C)=\left(3^{\circ}, 10^{\circ}\right)$. The results are divided into groups by initial number of moles on the leg model. The percent of leg's radius growth in temporal scan is denoted by $\Delta R$. Overall average accuracy $95.95 \%$, minimal average accuracy per scenario type $93.40 \%$ and maximal average accuracy per scenario type $97.90 \%$. 


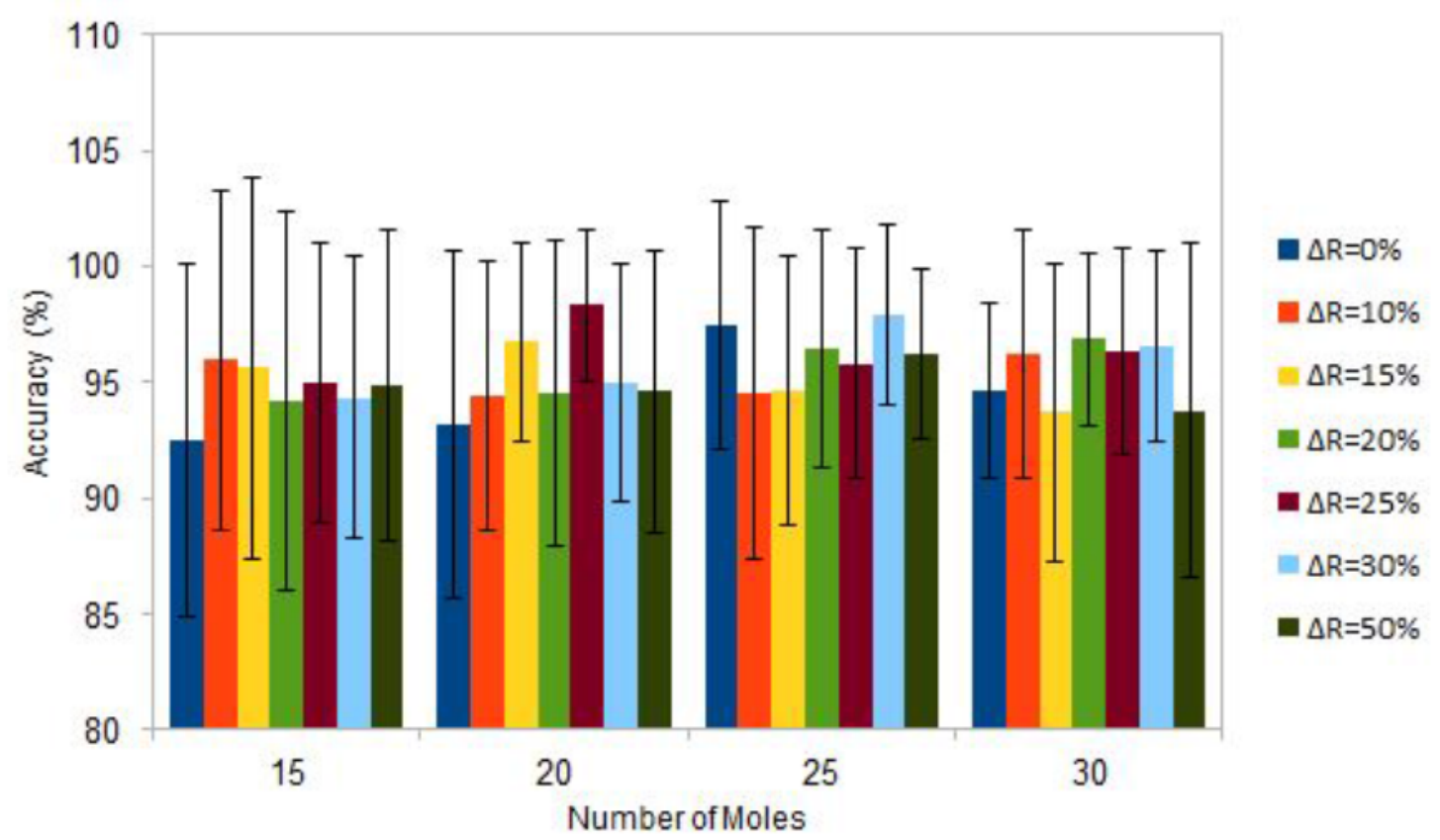

Figure 5.5: Average accuracy with scanning step size $(\Delta L, \Delta C)=\left(5^{\circ}, 10^{\circ}\right)$. The results are divided into groups by initial number of moles on the leg model. The percent of leg's radius growth in temporal scan is denoted by $\Delta R$. Overall average accuracy $95.36 \%$, minimal average accuracy per scenario type $92.47 \%$ and maximal average accuracy per scenario type $98.30 \%$. 


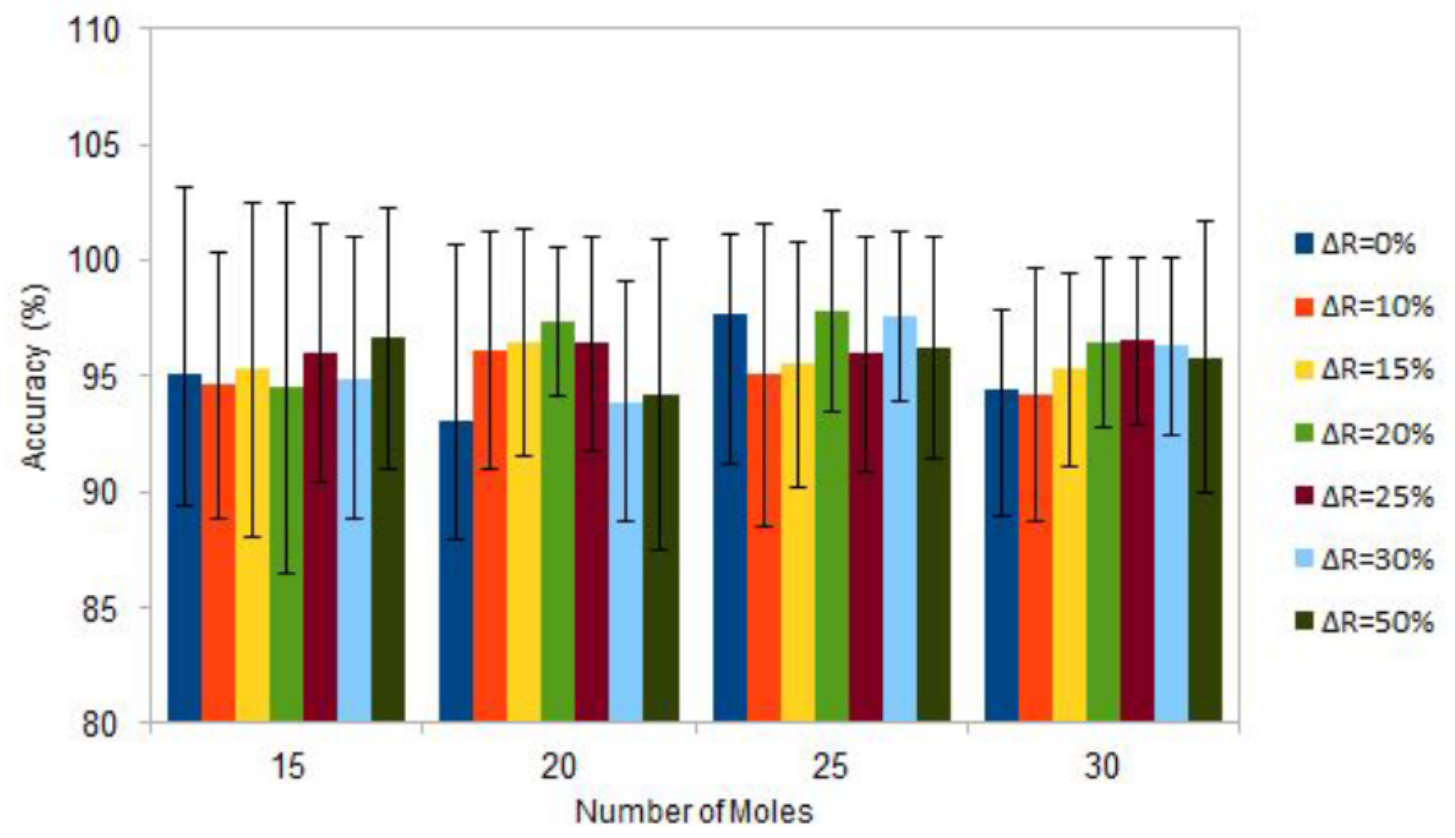

Figure 5.6: Average accuracy with scanning step size $(\Delta L, \Delta C)=\left(7^{\circ}, 10^{\circ}\right)$. The results are divided into groups by initial number of moles on the leg model. The percent of leg's radius growth in temporal scan is denoted by $\Delta R$. Overall average accuracy $95.68 \%$, minimal average accuracy per scenario type $93.10 \%$ and maximal average accuracy per scenario type $97.80 \%$. 


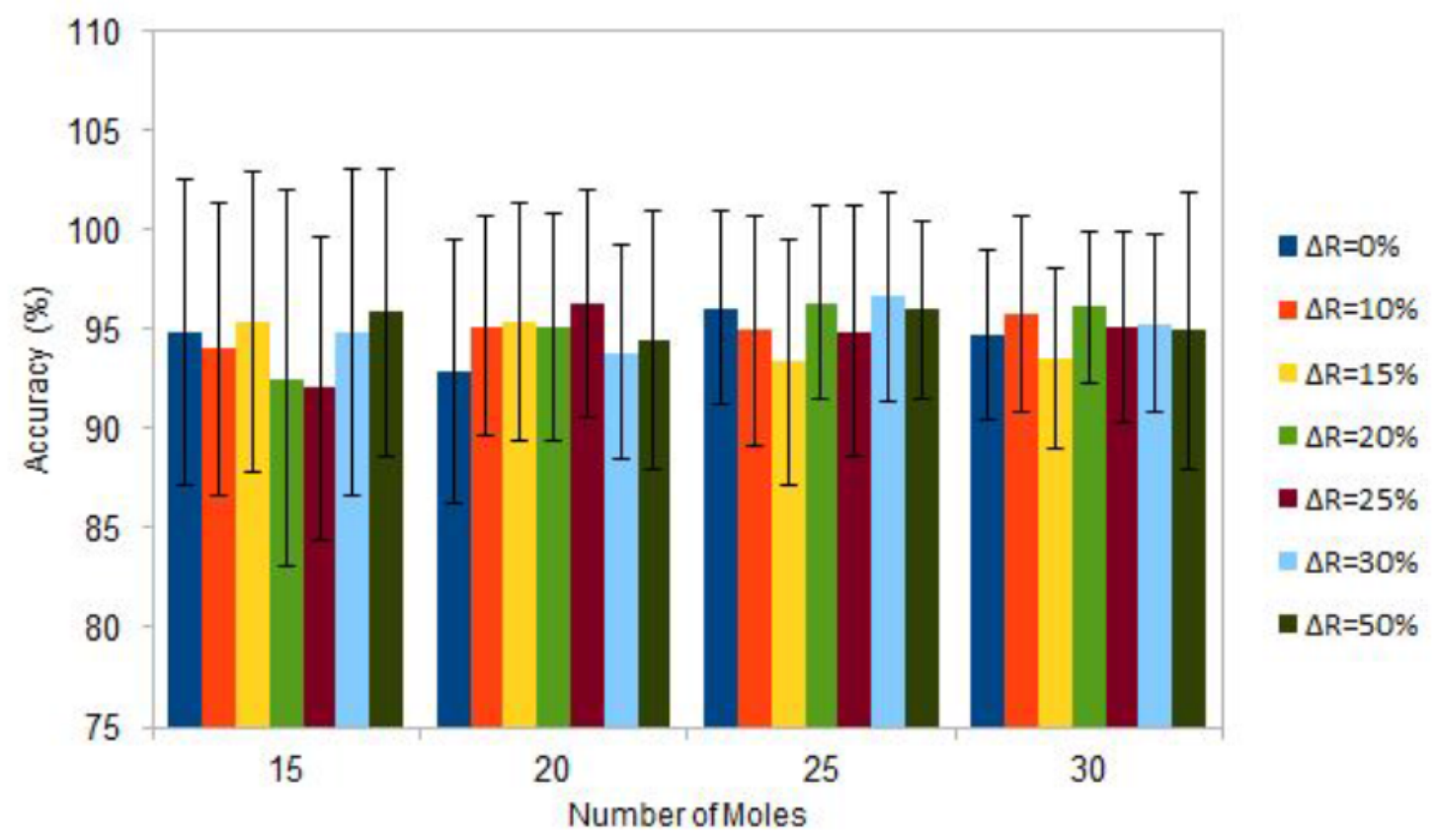

Figure 5.7: Average accuracy with scanning step size $(\Delta L, \Delta C)=\left(10^{\circ}, 10^{\circ}\right)$. The results are divided into groups by initial number of moles on the leg model. The percent of leg's radius growth in temporal scan is denoted by $\Delta R$. Overall average accuracy $94.87 \%$, minimal average accuracy per scenario type $92.05 \%$ and maximal average accuracy per scenario type $96.65 \%$. 


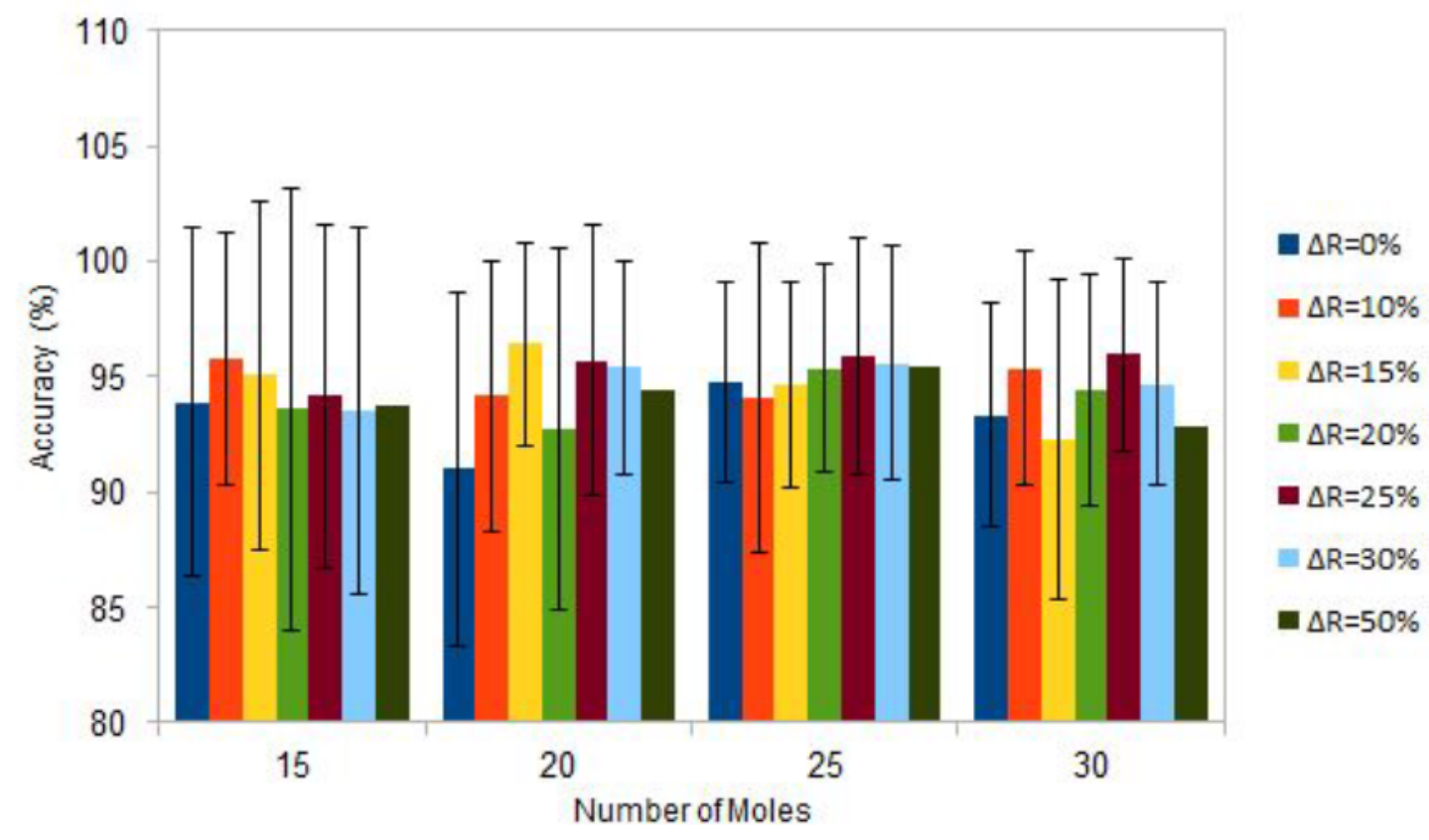

Figure 5.8: Average accuracy with scanning step size $(\Delta L, \Delta C)=\left(12^{\circ}, 12^{\circ}\right)$. The results are divided into groups by initial number of moles on the leg model. The percent of leg's radius growth in temporal scan is denoted by $\Delta R$. Overall average accuracy $94.43 \%$, minimal average accuracy per scenario type $91.00 \%$ and maximal average accuracy per scenario type $96.40 \%$. 


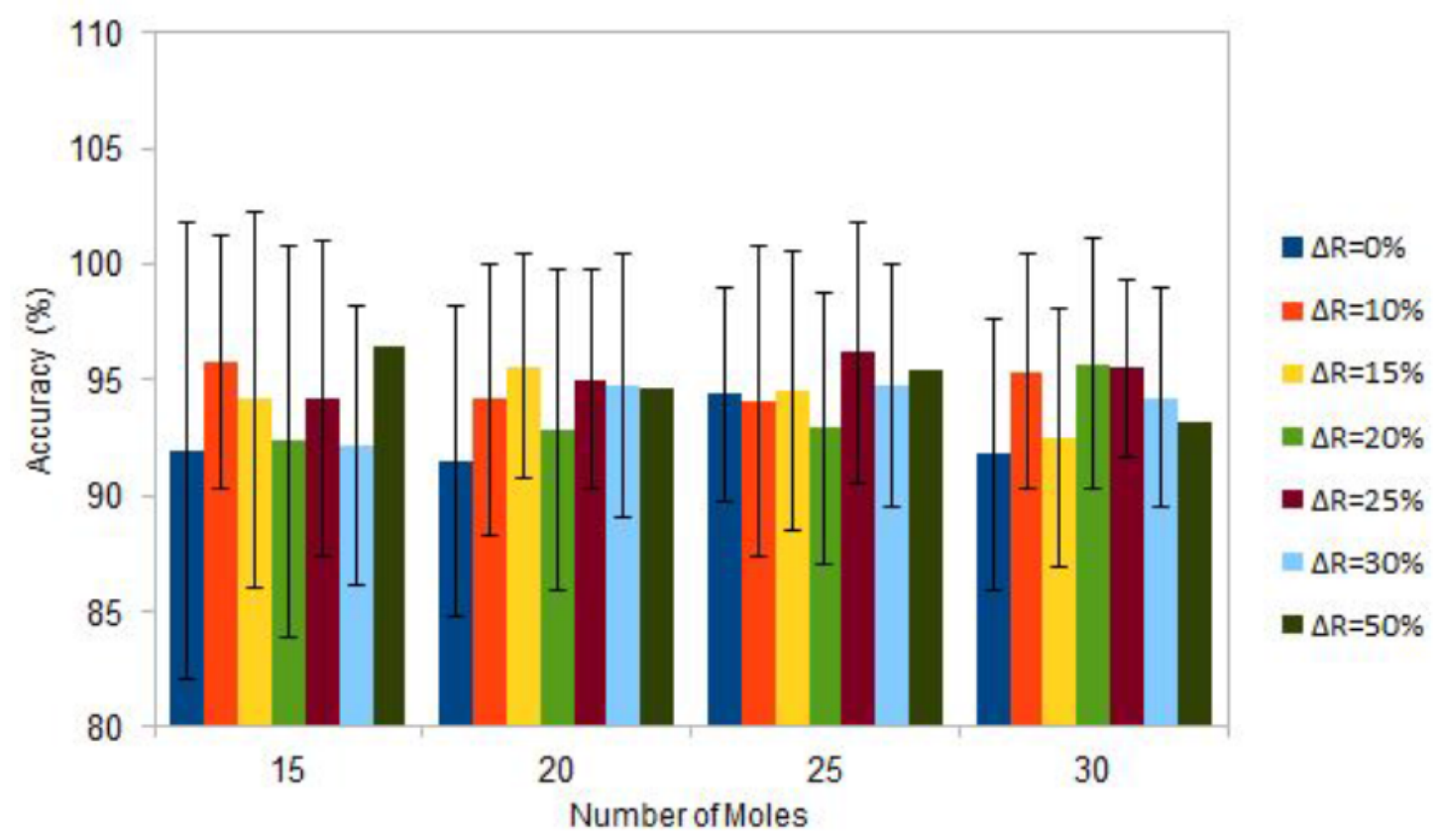

Figure 5.9: Average accuracy with scanning step size $(\Delta L, \Delta C)=\left(14^{\circ}, 14^{\circ}\right)$. The results are divided into groups by initial number of moles on the leg model. The percent of leg's radius growth in temporal scan is denoted by $\Delta R$. Overall average accuracy $94.14 \%$, minimal average accuracy per scenario type $91.45 \%$ and maximal average accuracy per scenario type $96.40 \%$. 


\subsection{Discussions}

From all the graphs in Section 5.4, we see a correlation between the scanner's accuracy and the initial number of moles. The scanner produces better accuracy with more recurring moles present. As can be seen, with 25-30 initial moles the accuracy is higher and with smaller standard deviation. This result correlates with CPD algorithm capabilities. The CPD algorithm generates more accurate correspondence maps between moles in two $3 D$ models when more recurring moles are present.

By analyzing Figures 5.2 and 5.3 we can see that scanner step size denoted by $(\Delta L$, $\Delta C)$ equal to $\left(3^{\circ}, 10^{\circ}\right),\left(5^{\circ}, 10^{\circ}\right),\left(7^{\circ}, 10^{\circ}\right),\left(10^{\circ}, 10^{\circ}\right),\left(12^{\circ}, 12^{\circ}\right),\left(14^{\circ}, 14^{\circ}\right)$, provide approximately consistent results values. In contrast, a scanner step size of $\left(16^{\circ}, 16^{\circ}\right)$ causes a significant drop in accuracy to below $40 \%$. The reason for this significant drop is that for higher step sizes above a threshold, the result images do not have $66.67 \%$ of horizontal overlap, and the moles do not appear on adjacent images.

From the graphs in Figures 5.4, 5.5, 5.6, 5.7, 5.8, and 5.9 for scanner step sizes $(\Delta L$, $\Delta C)$ equal to $\left(3^{\circ}, 10^{\circ}\right),\left(5^{\circ}, 10^{\circ}\right),\left(7^{\circ}, 10^{\circ}\right),\left(10^{\circ}, 10^{\circ}\right),\left(12^{\circ}, 12^{\circ}\right),\left(14^{\circ}, 14^{\circ}\right)$ respectively we can notice similarity in accuracy results with an overall accuracy average above 94\%. Our hypothesis, that with higher radius change between temporal scans the accuracy will reduce, was proven false. However, we can notice clearly that with a greater number of moles, the standard deviation is smaller, which can be attributed to the CPD performance, i.e., it registers two points' sets more accurately with a higher number of available matching points. The overall average accuracy is slightly smaller for scanner step size $(\Delta L, \Delta C)$ equal to $\left(12^{\circ}, 12^{\circ}\right)$ and $\left(14^{\circ}, 14^{\circ}\right)$, which matches, that more frequent scans should result in better accuracy, although the result is not 
significantly smaller, and more tests needs be performed to confirm this assumption firmly.

The accuracy correlation with scanner step size corresponds with our initial hypothesis, that a smaller scanner step size will result with higher accuracy, and there exists a threshold step size, after which the accuracy drops, since the adjacent images do not have enough overlap. From these results we conclude that, leg with radius up to $150 \mathrm{~mm} 11$, scanner step size of $(\Delta L, \Delta C)=\left(10^{\circ}, 10^{\circ}\right)$ provides the optimal accuracy - scanning (and processing) time trade-off. The average accuracy over all scenarios with this scanner step size was $94.87 \%$ with standard deviation of 6.10 . For a leg with a bigger radius, the step size must be adjusted to ensure at least a $50 \%$ (66.67\% with our additional constraint) overlap between images.

\footnotetext{
${ }^{1}$ In our test cases the initial leg size was $100 \mathrm{~mm}$ and in the temporal exploration after radius change of $\Delta R=50 \%$ the leg resulted with radius $150 \mathrm{~mm}$.
} 


\section{Chapter 6}

\section{Conclusion}

Melanoma is the most dangerous type of skin cancer. The main successful treatment method is excision of dysplastic nevus on early stages of melanoma. However, metastatic melanoma has no proven effective treatment methods to this day. Melanoma occurrence is not identical among men and women. For one, melanoma is more prevalent in men than women. Additionally, melanoma cancer location prevalence differs between men and women, where in men it mainly occurs on the torso, while in women it usually occurs on the lower limbs. Existing screening systems were designed to scan the torso.

In order to identify which moles are malignant melanoma, visual signs were identified to guide physicians and dermatologists. However, recognizing these signs and 
correctly categorizing moles as benign or malignant is largely dependent on the doctor's expertise. In addition, patients with high number of PSLs (over 50) have increased probability to develop melanoma skin cancer. Thus, it is a tedious and timeconsuming process to characterize all patients PSLs manually. The most reliable sign of melanoma development is mole evolution over period of time, primarily within three to six months. Whole body photography, and mole comparison to baseline images was suggested as a way to detect malignant moles. However, matching lesions to baseline images with the naked eye is, again, a time consuming and error prone task.

We performed a literature review and noticed that computer aided engineering solutions to detect evolution that would also benefit women (as women tend to have melanoma cases mostly on the legs) are missing, since legs are not properly scanned using whole body systems. We developed a leg scanner for mole registration and evolution detection while maintaining low-cost for equipment and operation.

\subsection{Contributions}

The following are the major contributions of this thesis:

- A system design that utilizes one camera and one LRF to scan a human leg. One of the objectives for this research was to create a low-cost scanner, that, (1) would be readily available for patients to have multiple scans within a year; and (2) could be used in remote locations where there is scarcity of expert doctors and health care budget. Developing a scanner from this perspective, 
we tried to reduce the complexity and the number of different parts. The result is a scanner with minimal equipment requirements. For instance, since highresolution cameras are costly, we showed how a LRF can be used to eliminate the need for multiple cameras.

- We invented an algorithm to be used along with a leg scanner, which solves the following problems:

- Creating a bidirectional correlation between the patient's PSL and the image, thus allowing doctors easily find the image based on lesion location on the leg, and, find the location of that mole on the leg, based on the image.

- PSL comparison over multiple scans that were performed with some time interval (and the leg could have been changed). The outcome allows doctors to detect new lesions, find lesions that disappeared and compare the same lesion over time.

These solutions help with the automatic detection of evolving lesions, as well as provide doctors a way to easily find how lesions looked in the past as a baseline image for accurate comparison.

\subsection{Limitations}

Our lower limbs scanner presented here has its limitations due to the nature of the algorithms and hardware design. Here are the main identified shortcomings: 
- Patient movement - A patient should be able to stand still on one leg for the scan duration while holding handrails. At this point, the algorithms were developed with the assumption of very little to no motion of patient's legs during the scan. The use of an additional LRF around the leg potentially could help identify patient's movements, and help correlate the images with respect to the movements. Development of that approach was beyond the scope of current research.

- Leg's dimensions - There is a limitation on patient's legs size due to the circumference of system's base and required minimal distance from camera to skin for sharp images.

\subsection{Future Work}

Among future work directions we highlight the following:

- Prototype - Building the prototype of presented system. The system design was simulated, and the algorithmic solution part was based on simulated hardware results. A real prototype has to be built and tested for the integrity of this research.

- Overcoming leg movement - In this research we assume no leg movements in our calculations. Enhancing the system to detect and measure leg's movements will allow more accurate PSLs registration. An example of such mechanism is to use six LRFs around the perimeter of the upper part of the leg. They 
will detect leg's movement along with the scanning results, thus allowing to the software adjust the PSLs registration.

- Creating mechanism of adjusting initial scanning point. Initial scanning point depends on where the centre of lateral malleolus bone was marked and how the leg was placed on the platform by the operator. The system should correct the two scans to start from the same location on the leg.

- Incorporate the software for automatic mole recognition on skin images and identify mole centres. Our algorithm assumes that all the preprocessing work on the images was already done, and PSLs were located. Based on our literature review, there exist different approaches for this image preprocessing. For full integrity of our work, the best suited pre-processing solution should be selected to precede the algorithm we suggested in Chapter 4 .

- Integrate a PSL analysis algorithm. Integrate an algorithm that receives as input optimal views of moles, extracts visible features, and utilizes artificial intelligence algorithms. This algorithm will serve two purposes: (i) create additional validation to the 3D mole maps registration results from temporal scan operations; (ii) analyze each mole for preliminary melanoma signs.

- Integrate PSLs evolution detection algorithm Once lesions are matched on consecutive scans, they should be analyzed for size change, as well as their colour change. These changes will provide incentives for lesion evolution and a measurement of its malignancy.

- Testing the system on patients. In order to complete the life-cycle of this product development it must be tested on real patients before it is released for 
mass production and use.

Although Canada is a developed country with great medicine, residents of rural areas have limited access to specialized medical professionals including dermatologists. We designed a low-cost lower limb scanner that could benefit about 1300 of women's a year across Canada [53] by helping detect new cases of melanoma on legs. The scanning method is not invasive, has no side effects to the patient, and requires minimal operator assistance. Therefore, it can be very beneficial for the Canadian population of women. Another potential use of this scanner design is tele-medicine, with dermatologist sitting hundreds of kilometers away from the patient, while navigating a camera around the patient's leg to take closer look at specific PSLs. 


\section{List of References}

[1] Matlab and statistics toolbox release 2016a, 2016.

[2] Naheed R Abbasi, Helen M Shaw, Darrell S Rigel, Robert J Friedman, William H McCarthy, Iman Osman, Alfred W Kopf, and David Polsky. Early diagnosis of cutaneous melanoma: revisiting the abcd criteria. Jama, 292(22):2771-2776, 2004.

[3] Giuseppe Argenziano, Gabriella Fabbrocini, Paolo Carli, Vincenzo De Giorgi, Elena Sammarco, and Mario Delfino. Epiluminescence microscopy for the diagnosis of doubtful melanocytic skin lesions: comparison of the abcd rule of dermatoscopy and a new 7-point checklist based on pattern analysis. Archives of dermatology, 134(12):1563-1570, 1998.

[4] Jeremy P Banky, John W Kelly, Dallas R English, Josephine M Yeatman, and John P Dowling. Incidence of new and changed nevi and melanomas detected using baseline images and dermoscopy in patients at high risk for melanoma. Archives of dermatology, 141(8):998-1006, 2005.

[5] François Blais. Review of 20 years of range sensor development. Journal of electronic imaging, 13(1):231-244, 2004. 
[6] Edmond Boyer. Object models from contour sequences. In European Conference on Computer Vision, pages 109-118. Springer, 1996.

[7] Kevin Q Brown. Voronoi diagrams from convex hulls. Information Processing Letters, 9(5):223-228, 1979.

[8] Taeg Sang Cho, William T Freeman, and Hensin Tsao. A reliable skin mole localization scheme. In Computer Vision, 200\%. ICCV 2007. IEEE 11th International Conference on, pages 1-8. IEEE, 2007.

[9] GR Day and RH Barbour. Automated skin lesion screening-a new approach. Melanoma research, 11(1):31-35, 2001.

[10] Atam P Dhawan. Apparatus and method for skin lesion examination, September 15 1992. US Patent 5,146,923.

[11] Taber's Medical Dictionary. Leg. https://www.tabers.com/tabersonline/ view/Tabers-Dictionary/765889/all/leg. Accessed December, 2018.

[12] Stephan Dreiseitl and Michael Binder. Do physicians value decision support? a look at the effect of decision support systems on physician opinion. Artificial intelligence in medicine, 33(1):25-30, 2005.

[13] Rhett J Drugge, Chi Nguyen, Elizabeth D Drugge, Luciana Gliga, Patrick A Broderick, Steve A McClain, and Christopher C Brown. Melanoma screening with serial whole body photographic change detection using melanoscan $₫$ technology. Dermatology online journal, 15(6), 2009. 
[14] Rhett J Drugge, Chi Nguyen, Luciana Gliga, and Elizabeth D Drugge. Clinical pathway for melanoma detection using comprehensive cutaneous analysis with melanoscan®. Dermatology online journal, 16(8), 2010.

[15] Gabriella Fabbrocini, Valerio De Vita, Sara Cacciapuoti, Giuseppe Di Leo, Consolatina Liguori, Alfredo Paolillo, Antonio Pietrosanto, and Paolo Sommella. Automatic diagnosis of melanoma based on the 7-point checklist. In Computer Vision Techniques for the Diagnosis of Skin Cancer, pages 71-107. Springer, 2014.

[16] Neal E Feit, Stephen W Dusza, and Ashfaq A Marghoob. Melanomas detected with the aid of total cutaneous photography. British Journal of Dermatology, 150(4):706-714, 2004.

[17] Daniel P Filiberti, Paolo Bellutta, Phillip Ngan, and Douglas A Perednia. Efficient segmentation of large-area skin images: an overview of image processing. Skin Research and Technology, 1(4):200-208, 1995.

[18] DP Filiberti, JA Gaines, P Bellutta, P Ngan, and DA Perednia. Efficient segmentation of large-area skin images: a statistical evaluation. Skin Research and Technology, 3(1):28-35, 1997.

[19] Andrew W Fitzgibbon, Geoff Cross, and Andrew Zisserman. Automatic 3d model construction for turn-table sequences. In European Workshop on 3D Structure from Multiple Images of Large-Scale Environments, pages 155-170. Springer, 1998. 
[20] Robert J Friedman, Darrell S Rigel, and Alfred W Kopf. Early detection of malignant melanoma: The role of physician examination and self-examination of the skin. CA: a cancer journal for clinicians, 35(3):130-151, 1985.

[21] J Frühauf, B Leinweber, R Fink-Puches, V Ahlgrimm-Siess, E Richtig, IH Wolf, A Niederkorn, F Quehenberger, and R Hofmann-Wellenhof. Patient acceptance and diagnostic utility of automated digital image analysis of pigmented skin lesions. Journal of the European Academy of Dermatology and Venereology, 26(3):368-372, 2012.

[22] W Harris Green, Steven Q Wang, and Armand B Cognetta. Total-body cutaneous examination, total-body photography, and dermoscopy in the care of a patient with xeroderma pigmentosum and multiple melanomas. Archives of dermatology, 145(8):910-915, 2009.

[23] Exposure guide. Camera basics. https://www.exposureguide.com. Accessed February, 2018.

[24] Allan C Halpern. Total body skin imaging as an aid to melanoma detection. In Seminars in cutaneous medicine and surgery, volume 22 , pages $2-8$. No longer published by Elsevier, 2003.

[25] Allan C Halpern, Ashfaq A Marghoob, Thomas W Bialoglow, William Witmer, and William Slue. Standardized positioning of patients (poses) for whole body cutaneous photography. Journal of the American Academy of Dermatology, 49(4):593-598, 2003. 
LIST OF REFERENCES

[26] Richard Hartley and Andrew Zisserman. Multiple view geometry in computer vision. Cambridge university press, 2003.

[27] Heng Huang and Paul Bergstresser. A new hybrid technique for dermatological image registration. In Bioinformatics and Bioengineering, 2007. BIBE $200 \%$. Proceedings of the 7th IEEE International Conference on, pages 1163-1167. IEEE, 2007.

[28] Michael J Jones and James M Rehg. Statistical color models with application to skin detection. International Journal of Computer Vision, 46(1):81-96, 2002.

[29] John W Kelly, Josephine M Yeatman, Cheryl Regalia, Grahame Mason, and Amanda P Henham. A high incidence of melanoma found in patients with multiple dysplastic naevi by photographic surveillance. The Medical journal of Australia, 167(4):191-194, 1997.

[30] Harald Kittler, Pascale Guitera, Elisabeth Riedl, Michelle Avramidis, Ligia Teban, Manfred Fiebiger, Rickard A Weger, Markus Dawid, and Scott Menzies. Identification of clinically featureless incipient melanoma using sequential dermoscopy imaging. Archives of dermatology, 142(9):1113-1119, 2006.

[31] Konstantin Korotkov and Rafael Garcia. Computerized analysis of pigmented skin lesions: a review. Artificial intelligence in medicine, 56(2):69-90, 2012.

[32] Konstantin Korotkov, Josep Quintana, Susana Puig, Josep Malvehy, and Rafael Garcia. A new total body scanning system for automatic change detection in multiple pigmented skin lesions. IEEE transactions on medical imaging, 34(1):317$338,2015$. 
[33] P. D. Kovesi. MATLAB and Octave functions for computer vision and image processing. Available from: <http://www.peterkovesi.com/matlabfns/>.

[34] Tim K Lee, M Stella Atkins, Michael A King, Savio Lau, and David I McLean. Counting moles automatically from back images. IEEE Transactions on Biomedical Engineering, 52(11):1966-1969, 2005.

[35] RM MacKie and VR Doherty. Seven-point checklist for melanoma. Clinical and experimental dermatology, 16(2):151-152, 1991.

[36] R Marchesini, A Bono, C Bartoli, M Lualdi, S Tomatis, and N Cascinelli. Optical imaging and automated melanoma detection: questions and answers. Melanoma research, 12(3):279-286, 2002.

[37] Ashfaq A Marghoob, Lucinda D Swindle, Claudia ZM Moricz, Fitzgeraldo A Sanchez Negron, Bill Slue, Allan C Halpern, and Alfred W Kopf. Instruments and new technologies for the in vivo diagnosis of melanoma. Journal of the American Academy of Dermatology, 49(5):777-797, 2003.

[38] Svetomir N Markovic, Lori A Erickson, Ravi D Rao, Robert R McWilliams, Lisa A Kottschade, Edward T Creagan, Roger H Weenig, Jennifer L Hand, Mark R Pittelkow, Barbara A Pockaj, et al. Malignant melanoma in the 21st century, part 1: epidemiology, risk factors, screening, prevention, and diagnosis. In Mayo Clinic Proceedings, volume 82, pages 364-380. Elsevier, 2007.

[39] Svetomir N Markovic, Lori A Erickson, Ravi D Rao, Roger H Weenig, Barbara A Pockaj, Aditya Bardia, Celine M Vachon, Steven E Schild, Robert R McWilliams, Jennifer L Hand, et al. Malignant melanoma in the 21st century, part 2: staging, 
prognosis, and treatment. In Mayo Clinic Proceedings, volume 82, pages 490-513. Elsevier, 2007.

[40] JA McGrath, RAJ Eady, and FM Pope. Anatomy and organization of human skin. Rooks textbook of dermatology, 3:1-15, 2004.

[41] Bruce Mcgregor. Automatic registration of images of pigmented skin lesions. Pattern Recognition, 31(6):805-817, 1998.

[42] Hengameh Mirzaalian, Ghassan Hamarneh, and Tim K Lee. A graph-based approach to skin mole matching incorporating template-normalized coordinates. In Computer Vision and Pattern Recognition, 2009. CVPR 2009. IEEE Conference on, pages 2152-2159. IEEE, 2009.

[43] Hengameh Mirzaalian, Tim K Lee, and Ghassan Hamarneh. Uncertainty-based feature learning for skin lesion matching using a high order mrf optimization framework. In International Conference on Medical Image Computing and Computer-Assisted Intervention, pages 98-105. Springer, 2012.

[44] Hengameh Mirzaalian, Tim K Lee, and Ghassan Hamarneh. Spatial normalization of human back images for dermatological studies. IEEE journal of biomedical and health informatics, 18(4):1494-1501, 2014.

[45] Hengameh Mirzaalian, Tim K Lee, and Ghassan Hamarneh. Skin lesion matching using jacobian based descriptors for topology preservation. In Biomedical Imaging (ISBI), 2015 IEEE 12th International Symposium on, pages 1407-1410. IEEE, 2015. 
[46] Hengameh Mirzaalian, Tim K Lee, and Ghassan Hamarneh. Skin lesion tracking using structured graphical models. Medical image analysis, 27:84-92, 2016.

[47] Andriy Myronenko and Xubo Song. Point set registration: Coherent point drift. IEEE transactions on pattern analysis and machine intelligence, 32(12):2262$2275,2010$.

[48] Andriy Myronenko, Xubo Song, and Miguel A Carreira-Perpinán. Non-rigid point set registration: Coherent point drift. In Advances in Neural Information Processing Systems, pages 1009-1016, 2007.

[49] Song X. Myronenko A. Coherent point drift (cpd) project page. https://sites . google.com/site/myronenko/research/cpd. Accessed October, 2018.

[50] KS Nehal, SA Oliveria, AA Marghoob, PJ Christos, S Dusza, JS Tromberg, and AC Halpern. Use of and beliefs about baseline photography in the management of patients with pigmented lesions: a survey of dermatology residency programmes in the united states. Melanoma Research, 12(2):161-167, 2002.

[51] Marija J Norusis. SPSSIX Advanced statistics guide. McGraw-Hill, 1985.

[52] Canadian Cancer Societys Advisory Committee on Cancer Statistics. Canadian cancer statistics 2017. http://www.cancer.ca/ Canadian-CancerStatistics-2017-EN.pdf, June 2017. Accessed March, 2018 .

[53] Canadian Cancer Societys Advisory Committee on Cancer Statistics. Canadian cancer statistics 2018. http://www.cancer.ca/ /media/cancer.ca/CW/ cancer $\% 20$ information/cancer $\% 20101 /$ Canadian $\% 20$ cancer $\% 20$ statistics/ 
Canadian-Cancer-Statistics-2018-EN.pdf, June 2018. Accessed October, 2018.

[54] Douglas A Perednia and Raymond G White. Automatic registration of multiple skin lesions by use of point pattern matching. Computerized medical imaging and graphics, 16(3):205-216, 1992.

[55] Douglas A Perednia, Raymond G White, and Robert A Schowengerdt. Localization of cutaneous lesions in digital images. Computers and biomedical research, 22(4):374-392, 1989.

[56] Simon JD Prince. Computer vision: models, learning, and inference. Cambridge University Press, 2012.

[57] Health professionals. The international skin imaging collaboration: Melanoma project. https://isic-archive.com/. Accessed January, 2018.

[58] Marius Rademaker and Amanda Oakley. Digital monitoring by whole body photography and sequential digital dermoscopy detects thinner melanomas. Journal of primary health care, 2(4):268-272, 2010.

[59] M Riech. Skin Cancer Detection System Registration of moles in skin images. PhD thesis, PhD thesis, Eindhoven University of Technology, Netherlands, 1997. $1,1997$.

[60] Edgar Rieger, H Peter Soyer, Claus Garbe, Petra Büttner, Regina Kofler, Jürgen Weiss, Ulrich Stocker, Sabine Krüger, Marianne Roser, Jörg Weckbecker, et al. Overall and site-specific risk of malignant melanoma associated with nevus counts at different body sites: A multicenter case-control study of the german central 
malignant-melanoma registry. International journal of cancer, 62(4):393-397, 1995.

[61] Darrell S Rigel, Julie Russak, and Robert Friedman. The evolution of melanoma diagnosis: 25 years beyond the abcds. CA: a cancer journal for clinicians, 60(5):301-316, 2010.

[62] Juha Roning and Marcel Riech. Registration of nevi in successive skin images for early detection of melanoma. In Pattern Recognition, 1998. Proceedings. Fourteenth International Conference on, volume 1, pages 352-357. IEEE, 1998.

[63] Bernard W Silverman. Density estimation for statistics and data analysis, volume 26. CRC press, 1986.

[64] William Slue, Alfred W Kopf, and Jason K Rivers. Total-body photographs of dysplastic nevi. Archives of dermatology, 124(8):1239-1243, 1988.

[65] American Cancer Society. Skin structure. https://www.cancer.org/cancer/ melanoma-skin-cancer/about/what-is-melanoma.html. Accessed March, 2018.

[66] Vitaly Terushkin, Susan A Oliveria, Ashfaq A Marghoob, and Allan C Halpern. Use of and beliefs about total body photography and dermatoscopy among us dermatology training programs: an update. Journal of the American Academy of Dermatology, 62(5):794-803, 2010.

[67] L Thomas, P Tranchand, F Berard, T Secchi, C Colin, and G Moulin. Semiological value of abcde criteria in the diagnosis of cutaneous pigmented tumors. Dermatology, 197(1):11-17, 1998. 
[68] Hensin Tsao, Jeannette M Olazagasti, Kelly M Cordoro, Jerry D Brewer, Susan C Taylor, Jeremy S Bordeaux, Mary-Margaret Chren, Arthur J Sober, Connie Tegeler, Reva Bhushan, et al. Early detection of melanoma: reviewing the abcdes. Journal of the American Academy of Dermatology, 72(4):717-723, 2015.

[69] Ezgi Unlu, Bengu N Akay, and Cengizhan Erdem. Comparison of dermatoscopic diagnostic algorithms based on calculation: The abcd rule of dermatoscopy, the seven-point checklist, the three-point checklist and the cash algorithm in dermatoscopic evaluation of melanocytic lesions. The Journal of dermatology, 41(7):598-603, 2014.

[70] Malene E Vestergaard and Scott W Menzies. Automated diagnostic instruments for cutaneous melanoma. In Seminars in cutaneous medicine and surgery, volume 27, pages 32-36. Frontline Medical Communications, 2008.

[71] ME Vestergaard, PHPM Macaskill, PE Holt, and SW Menzies. Dermoscopy compared with naked eye examination for the diagnosis of primary melanoma: a meta-analysis of studies performed in a clinical setting. British Journal of Dermatology, 159(3):669-676, 2008.

[72] Vladimir Vezhnevets, Vassili Sazonov, and Alla Andreeva. A survey on pixelbased skin color detection techniques. In Proc. Graphicon, volume 3, pages 85-92. Moscow, Russia, 2003.

[73] Holger Voigt and Richarda Claßen. Topodermatographic image analysis for melanoma screening and the quantitative assessment of tumor dimension parameters of the skin. Cancer, 75(4):981-988, 1995. 
[74] Raymond G White and Douglas A Perednia. Automatic derivation of initial match points for paired digital images of skin. Computerized medical imaging and graphics, 16(3):217-225, 1992.

[75] Raymond G White, Douglas A Perednia, and Robert A Schowengerdt. Automated feature detection in digital images of skin. Computer Methods and Programs in Biomedicine, 34(1):41-60, 1991.

[76] G Zouridakis, M Doshi, and N Mullani. Early diagnosis of skin cancer based on segmentation and measurement of vascularization and pigmentation in nevoscope images. In Engineering in Medicine and Biology Society, 2004. IEMBS'04. 26th Annual International Conference of the IEEE, volume 1, pages 1593-1596. IEEE, 2004. 
Appendices 


\section{Appendix A}

\section{Appendix}

\section{A.1 Coherent Point Drift Registration Algorithm}

Coherent Point Drift (CDP) algorithm by Myronenko et al. [48, 47] provides probabilistic solution for multidimensional point set registration for rigid and non rigid transformations. The algorithm accepts two points sets: first represents the Gaussian Mixture Models (GMM) centroids, denoted by $\mathcal{M}$, second represents a data point set, denoted by $\mathcal{S}$. The GMM centroids are forced to move coherently as a group (which preserves group topological structure) in order to fit the data points. The cost function is optimized by Expectation Maximization (EM) algorithm. The computational complexity of this algorithm can be reduced to linear. We will follow the following notations:

1. $M$ and $N$ are number of points in point sets $\mathcal{M}$ and $\mathcal{S}$ respectively.

2. $D$ number of point sets dimensions. 
3. $m_{i} \in \mathcal{M}, s_{j} \in \mathcal{S}$ represent arbitrary $D$ dimensional points from corresponding point sets.

4. $w \in[0,1]$ weight of the uniform distribution.

5. $\mathcal{T}(\mathcal{M}, \theta)$ Transformation $\mathcal{T}$ applied to point set $\mathcal{M}$, where $\theta$ represents set of the transformation parameters.

6. 1 and $\boldsymbol{I}$ are column vector of ones and identity matrix respectively.

7. $\theta=a, \boldsymbol{R}, \boldsymbol{t}$ The transformation parameters $\theta$ can be separated to a scale $a$, rotation matrix $\boldsymbol{R}$, and translation vector $\boldsymbol{t}$. While it initialized to $\theta_{0}=1, \boldsymbol{I}, \mathbf{0}$.

8. $\operatorname{svd}(\boldsymbol{A})$ stands for the singular value decomposition of $\boldsymbol{A}=\boldsymbol{U} \boldsymbol{\Sigma} \boldsymbol{V}^{T}$.

9. $\operatorname{diag}(\psi)$ is the diagonal matrix formed from vector $\psi$.

The GMM probability density function for $D$ dimensional point $s$ is:

$$
p(s)=\sum_{i=1}^{M+1} P(i) p(s \mid i)
$$

where the membership probability $P(i)=\frac{1}{M}$, while $p(s \mid i)$ is Gaussian distribution centered on point $\boldsymbol{m}_{i} \in \mathcal{M}$ denoted by:

$$
p(s \mid i)=\frac{1}{\left(2 \pi \sigma^{2}\right)^{D / 2}} \exp \left(-\frac{\left\|s-\boldsymbol{m}_{i}\right\|^{2}}{2 \sigma^{2}}\right)
$$

Therefore, the mixture model function is: 


$$
p(\boldsymbol{s})=w \frac{1}{N}+(1-w) \sum_{i=1}^{M} \frac{1}{M} p(\boldsymbol{s} \mid i)
$$

The GMM centroids are re-parametrized by minimizing the negative log-likelihood function with set of parameters $\theta$, while it is assumed that the data is independent and identically distributed:

$$
E\left(\theta, \sigma^{2}\right)=-\sum_{j=1}^{N} \log \sum_{i=1}^{M+1} P(i) p(s \mid i)
$$

Posterior probability of the GMM centroid $m_{i}$ given the data point $s_{j}$, defines the correspondence probability between these two points:

$$
P\left(i \mid \boldsymbol{s}_{j}\right)=\frac{P(i) p\left(\boldsymbol{s}_{j} \mid i\right)}{p\left(\boldsymbol{s}_{j}\right)}
$$

Parameter $\theta$ and variance $\sigma^{2}$ are found by Expectation Maximization (EM) algorithm, that consists of two steps: first, during the estimation step (or the E-step), it guesses the values of "old" parameters, then it uses Bayes theorem to compute the posterior probability distribution $P^{\text {old }}\left(i \mid \boldsymbol{s}_{j}\right)$ (see Equation A.5). Second, during the maximization step (or M-step), the "new" parameter values are computed by the cost function:

$$
Q\left(\theta, \sigma^{2}\right)=\frac{1}{2 \sigma^{2}} \sum_{j=1}^{N} \sum_{i=1}^{M+1} P^{\mathrm{old}}\left(i \mid \boldsymbol{s}_{j}\right)\left\|\boldsymbol{s}_{j}-\mathcal{T}\left(m_{i} \mid \theta\right)\right\|^{2}+\frac{N_{P} D}{2} \log \sigma^{2}
$$


where

$$
N_{P}=\sum_{j=0}^{N} \sum_{i=0}^{M} P^{\text {old }}\left(i \mid s_{j}\right) \leqslant N
$$

while $N=N_{P}$ only if $w=0$.

Using the previous parameters we can rewrite the posterior probability of GMM components $P^{\text {old }}$ as follows:

$$
P^{\text {old }}\left(j \mid \boldsymbol{s}_{j}\right)=\frac{\exp \left(-\frac{1}{2\left(\sigma^{\text {old }}\right)^{2}}\left\|\boldsymbol{s}_{j}-\mathcal{T}\left(m_{j}, \theta^{\text {old }}\right)\right\|^{2}\right)}{\sum_{k=1}^{M} \exp \left(-\frac{1}{2\left(\sigma^{\text {old }}\right)^{2}}\left\|\boldsymbol{s}_{j}-\mathcal{T}\left(m_{k}, \theta^{\text {old }}\right)\right\|^{2}\right)+\left(2 \pi \sigma^{2}\right)^{\frac{D}{2}} \frac{w}{1-w} \frac{M}{N}}
$$

The Algorithm 6 summarize the steps of non-rigid point set registration. The algorithm aligns point sets for which the transformation assumed to be unknown. 


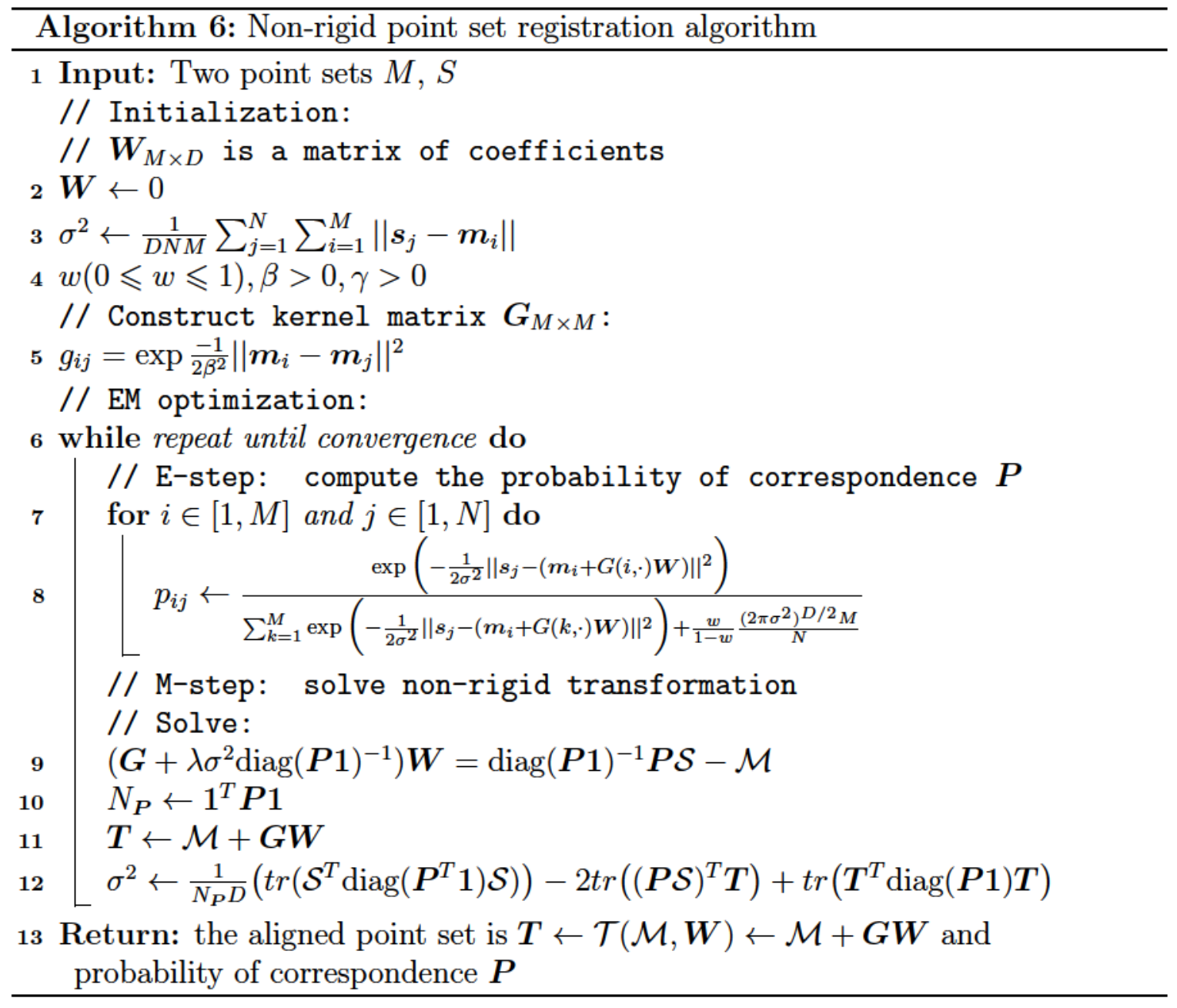




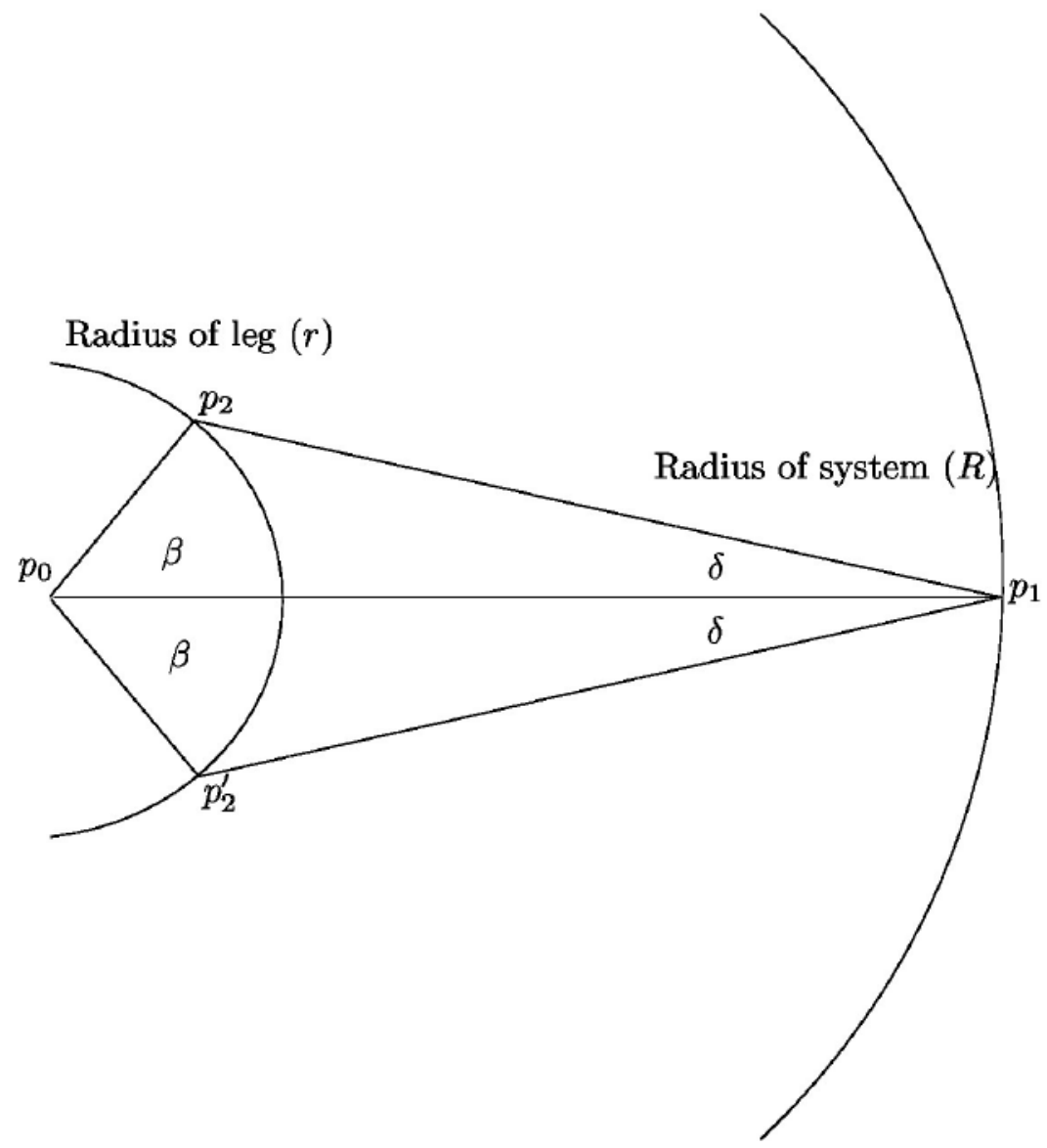

Figure A.1: Sketch of the system.

\section{A.2 Field of View Angle Computation}

The system needs to ensure that each mole appears in at least two images. Thus, the minimum overlap between the two adjacent images is $50 \%$. The overlap amount between two adjacent images during horizontal scan is determined by four parameters: the step size, the view field of the camera $(2 \delta)$, the radius of the leg $(r)$ and the radius of the system $R$.

Figure A.1 presents a sketch of the scanning system with respect to a horizontal scanning. Without loss of generality the figure is presented as a $2 \mathrm{D}$ cross section of 
the scanning system. The centre of the system is at $p_{0}\left(p_{0}=(0,0)\right)$, the camera is positioned along the $\mathrm{x}$-axis at $p_{1}=(R, 0)$ and the leg is at radius $r$. The view angle is $2 \delta$ and the vectors $v=p_{2}-p_{1}$ and $v^{\prime}=p_{2}^{\prime}-p_{1}$ represent the view field of the camera on the leg.

Observation 1 Let $\angle p_{2}^{\prime} p_{1} p_{2}$ be the view angle of the camera and let $\beta=\angle p_{1} p_{0} p_{2}$ be the system step angle. Two consecutive images taken from camera angular position $\gamma$ and $\gamma+\beta$ have an overlap of $50 \%$.

Following Observation 1 the system needs to ensure a step size that guarantees an overlap of at least $50 \%$

Corollary 1 Let $\angle p_{2}^{\prime} p_{1} p_{2}$ be the view angle of the camera and let $\beta=\angle p_{1} p_{0} p_{2}$ be the system step angle. The maximum step size that the system can take between two consecutive images must be smaller than $\beta$.

Since the radius of the leg may change between patients a predefined step size may affect the quality of the images or the amount of time required by the system to complete its scan.

Next we present how to compute $\beta$.

In order to compute $\beta$ the system needs to find $p_{2}$. The point $p_{2}$ is the intersection of the half line starting at $p_{1}$ and has the same direction as $v$. Let $\hat{v}=(-\cos (\delta), \sin (\delta))$ be the unit vector of $v$. Point $p_{2}$ can be computed using the radius, $r$ of the leg.

$$
p_{2}=p_{1}+t * \hat{v}
$$




$$
\begin{aligned}
\left(p_{2}-p_{0}\right)^{2} & =r^{2} \\
\left(p_{1}+t * \hat{v}-p_{0}\right)^{2} & =r^{2} \text { substitute } u=p_{1}-p_{0} \\
(u+t * \hat{v})^{2} & =r^{2} \\
u^{2}+2 u \hat{v} t+\hat{v}^{2} t^{2} & =r^{2} \\
u^{2}+2 u \hat{v} t+\hat{v}^{2} t^{2}-r^{2} & =0 \\
\hat{v}^{2} t^{2}+2 u \hat{v} t+u^{2}-r^{2} & =0 \text { substitute } C=u^{2}-r^{2} \\
\hat{v}^{2} t^{2}+2 u \hat{v} t+C & =0
\end{aligned}
$$

Equation A.16 is a quadratic equation in $t$

$$
\begin{aligned}
t_{1,2} & =\frac{-2 \hat{v} u \mp \sqrt{(2 u \hat{v})^{2}-4 \hat{v}^{2} C}}{2 \hat{v}^{2}} \text { note } \hat{v}^{2}=1 \\
t_{1,2} & =-u \hat{v} \mp \sqrt{(u \hat{v})^{2}-C}
\end{aligned}
$$

A solution to the Equation A.18 exists if $(u \hat{v})^{2}-4 C>=0$. If a solution does not exists then the view angle encompasses all the leg. However, one is interested in determining the step size when the view angle is small.

Given that $p_{0}=(0,0), p_{1}=(R, 0)$ (camera position) and the leg is at radius $r$, The solution $t_{1,2}$ can be determined by 


$$
t_{1,2}=-R(-\cos (\delta)) \mp \sqrt{(-\cos (\delta) R)^{2}-R^{2}+r^{2}}
$$

Once the solution $t_{1,2}$ is found the angle $\beta$ can easily be found by first computing $p_{2}$

$$
\begin{aligned}
& t_{1}=R \cos (\delta)+\sqrt{(-\cos (\delta) R)^{2}-R^{2}+r^{2}} \\
& p_{2}=p_{1}+t_{1} \hat{v}
\end{aligned}
$$

followed by

$$
\beta=\arcsin \left(p_{2} . y / r\right)
$$

where $p_{2} . y$ is the $y$ coordinate of $p_{2}$.

For example $r=10, R=50$ and $\delta=10$

$$
\begin{aligned}
& t_{1}=40 * 0.9848-\sqrt{(-40 * 0.9848)^{2}-40^{2}+10^{2}} \\
& t_{1}=32.1997 \\
& p_{2}=p_{1}+t_{1} \hat{v} \\
& p_{2}=(40,0)+32.1997 *(-0.9848,0.1736) \\
& p_{2}=(8.2897,5.5899)
\end{aligned}
$$


Leading to

$$
\begin{aligned}
\beta & =\arcsin \left(p_{2} . y / r\right) \\
& =\arcsin (5.5899 / 10) \\
& =33.9860
\end{aligned}
$$

